\title{
FORMATION OF RESONANCE DOUBLET PROFILES IN \\ RAPIDLY EXPANDING ENVELOPES
}

\author{
J. SURDEJ* \\ European Southern Observatory, ESO, Geneva, Switzerland
}

(Received 15 April, 1980)

\begin{abstract}
Generalization of the escape probability method introduced by Sobolev allows us to study the transfer of spectral line radiation for a resonance doublet in rapidly expanding envelopes.

For the cases of outward-accelerating (or equivalently inward-decelerating) and outward-decelerating (or equivalently inward-accelerating) envelopes we derive, in the frame of a three-level atom model, the expressions for the spectral radiation fields $J_{12}$ and $J_{13}$, for the resulting radiative force $\mathbf{F}^{R E}$ exerted per atom and for the resonance doublet profile $E(X) / E_{c}$; we take into account the complex radiative coupling, in both resonance transitions $1 \rightleftharpoons 2$ and $1 \rightleftharpoons 3$, between distant parts of the atmosphere.

For various physical and geometrical conditions prevailing in the expanding media. we illustrate and discuss the behaviours of those quantities as well as their dependence on the parameters of the model. Namely, we deduce criteria under which resonance doublet profiles formed in outward-accelerating and/or outward-decelerating envelopes would appear to be resolved into double P Cygni profiles.

We also stress the importance of treating a resonance doublet as being formed by two distinct resonance transitions when evaluating the resulting radiative force $\mathbf{F}^{R E}$ acting on an atom. It is indeed shown that if we use a two-level atom model to represent a resonance doublet - i.e., assigning to it an oscillator strength equal to the sum of the oscillator strengths of both resonance transitions .. the amplitude of the resulting radiative force can be underestimated by factors reaching $100 \%$ and more in the regions of the expanding envelope which are optically thick to the spectral line radiation. In this context, it would be essential to revise the previous models of radiation-driven winds developed for early-type stars in which the lines belonging to any multiplet were treated as a single line.
\end{abstract}

\section{Introduction}

Most of the P Cygni profiles observed in the ultraviolet spectrum of early-type stars and of high redshift quasars are due to the scattering of line photons by a resonance doublet for which the separation of the two upper atomic levels 2 and 3 (see Figure 1), expressed in terms of the Doppler velocity $\Delta v_{23}$, is usually smaller than the maximal expansion velocity $v_{\max }$ of the envelope where they are formed. For instance, we shall mention the resonance doublets $\left({ }^{2} S_{1 / 2}{ }^{-2} P_{1 / 2,3 / 2}^{0}\right)$ of $\mathrm{C}$ IV $(\lambda \lambda 1548.188, \quad 1550.762 \AA), \quad M g$ II $(\lambda \lambda 2795.528, \quad 2802.704 \AA), \quad \mathrm{N} v \quad(\lambda \lambda 1238.808$, $1242.796 \AA), O_{\text {VI }}(\lambda \lambda 1031.928,1037.619 \AA)$ and Si IV $(\lambda \lambda 1393.755,1402.770 \AA)$ for which the separation of the two upper atomic levels amounts to $\Delta v_{23}=498,770$, 965,1653 , and $1939 \mathrm{~km} \mathrm{~s}^{-1}$, respectively. Let us also recall that each of these resonance doublets is characterized by an oscillator strength $f_{13}$ for the line transition $1 \rightleftharpoons 3$ equal to twice the oscillator strength $f_{12}$ for the line transition $1 \rightleftharpoons 2$, and that

* Also, Aspirant au Fonds National de la Recherche Scientifique (Belgium).

Astrophysics and Space Science 73 (1980) 101-158. 0004-640X/80/0731-0101\$08.70

Copyright O 1980 by D. Reidel Publishing Co., Dordrecht, Holland, and Boston, U.S.A. 
the statistical weights of the atomic levels 1,2 and 3 are $g_{1}=2, g_{2}=2, g_{3}=4$, respectively.

In the frame of the Sobolev approximation (Sobolev, 1947, 1958) and using a numerical method of the Monte Carlo type for calculating the transfer of radiation in the resonance transitions $1 \rightleftharpoons 2$ and $1 \rightleftharpoons 3$, Hewitt and Noerdlinger (1974) were the first to present P Cygni profiles formed in outward-accelerating envelopes. Since, however, they neglected to consider the finite size of the stellar core, and, as they did not discuss the behaviour of the source functions, radiative forces, etc., across the media, it is not possible to understand quantitatively the dependence of the calculated P Cygni profiles on the choice of the separation $\Delta v_{23}$ of the two upper atomic levels, of the velocity and opacity distributions, etc.

More recently, Castor and Lamers (1979) have proposed an algorithm for calculating a resonance doublet profile on the basis of the two individual line profiles formed by each of the resonance transitions in an outward-accelerating envelope. Whereas this approach can give satisfactory results under certain physical conditions (for instance, when the fictive opacities $\tau_{12}$ and $\tau_{13}$ are constant across the medium), it remains generally only approximate because the exact description of the line transfer is nots and could not be, included in their simple algorithm.

We give hereafter, also in the context of the Sobolev approximation, a rigorous treatment of the radiative transfer for a resonance doublet in outward-accelerating and outward-decelerating envelopes (later referred to as A.E envelopes and D.E envelopes, respectively).

In Section 2 we establish the form of the statistical equilibrium equations for a three-level atom model (resonance doublet). The expressions of the mean intensities $J_{12}$ and $J_{13}$ in the resonance transitions $1 \rightleftharpoons 2$ and $1 \rightleftharpoons 3$ are presented in Section 3. The expressions for the source functions $S_{12}, S_{13}$, and the radiative forces in A.E and D.E envelopes, as well as some numerical applications and a discussion of these results, are given in Sections 4 and 5, respectively. The formation of resonance doublet profiles in A.E and D.E envelopes is studied and illustrated in Sections 6 and 7 , respectively. Discussion and general conclusions form the last section.

\section{Statistical Equilibrium Equations}

In order to calculate resonance doublet profiles formed in A.E and D.E envelopes we shall adopt an approach similar to the one followed when deriving the expression of the profile for a single resonance transition $1 \rightleftharpoons 2$. Two steps could be distinguished by that method. In the first we solved the statistical equilibrium equation (see Surdej, 1977; referred to below as Paper I) for a two-level atom model in order to evaluate the source function $S_{12}$ and the fictive optical depth $\tau_{12}$ across the atmosphere (Surdej, 1978a - Paper II). The physical conditions having been determined over the whole space of the medium, we could then derive and calculate the expressions of the radiative forces (Surdej, 1978b - Paper III) and of the line profiles formed in A.E and D.E envelopes (Surdej, 1979a - Paper IV). 
Also, for the case of a single resonance transition $1 \rightleftharpoons 2$, Castor and Lamers (1979) have shown that, unless the fictive optical depth $\tau_{12}$ is extremely large, we could always neglect the role of photo-ionizations from the excited atomic level and, consequently, that the transfer of line radiation was perfectly described by a twolevel atom model. Under these same conditions, we use a three-level atom model in order to study the transfer of line radiation for a resonance doublet (see Figure 1).

Assuming that the atomic level populations of a given ion are in a steady state, it is found that the statistical equilibrium equations associated with the atomic levels 2 and 3 have the expressions

$$
n_{1}\left(B_{12} J_{12}+C_{12}\right)=n_{2}\left(B_{21} J_{12}+A_{21}+C_{21}\right)
$$

and

$$
n_{1}\left(B_{13} J_{13}+C_{13}\right)=n_{3}\left(B_{31} J_{13}+A_{31}+C_{31}\right)
$$

where $n_{1}, n_{2}, n_{3}$ represent the volume populations of the levels $1,2,3 ; B_{12}, B_{21}, A_{21}$ and $B_{13}, B_{31}, A_{31}$ the Einstein-Milne transition probabilities for the resonance transitions $1 \rightleftharpoons 2$ and $1 \rightleftharpoons 3 ; C_{12}, C_{21}$ and $C_{13}, C_{31}$ the collisional excitation and de-excitation probabilities; and finally, $J_{12}, J_{13}$ the mean intensities of the spectral radiation fields in the line transitions $1 \rightleftharpoons 2$ and $1 \rightleftharpoons 3$.

Considering the relation between the collisional excitation and de-excitation probabilities $(j=2,3)$,

$$
g_{1} C_{1 j}=g_{j} C_{j 1} \exp \left(-h v_{1 j} / k T_{e}\right),
$$

where $T_{e}$ represents the electronic temperature, and recalling the relations between the Einstein-Milne transition probabilities (cf. relation (6.5) in Paper I), we can easily simplify Equations (1) to the form

$$
S_{12}=\left(1-\varepsilon_{12}\right) J_{12}+\varepsilon_{12} B_{v_{12}}\left(T_{e}\right)
$$

and

$$
S_{13}=\left(1-\varepsilon_{13}\right) J_{13}+\varepsilon_{13} B_{v_{13}}\left(T_{e}\right)
$$

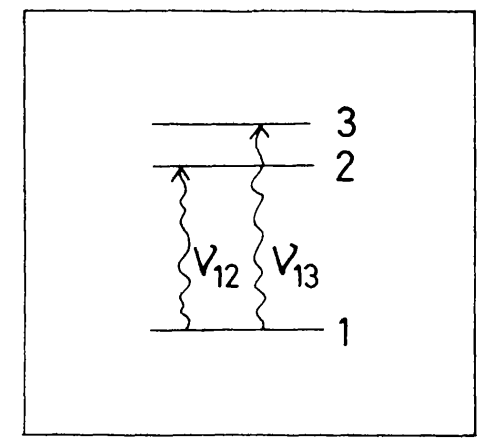

Fig. 1. Lower (1) and upper $(2,3)$ atomic levels corresponding to the radiative transitions $1 \rightleftharpoons 2$ and $1 \rightleftharpoons 3$ for a resonance doublet. 
where the quantity

$$
\varepsilon_{1 j}=\frac{C_{j 1}\left[1-\exp \left(-h v_{1 j} / k T_{e}\right)\right]}{A_{j 1}+C_{j 1}\left[1-\exp \left(-h v_{1 j} / k T_{e}\right)\right]}
$$

represents the probability of de-excitation of the upper level $j$ by collisions (cf. Mihalas, 1970); $B_{v_{12}}\left(T_{e}\right), B_{v_{13}}\left(T_{e}\right)$ the Planck functions calculated at the electronic temperature $T_{e}$ for the transitions $1 \rightleftharpoons 2$ and $1 \rightleftharpoons 3$; and $S_{12}, S_{13}$ the respective source functions expressed in (6.2)-(7.2) of Paper I. When establishing relation (3), we assumed a complete redistribution in frequency and direction for an emitted photon in the frame of a moving atom. Mihalas et al. (1976) showed that this last assumption was a good approximation when calculating the source functions for spectral lines in an expanding medium. It should be noted that it is reasonable to assume that $\varepsilon_{12}=\varepsilon_{13}$ because $C_{21} / A_{21}=\left(C_{31} / A_{31}\right)\left(v_{31} / v_{21}\right)^{3}$ (see Ivanov, 1973).

To avoid further complexities (transfer of ionizing radiation, etc.), we shall not consider here the ionization balance of the atomic species across the medium. Thus, supposing that the amount of matter ejected by the star is constant with time, the volume density $n(r)$,

$$
n(r)=n_{1}+n_{2}+n_{3},
$$

evaluated at a distance $r$ from the centre of the stellar core where the radial velocity is $v(r)$, is given by

$$
n(r)=\frac{n_{0} v_{0}}{v(r)}\left(\frac{1}{L}\right)^{2}
$$

where

$$
L=r / R^{*}
$$

represents the distance $r$ in units of the stellar radius $R^{*}$ and where $n_{0}, v_{0}$ are the volume density and velocity of the matter initially ejected. Let us mention that in order to simulate the P Cygni profiles observed for the resonance doublets of C IV and $\mathrm{Si} \mathrm{IV} \mathrm{in} \mathrm{the} \mathrm{spectrum} \mathrm{of} \mathrm{some} \mathrm{high} \mathrm{redshift} \mathrm{quasars,} \mathrm{we} \mathrm{solved} \mathrm{(Surdej} \mathrm{and}$ Swings, 1980) the same problem when considering that the ionization balance across the envelope is primarily governed by photo-ionization and recombination, the ionizing continuum being optically thin (cf. Castor and Lamers, 1979).

In the next section we deduce the expressions for the mean intensities $J_{12}$ and $J_{13}$ of the spectral radiation fields, on which depend the behaviours of the source functions $S_{12}$ and $S_{13}$ (see relation (3)).

\section{Mean Intensities $J_{12}$ and $J_{13}$ of the Spectral Radiation Fields}

\section{(A) A.E envelopes}

We shall first recall briefly the essence of the Sobolev approximation as well as some important definitions inherent to that formalism. 
The Sobolev approximation (Sobolev, 1947, 1958) consists in assuming that the physical (absorption $\alpha_{i j}$ and emission $\varepsilon_{i j}$ coefficients, electronic temperature $T_{e}$, etc.) and kinematical (velocity gradient $\partial v_{s} / \partial s$, etc.) properties of the medium are homogeneous within spatial intervals $\Delta S$ across which the macroscopic velocity $v_{s}$ of the envelope varies by a quantity $\Delta v_{s}$, roughly equal to the maximal chaotic (thermal and turbulent) velocities $u_{\max }$ of the atoms. Along an axis with abscissae $s$, we have the relation

$$
\Delta S=2 u_{\max } /\left|\frac{\partial v_{s}}{\partial s}\right|,
$$

and provided the velocity gradient $\partial v_{s} / \partial s$ is sufficiently high (rapid expansion), $\Delta S$ will be very small in comparison to the size of the envelope such that the Sobolev approximation formulated above will be fulfilled. In the following, we shall call 'neighbouring' atoms or points, such atoms or points whose relative distance is smaller than $\Delta S$; otherwise, they will be called 'distant'. A 'neighbourhood' around a given point $R$ in the envelope will refer to all points whose distance to $R$ is smaller than $\Delta S$.

It is also very useful to use the concept of 'local frequency' $v_{L}$ of a photon (cf. Magnan, 1973). The local frequency $v_{L}$ of a photon emitted or passing at a given point $R$ is defined as being the frequency seen by an observer moving with the medium at that point. If $v$ denotes the frequency of a line photon, as seen by a fixed observer, we have the first-order Doppler relation

$$
v_{L}=v-v_{i j} \frac{v_{s}}{c}
$$

$v_{s}$ being the macroscopic velocity of the envelope along the direction of propagation of the photon.

A second consequence of the Sobolev approximation is that if at a point $R$ a photon is emitted by an atom with a local frequency $v_{L} \simeq v_{i j}$ in the line transition $i \rightleftharpoons j$ (i.e., $v_{L} \in\left(v_{i j}-\Delta v / 2, v_{i j}+\Delta v / 2\right)$, where $\Delta v$ represents the maximum width of the absorption profile due to chaotic motions of the atoms) it will escape directly from the neighbourhood around $R$ with a probability $\beta_{i j}^{1}$ (see Paper I, Section 2 ) or will scatter any number $n(n=1,2, \ldots)$ of times in that same neighbourhood with a probability $1 / \beta_{i j}^{1}-1$ (see Paper II, Section 3). Because we shall often refer to the escape probability $\beta_{i j}^{1}$, it is useful to recall its expression (see relation (27.2) in Paper I)

$$
\beta_{i j}^{1}=\int_{\Omega=4 \pi} \frac{\left[1-\exp \left(-\tau_{i j}\right)\right]}{\tau_{i j}} \frac{\mathrm{d} \omega}{4 \pi},
$$

where the fictitious opacity

$$
\tau_{i j}=\frac{\alpha_{i j} c}{v_{i j}\left|\partial v_{s} / \partial s\right|}
$$


represents merely the optical depth of the medium in the line transition $i \rightleftharpoons j$ across the distance $\Delta S$ (i.e., $\tau_{i j}=\alpha_{i j} \Delta S / \Delta v$ ).

For a single line transition $i \rightleftharpoons j$, we have shown in Paper I that because of the general state of expansion along all directions in an A.E envelope, the spectral radiation emitted with a local frequency $v_{L} \simeq v_{i j}$ by atoms around a point $R^{\prime}$ could not interact with atoms situated at a distant point $R$ via the same transition. Consequently, we found (cf. Castor, 1970) that the radiation field $J_{i j}$ calculated at a point $R$ was composed of two contributions: a local contribution $J_{i j}^{1}$ due to spectral radiation emitted by the neighbouring atoms and a second, $J_{i j}^{3}$, due to radiation emitted from the stellar core.

When considering a resonance doublet or, more generally speaking, two single line transitions with slightly different rest-frequencies, the situation will be different. Indeed, because of the important Doppler effects across an A.E envelope, we can expect that line radiation emitted with a local frequency $v_{L}^{\prime} \simeq v_{12}$ (resp. $v_{L}^{\prime} \simeq v_{13}$ ) at a point $R^{\prime}$ will interact with distant atoms at a point $R$ via the line transition $1 \rightleftharpoons 3$ (resp. $1 \rightleftharpoons 2$ ). Let us quantitatively investigate these possible effects.

An observer situated at a fixed point $R$ (see Figure 2) and moving with the fluid along the axis $s$ with a velocity $v_{s}$ sees the spectral line radiation emitted by atoms at a distant point $R^{\prime}$ with a local frequency $v_{L}^{\prime} \simeq v_{i j}\left(v_{i j}=v_{12}\right.$ or $\left.v_{i j}=v_{13}\right)$ shifted to the local frequency $v_{L} \simeq v_{k l}\left(v_{k l}=v_{13}\right.$ or $\left.v_{k l}=v_{12}\right)$. By means of relation (9), we easily find the relation between the local frequencies $v_{L}$ and $v_{L}^{\prime}$ as

$$
v_{L}=v_{L}^{\prime}+\frac{v_{i j}}{c}\left(v_{s}^{\prime}-\frac{v_{k l}}{v_{i j}} v_{s}\right),
$$

where $v_{s}^{\prime}$ represents the macroscopic velocity of the fluid at the point $R^{\prime}$ along the axis $s$. Because of the general state of expansion existing in an A.E envelope (see Paper I, Section 3), we have the inequality

$$
v_{s}^{\prime}-v_{s}<0
$$

and since the ratio of frequencies $v_{k l} / v_{i j}$ is of the order of unity, the local frequency $v_{L}$ at $R$ will necessarily be redshifted $\left(v_{L}<v_{L}^{\prime}\right)$ with respect to the initial local frequency $v_{L}^{\prime}$ of the photon emitted at $R^{\prime}$. Therefore, we conclude that in an A.E envelope, only the photons emitted in the transition $3 \rightarrow 1$ of a resonance doublet can interact at distant points with atoms, via the line transition $1 \rightleftharpoons 2$.

Expressing the quantities $v_{s}$ and $v_{s}^{\prime}$ in terms of the radial velocity field $v(r)$ and of the angles $\theta, \theta^{\prime}$ (see Figure 2), we find from relation (12) that the geometrical locus $\left(R, R^{\prime}\right)$ of the distant points $R^{\prime}$ with respect to the fixed point $R$ which satisfies the above condition is given by the set of equations

and

$$
v\left(r^{\prime}\right) \cos \left(\theta^{\prime}\right)=\frac{\lambda_{13}}{\lambda_{12}}\left[v(r) \cos (\theta)+\Delta v_{23}\right]
$$

$$
r^{\prime} \sin \left(\theta^{\prime}\right)=r \sin (\theta)
$$




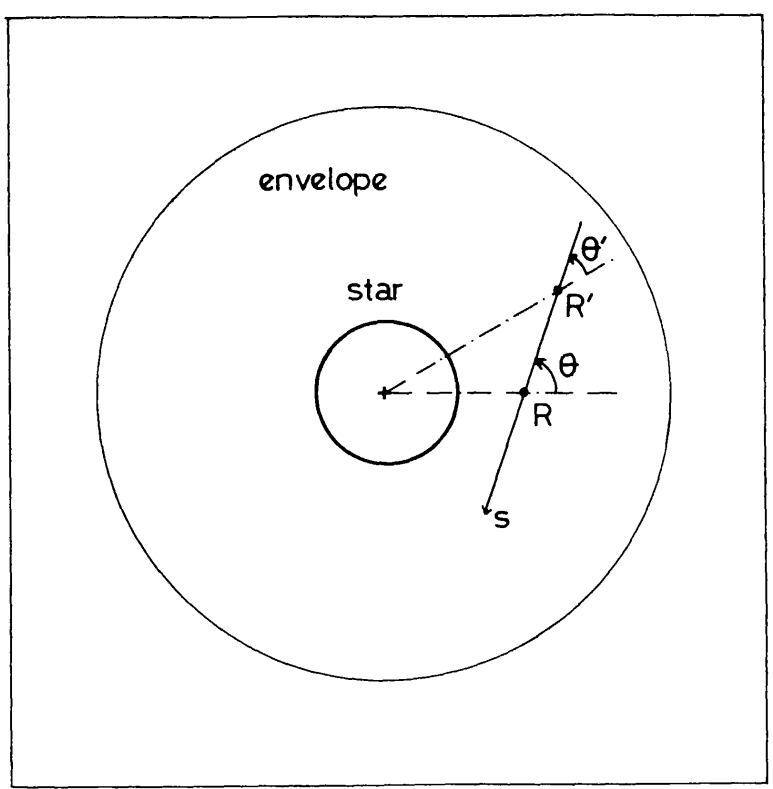

Fig. 2. Arbitrary direction $R^{\prime} R$ in an expanding envelope along which a photon emitted at $R^{\prime}$ with a local frequency $v_{L}^{\prime}$ appears at $R$ with a local frequency $v_{L}$ (see text). The scale is arbitrary.

with

$$
\Delta v_{23}=\frac{\lambda_{12}-\lambda_{13}}{\lambda_{13}} c,
$$

where $\lambda_{12}, \lambda_{13}$ denote the rest-wavelengths for both transitions of the resonance doublet.

The velocity distributions

$$
v(r)=v_{0} L^{-l}
$$

where $v_{0}$ is the velocity at the stellar surface, represent typical A.E envelopes for negative values of the accelerating parameter $l$. Defining by

$$
\Delta X_{23}=\Delta v_{23} / v_{\max }
$$

the ratio of the separation $\Delta v_{23}$ of the two upper atomic levels 2 and 3 (see relation (15)) to the maximal expansion velocity $v_{\max }$ of the envelope, we have illustrated in Figure $3\left(l=-0.5, \Delta X_{23}=0.1\right)$, Figure $4\left(l=-1, \Delta X_{23}=0.1\right)$ and Figure $5(l=-2$, $\left.\Delta X_{23}=0.1\right)$ the geometrical loci $\left(R, R^{\prime}\right)$ of the distant points $R^{\prime}$ with respect to the fixed points $R_{1}(L=1), R_{2}(L=5), R_{3}(L=10)$ and $R_{4}(L=20)$, the maximal extension of the envelope having been arbitrarily chosen to $L_{\max }=20$.

For the value of the accelerating parameter $l=-1$ (see Figure 4), the rate of expansion of the envelope is the same along every direction and at every point $R$, and this explains why the geometrical locus $\left(R, R^{\prime}\right)$ is reduced to a sphere centred on the point $R$ and whose radius $\Delta L$ is given by

$$
\Delta L=\Delta X_{23} L_{\max }
$$




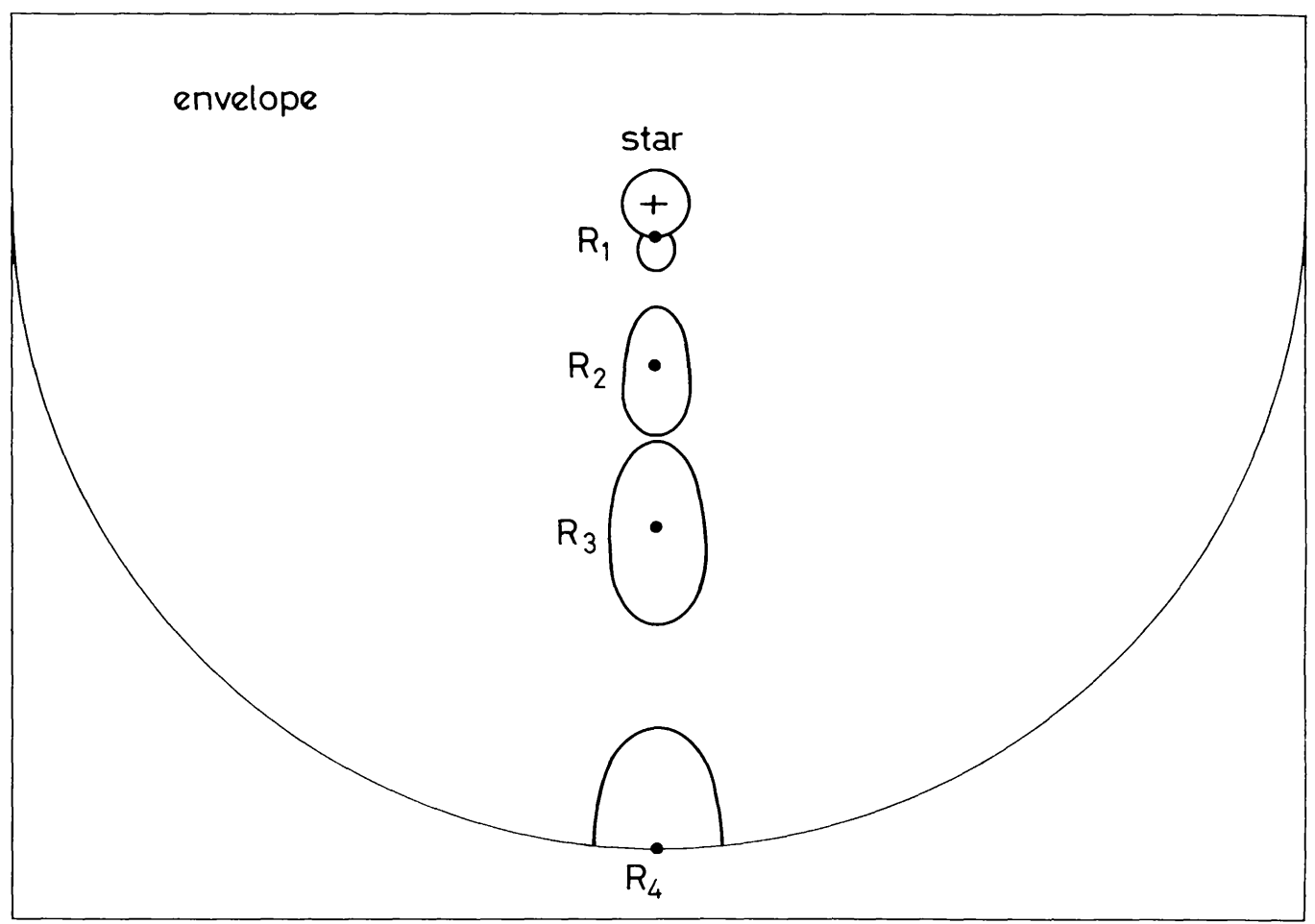

Fig. 3. Geometrical loci $\left(R, R^{\prime}\right)$ with respect to the fixed points $R_{1}(L=1), R_{2}(L=5), R_{3}(L=10)$ and $R_{4}(L=20)$ for the values of the parameters $l=-0.5, \Delta X_{23}=0.1$ and $L_{\max }=20$.

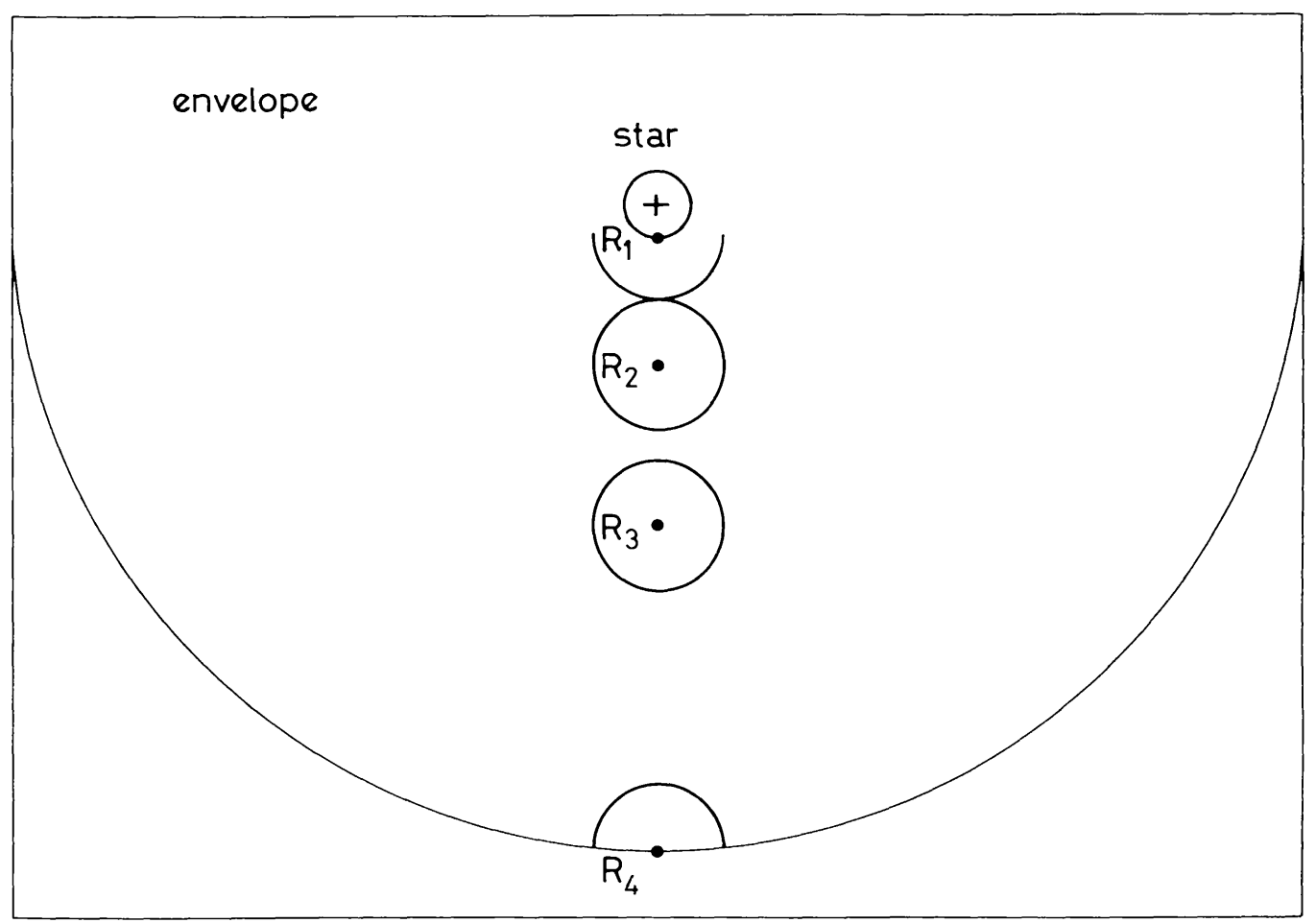

Fig. 4. Geometrical loci $\left(R, R^{\prime}\right)$ with respect to the fixed points $R_{1}(L=1), R_{2}(L=5), R_{3}(L=10)$ and $R_{4}(L=20)$ for the values of the parameters $l=-1, \Delta X_{23}=0.1$ and $L_{\max }=20$. 


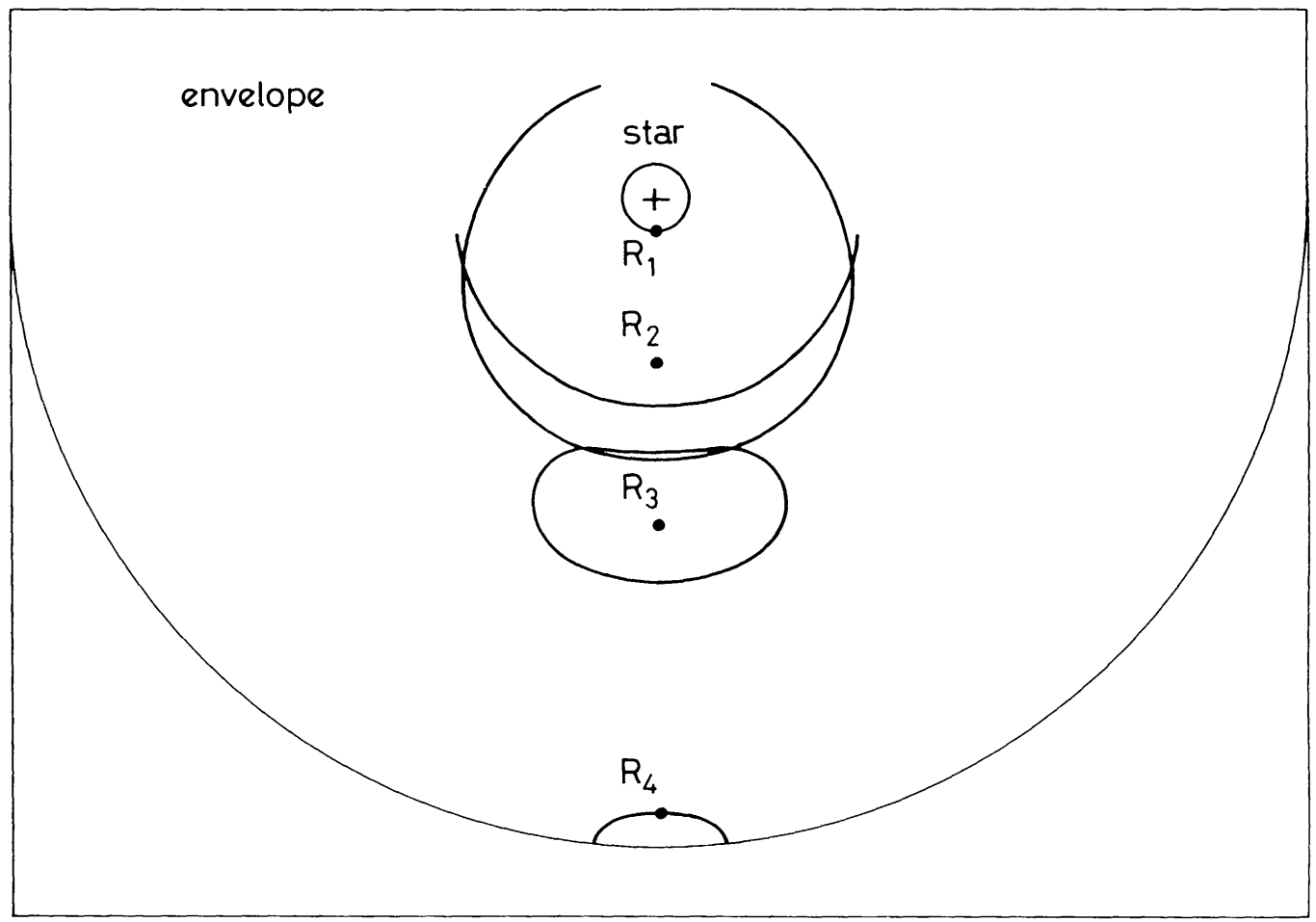

Fig. 5. Geometrical loci $\left(R, R^{\prime}\right)$ with respect to the fixed points $R_{1}(L=1), R_{2}(L=5), R_{3}(L=10)$ and $R_{4}(L=20)$ for the values of the parameters $l=-2, \Delta X_{23}=0.1$ and $L_{\max }=20$.

The results are that if the relative separation $\Delta X_{23}$ of the two upper atomic levels increases, the radiative interactions between atoms situated at points $R$ and $R^{\prime}$ will become more distant and that these will, of course, vanish for $\Delta X_{23} \geqslant 2$.

Inversely, when $\Delta X_{23} \rightarrow 0$-i.e., when the radius $\Delta L$ of the sphere becomes smaller than the distance $\Delta S$ (see relation (8)) across which the macroscopic velocity varies by a quantity $\Delta v_{s}=2 u_{\max }$ - the transfer of spectral line radiation becomes completely local by virtue of the Sobolev approximation. In particular, all calculations (degree of excitation, radiative force, line profile, etc.) performed for the three-level atom model must be identical to those directly obtained via the two-level atom model. Combining relations (8) and (18) for the value of the accelerating parameter $l=-1$, we notice that in practice this will always be the case when

$$
\Delta X_{23}<\frac{2 u_{\max }}{v_{\max }}
$$

We could easily generalize this criterion for an arbitrary velocity field.

For the value of the accelerating parameter $l=-0.5$ (resp. $l=-2$ ) the radial velocity gradient $\mathrm{d} v(r) / \mathrm{d} r$ decreases (resp. increases) outwardly, and by taking into account the angular dependence of the velocity gradient $\partial v_{s} / \partial s$ (cf. relation (40) in Paper II), the different aspects of the geometrical loci illustrated in Figures 3 and 5 are easily understood. 
In order to determine the expressions $J_{12}$ and $J_{13}$ of the spectral radiation fields, we shall first establish the expressions of the fictive opacities $\tau_{12}$ and $\tau_{13}$ (see relation (11)) associated with the resonance transitions $1 \rightleftharpoons 2$ and $1 \rightleftharpoons 3$. Combining the relations (5), (6), (11) and (40), (41) of Paper II for the velocity fields given in (16), we obtain

$$
\tau_{12}=\tau_{12}^{l} L^{2 l-1} F(\theta)\left[1-\left(\frac{g_{1} n_{2}}{g_{2} n_{1}}\right)\right] /\left(1+\frac{n_{2}}{n_{1}}+\frac{n_{3}}{n_{1}}\right)
$$

and

$$
\tau_{13}=\tau_{13}^{l} L^{2 l-1} F(\theta)\left[1-\left(\frac{g_{1} n_{3}}{g_{3} n_{1}}\right)\right] /\left(1+\frac{n_{2}}{n_{1}}+\frac{n_{3}}{n_{1}}\right) .
$$

In these expressions $\tau_{12}^{l}$ and $\tau_{13}^{l}$ represent the radial $(\theta=0)$ fictive opacities $\tau_{12}$ and $\tau_{13}$ at the stellar surface $(L=1)$,

$$
\tau_{12}^{l}=\frac{\pi e^{2}}{m c} f_{12} \lambda_{12} \frac{R^{*} n_{0}}{v_{0}|l|}
$$

and

$$
\tau_{13}^{l}=\frac{\pi e^{2}}{m c} f_{13} \lambda_{13} \frac{R^{*} n_{0}}{v_{0}|l|}
$$

$e$ and $m$ being the charge and mass of the electron, respectively. The second term in Equations (20) expresses the radial dependence of the fictive opacities as a function of $L$. Finally, the function $F(\theta)$ gives the angular correction to be applied to the radial fictive opacities in order to determine $\tau_{12}$ and $\tau_{13}$ along any direction making an angle $\theta$ with the radial direction

$$
F(\theta)=\left|\frac{l}{1-(l+1) \cos ^{2}(\theta)}\right| .
$$

Because there is no perturbation of the radiation field $J_{13}$ by the photons emitted in the resonance transition $1 \rightleftharpoons 2$, its expression is analogous to the one derived for a single line transition $i \rightleftharpoons j$ in an A.E envelope (cf. relation (12) in Paper II),

$$
J_{13}=S_{13}\left(1-\beta_{13}^{1}\right)+I_{c} \beta_{13}^{3} .
$$

We recall that the first term appearing in the right-hand side of this equation represents the local contribution $J_{13}^{1}$ to the mean radiation field $J_{13}$ due to spectral radiation emitted in the transition $1 \rightleftharpoons 3$ by neighboring atoms, and that the second term corresponds to the contribution $J_{13}^{3}$ due to the radiation emitted by the stellar continuum with an intensity $I_{c}$ constant in the frequency interval $v \in\left[v_{12}-v_{12} v_{\max } / c, v_{13}+v_{13} v_{\max } / c\right]$ where the resonance doublet profile is formed. The escape probability $\beta_{13}^{1}$ has been defined in formula (10), and the quantity $\beta_{13}^{3}$ evaluated at a given point $R$ in the envelope (cf. relation (16) in Paper II) represents 
the probability that a photon emitted at the stellar surface will interact with an atom at $R$.

In parallel with the determination of the spectral radiation field in a D.E envelope (see Paper I, Section 4) where we considered, for a single-line transition, the radiative interactions between distant atoms, we can immediately derive the expression for the mean intensity $J_{12}$ of the radiation field in an A.E envelope by taking into account the radiative interactions of atoms at a point $R$ with the field of photons emitted with a local frequency $v_{L}^{\prime} \simeq v_{13}$ by atoms situated along the geometrical locus $\left(R, R^{\prime}\right)$. We easily find that

$$
J_{12}=S_{12}\left(1-\beta_{12}^{1}\right)+I_{c} \beta_{12}^{5}+J_{12}^{2},
$$

where

$$
J_{12}^{2}=\int_{\Omega\left(R, R^{\prime}\right)} S_{13}^{\prime}\left[1-\exp \left(-\tau_{13}^{\prime}\right)\right] \frac{\left[1-\exp \left(-\tau_{12}\right)\right]}{\tau_{12}} \frac{\mathrm{d} \omega}{4 \pi} .
$$

The first term in the right-hand side of Equation (24) accounts for the local contribution $J_{12}^{1}$ to the mean radiation field $J_{12}$. Because the rays subtending the stellar core from a fixed point $R$ in the envelope can intersect the geometrical locus $\left(R, R^{\prime}\right)$ (see Figures 3-5), the second term represents the contribution $J_{12}^{5}$ (cf. relation (27) in Paper II) due to the radiation emitted by the stellar core. The expression for the escape probability $\beta_{12}^{5}$ is straightforward (cf. relation (28) in Paper II): i.e.,

$$
\beta_{12}^{5}=\int_{\Omega=4 \pi W} \frac{\left[1-\exp \left(-\tau_{12}\right)\right]}{\tau_{12}} \exp \left(-\tau_{13}^{\prime}\right) \frac{\mathrm{d} \omega}{4 \pi},
$$

where the factor $\exp \left(-\tau_{13}^{\prime}\right)$ expresses the probability that a photon emitted at the stellar surface towards the point $R$ will not be absorbed in the transition $1 \rightleftharpoons 3$ by atoms situated in the neighbourhood of a distant point $R^{\prime}$. We adopt the convention to set $\tau_{13}^{\prime}=0$ in relation (26) if the direction joining a point of the stellar surface to the point $R$ does not intersect the geometrical locus $\left(R, R^{\prime}\right)$. The geometrical dilution factor $W$ (see relation (10) in Paper II) sets the range of integration of the solid angle $\Omega$ in Equation (26). Finally, the quantity $J_{12}^{2}$ expressed in formula (25) represents the contribution to the mean radiation field $J_{12}$ due to spectral radiation emitted by atoms situated along the geometrical locus $\left(R, R^{\prime}\right)$ with a local frequency $v_{L}^{\prime} \simeq v_{13}$ and appearing at the fixed point $R$ with a local frequency $v_{L} \simeq v_{12}$. The solid angle of integration $\Omega\left(R^{\prime}, R\right)$ covers all directions for which the set of equations (14) does have a solution.

\section{(в) D.E ENVELOPES}

An observer situated at a fixed point $R$ (see Figure 2) in a D.E envelope and moving with the fluid sees, along the radial direction, a general state of contraction of the medium, whereas along a perpendicular axis $\left(\theta=90^{\circ}\right)$ the envelope presents a general state of expansion. Consequently, in addition to a radiative coupling between distant 
atoms emitting and absorbing photons of the same local frequency (see Paper I, Section 4), there exist in a D.E envelope two new types of radiative coupling corresponding to the emission of spectral radiation with a local frequency $v_{L}^{\prime} \simeq v_{13}$ (resp. $\mathrm{v}_{L}^{\prime \prime} \simeq v_{12}$ ) by distant atoms situated at points $R^{\prime}$ (resp. $R^{\prime \prime}$ ) and which can interact with atoms at a point $R$ via the resonance transition $1 \rightleftharpoons 2($ resp. $1 \rightleftharpoons 3$ ).

For the case of D.E envelopes, we shall adopt the velocity fields given in formula (16), where $l(l=0.5,1,2)$ is now a positive parameter expressing a deceleration of the midium around the central core. We shall also consider the velocity fields

$$
v(r)=v_{\infty} \sqrt{1+\frac{G}{L}},
$$

with the decelerating parameter $G$ given by

$$
G=\left(\frac{v_{0}}{v_{\infty}}\right)^{2}-1
$$

which describes the motion of the fluid initially ejected at the stellar surface with a velocity $v_{0}$ and further on decelerated in the gravitational field of the central object. Here $v_{\infty}$ represents the terminal velocity, and we should note that for the case $v_{\infty}=0$, the velocity field given in formula (27) is identical to that in (16) for the value of the decelerating parameter $l=0.5$.

The set of equations (14) allows us to construct immediately the geometrical locus $\left(R, R^{\prime}\right)$ for the above velocity fields. Replacing the frequencies $v_{L}, v_{k l}$ and $v_{L}^{\prime}, v_{i j}$ in relation (12) by $v_{13}$ and $v_{12}$, respectively, we obtain a similar set of equations

$$
v\left(r^{\prime \prime}\right) \cos \left(\theta^{\prime \prime}\right)=\frac{\lambda_{12}}{\lambda_{13}} v(r) \cos (\theta)-\Delta v_{23}
$$

and

$$
r^{\prime \prime} \sin \left(\theta^{\prime \prime}\right)=r \sin (\theta)
$$

which describes the geometrical locus $\left(R, R^{\prime \prime}\right)$ of the distant points $R^{\prime \prime}$ from which spectral radiation emitted with a local frequency $v_{L}^{\prime \prime} \simeq v_{12}$ can interact with atoms situated in the neighbourhood of $R$, via the resonance transition $1 \rightleftharpoons 3$.

For the velocity fields (16) $(l>0)$ and (27), we have illustrated in Figure $6(l=0.5$, $\left.\Delta X_{23}=0.1, L=2\right)$, Figure $7\left(l=1, \Delta X_{23}=0.1, L=1\right)$, Figure $8\left(l=2, \Delta X_{23}=0.1\right.$, $L=2)$ and Figure $9\left(G=10, \Delta X_{23}=0.1, L=2\right)$ the geometrical loci $\left(R, R^{\prime}\right)$ in dashed lines and $\left(R, R^{\prime \prime}\right)$ in dot-dashed lines, the maximal extension of the envelope having been arbitrarily chosen to $L_{\max }=5$. We also recognize in each of these figures the geometrical locus $\left(R, R^{\prime \prime \prime}\right)$ (in full lines) of the distant points $R^{\prime \prime \prime}$ whose relative velocity to the fixed point $R$ is equal to zero (cf. Figure 5 in Paper I).

The four distinct areas separated by the geometrical locus $\left(R, R^{\prime \prime \prime}\right)$, and which are indicated by I, II, III and IV in Figure 6, correspond respectively to the regions of the envelope which present a general state of contraction (I, II), and of expansion 


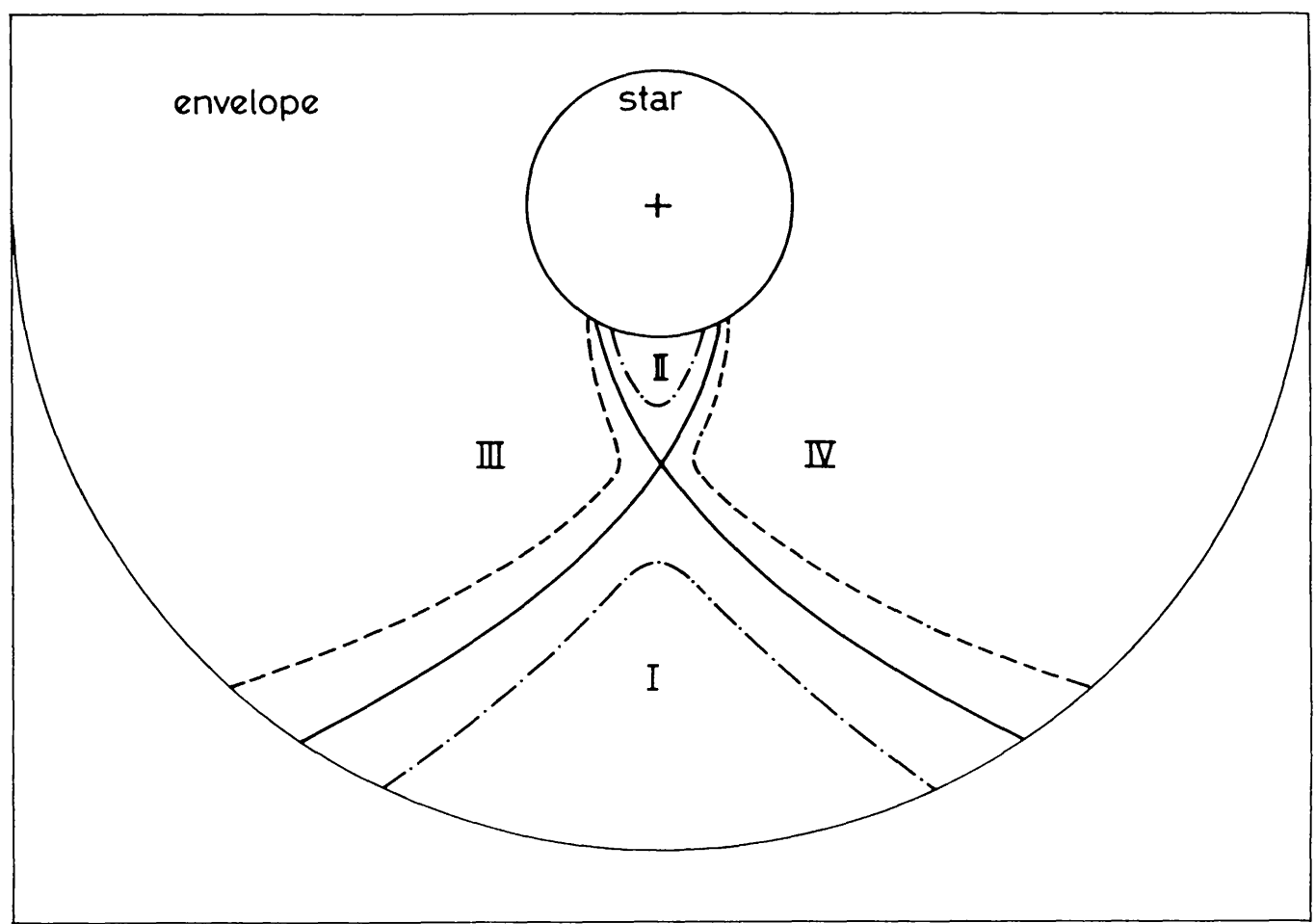

Fig. 6. Geometrical loci $\left(R, R^{\prime}\right)$ (dashed lines), $\left(R, R^{\prime \prime}\right)$ (dot-dashed lines) and $\left(R, R^{\prime \prime \prime}\right)$ (full lines) with respect to the fixed point $R(L=2)$ for the values of the parameters $l=0.5, \Delta X_{23}=0.1$ and $L_{\max }=5$.

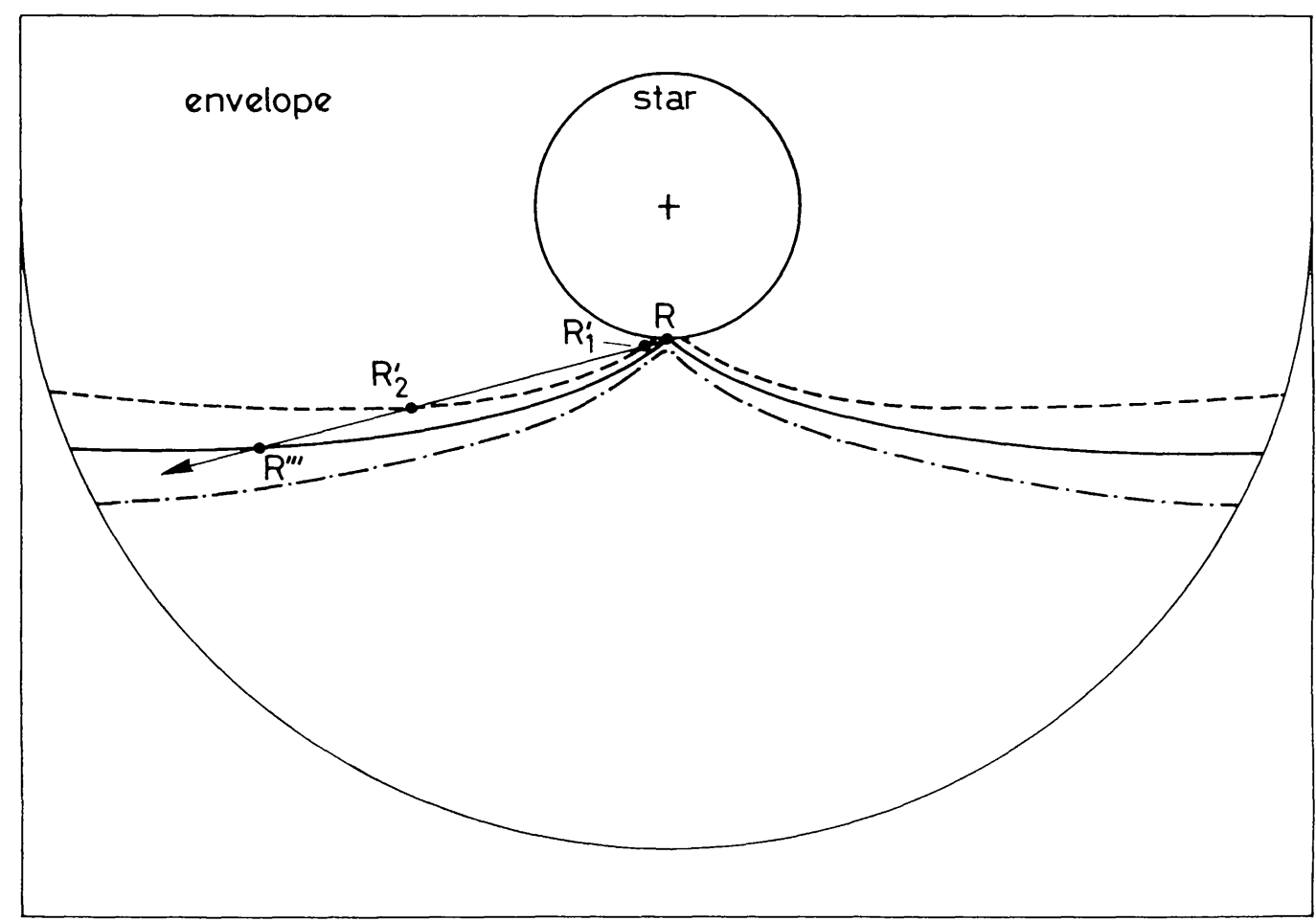

Fig. 7. Geometrical loci $\left(R, R^{\prime}\right)$ (dashed lines), $\left(R, R^{\prime \prime}\right)$ (dot-dashed lines) and $\left(R, R^{\prime \prime \prime}\right)$ (full lines) with respect to the fixed point $R(L=1)$ for the values of the parameters $l=1, \Delta X_{23}=0.1$ and $L_{\max }=5$. 


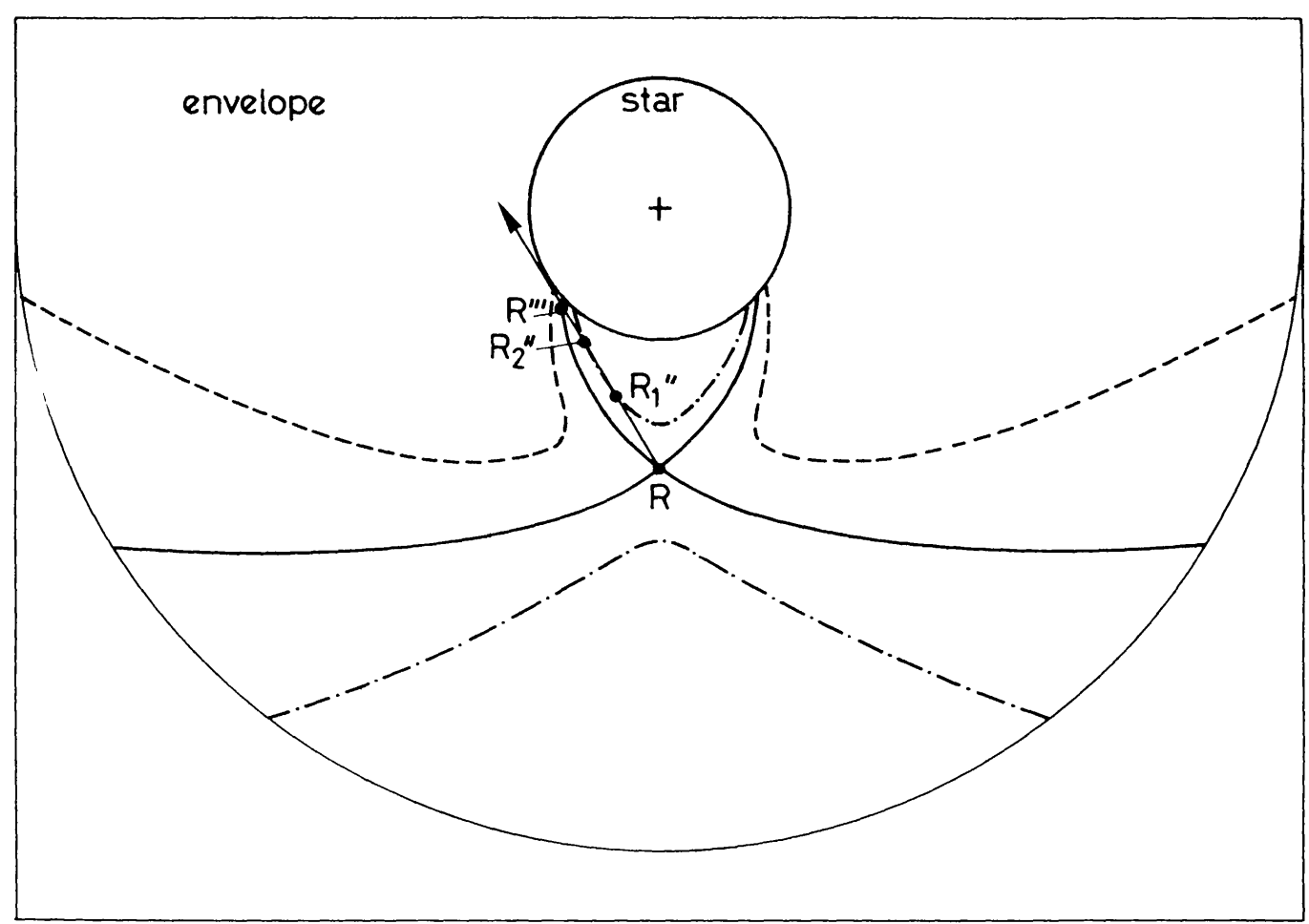

Fig. 8. Geometrical loci $\left(R, R^{\prime}\right)$ (dashed lines), $\left(R, R^{\prime \prime}\right)$ (dot-dashed lines) and $\left(R, R^{\prime \prime \prime}\right)$ (full lines) with respect to the fixed point $R(L=2)$ for the values of the parameters $l=2, \Delta X_{23}=0.1$ and $L_{\max }=5$.

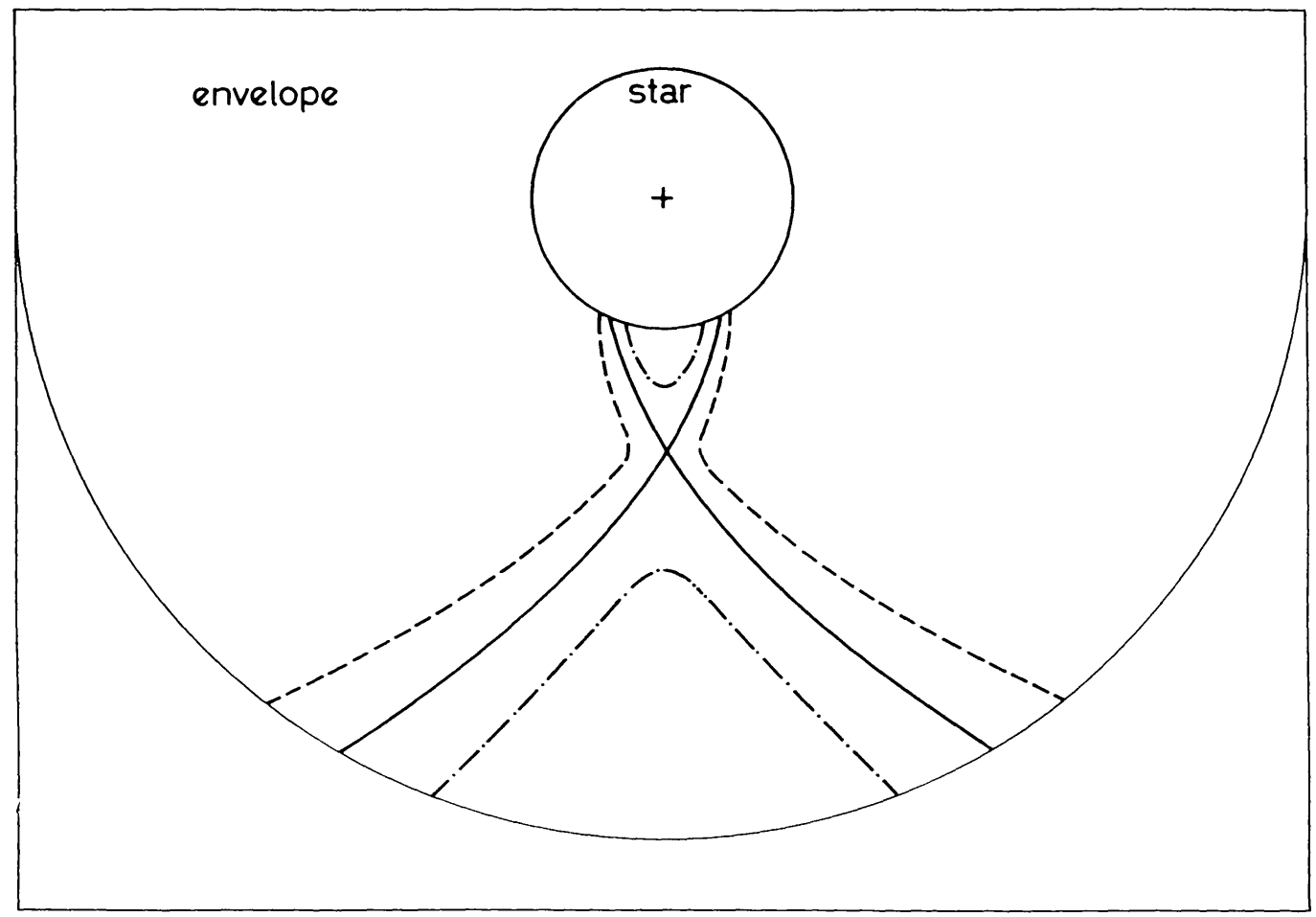

Fig. 9. Geometrical loci $\left(R, R^{\prime}\right)$ (dashed lines), $\left(R, R^{\prime \prime}\right)$ (dot-dashed lines) and $\left(R, R^{\prime \prime \prime}\right)$ (full lines) with respect to the fixed point $R(L=2)$ for the values of the parameters $G=10, \Delta X_{23}=0.1$ and $L_{\max }=5$. 
(III, IV) with respect to the fixed point $R$. With this in mind, we can easily understand the aspect of the geometrical loci $\left(R, R^{\prime}\right)$ and $\left(R, R^{\prime \prime}\right)$ illustrated in Figures 6-9. In particular, when the relative separation $\Delta X_{23}$ of the two upper atomic levels of the resonance doublet gets very small, the geometrical loci $\left(R, R^{\prime}\right)$ and $\left(R, R^{\prime \prime}\right)$ become nearly tangent to the geometrical locus $\left(R, R^{\prime \prime \prime}\right)$ in the respective areas III, IV and I, II. Consequently, when $\Delta X_{23} \rightarrow 0$, the radiative transfer problem for a resonance doublet reduces itself to what we know for a two-level atom model, as we would have expected. Inversely, when $\Delta X_{23}$ increases, the geometrical loci of the distant points $R^{\prime}$ and $R^{\prime \prime}$, whose relative velocity to $R$ is $\Delta v_{s} \simeq \pm \Delta v_{23}$, lie further and further from the geometrical locus $\left(R, R^{\prime \prime \prime}\right)$. Of course, for $\Delta X_{23} \gtrsim 2$, the radiative couplings between distant atoms at points $R, R^{\prime}$ and $R, R^{\prime \prime}$ vanish. Let us also remark that a line of sight directed from $R$ along any direction of space, intersects the geometrical locus $\left(R, R^{\prime}\right)$ (resp. $\left.\left(R, R^{\prime \prime}\right)\right)$ at two distinct points $R_{1}^{\prime}, R_{2}^{\prime}$ (resp. $\left.R_{1}^{\prime \prime}, R_{2}^{\prime \prime}\right)$ at maximum (see Figures 7 and 8). Indeed, for the adopted velocity fields, we have shown in Paper I, Section 4, that along an arbitrary direction in a D.E envelope, two distant points $R, R^{\prime \prime \prime}$ (or $R_{1}^{\prime}, R_{2}^{\prime}$ or $R_{1}^{\prime \prime}, R_{2}^{\prime \prime}$ ) at maximum can have a relative velocity equal to zero.

The expressions of the fictive opacities $\tau_{12}, \tau_{13}$ deduced in (20)-(22) evidently remain identical for the velocity field (16) when $l>0$. Considering the velocity distributions given in (27), it can easily be shown that these expressions take the form

and

$$
\tau_{12}=\tau_{12}^{l} F(L, \theta)\left[1-\left(\frac{g_{1} n_{2}}{g_{2} n_{1}}\right)\right] /\left(1+\frac{n_{2}}{n_{1}}+\frac{n_{3}}{n_{1}}\right)
$$

$$
\tau_{13}=\tau_{13}^{l} F(L, \theta)\left[1-\left(\frac{g_{1} n_{3}}{g_{3} n_{1}}\right)\right] /\left(1+\frac{n_{2}}{n_{1}}+\frac{n_{3}}{n_{1}}\right),
$$

with

$$
F(L, \theta)=1 /\left|2(L / G+1)-(2 L / G+3) \cos ^{2}(\theta)\right|
$$

and where the radial fictive opacities $\tau_{12}^{l}, \tau_{13}^{l}$ at the stellar surface are given by

and

$$
\tau_{12}^{l}=\frac{\pi e^{2}}{m c} f_{12} \lambda_{12} \frac{R^{*} n_{0}}{v_{0}} \frac{2(G+1)}{G}
$$

$$
\tau_{13}^{l}=\frac{\pi e^{2}}{m c} f_{13} \lambda_{13} \frac{R^{*} n_{0}}{v_{0}} \frac{2(G+1)}{G} .
$$

We do now possess all the necessary elements for deriving the expressions $J_{12}$ and $J_{13}$ of the spectral radiation fields in a D.E envelope.

In a general context, let us suppose that the line of sight directed from a fixed point $R$ along any direction of space, intersects the geometrical locus $\left(R, R^{\prime}\right)$ at two distinct points $R_{1}^{\prime}, R_{2}^{\prime}$ (see Figure 7) and the geometrical locus $\left(R, R^{\prime \prime \prime}\right)$ at one point $R^{\prime \prime \prime}$, and that these points $R_{1}^{\prime}, R_{2}^{\prime}$ and $R^{\prime \prime \prime}$ lie, for instance, respectively the nearest 
to $R$. The expression for the mean intensity $J_{12}$ of the spectral radiation field is then straightforward: i.e.,

$$
J_{12}=S_{12}\left(1-\beta_{12}^{1}\right)+I_{c} \beta_{12}^{5}+J_{12}^{2},
$$

where

$$
\begin{aligned}
\beta_{12}^{5}= & \int_{\Omega=4 \pi W} \frac{\left[1-\exp \left(-\tau_{12}\right)\right]}{\tau_{12}} \exp \left[-\tau_{13}\left(R_{1}^{\prime}\right)\right] \times \\
& \times \exp \left[-\tau_{13}\left(R_{2}^{\prime}\right)\right] \exp \left[-\tau_{12}\left(R^{\prime \prime \prime}\right)\right] \frac{\mathrm{d} \omega}{4 \pi}
\end{aligned}
$$

and where

$$
\begin{aligned}
J_{12}^{2}= & \int_{\Omega=4 \pi}\left(S_{12}\left(R^{\prime \prime \prime}\right)\left\{1-\exp \left[-\tau_{12}\left(R^{\prime \prime \prime}\right)\right]\right\} \times\right. \\
& \times \exp \left[-\tau_{13}\left(R_{1}^{\prime}\right)\right] \exp \left[-\tau_{13}\left(R_{2}^{\prime}\right)\right]+ \\
& +S_{13}\left(R_{2}^{\prime}\right)\left\{1-\exp \left[-\tau_{13}\left(R_{2}^{\prime}\right)\right]\right\} \exp \left[-\tau_{13}\left(R_{1}^{\prime}\right)\right]+ \\
& \left.+S_{13}\left(R_{1}^{\prime}\right)\left\{1-\exp \left[-\tau_{13}\left(R_{1}^{\prime}\right)\right]\right\}\right) \frac{\left[1-\exp \left(-\tau_{12}\right)\right]}{\tau_{12}} \frac{\mathrm{d} \omega}{4 \pi}
\end{aligned}
$$

The first term appearing in the right-hand side of relation (33) is the usual local contribution $J_{12}^{1}$ to the radiation field $J_{12}$ due to spectral radiation emitted in the resonance transition $1 \rightleftharpoons 2$ by the neighbouring atoms. The second term accounts for the contribution $J_{12}^{5}$ due to the radiation emitted by the stellar continuum and which has been attenuated along its travel in the neighbourhoods of the points $R_{1}^{\prime}, R_{2}^{\prime}$ and $R^{\prime \prime \prime}$ (see expression (34) for the escape probability $\beta_{12}^{5}$ ). Finally, the last term whose expression is given in (35) contains the different contributions to the mean intensity $J_{12}$ due to spectral line radiation emitted by atoms in the neighbourhoods of the points $R_{1}^{\prime}, R_{2}^{\prime}$ and $R^{\prime \prime \prime}$. For instance, the quantity

$$
S_{12}^{\prime \prime \prime}\left[1-\exp \left(-\tau_{12}^{\prime \prime \prime}\right)\right] \exp \left[-\tau_{13}\left(R_{1}^{\prime}\right)\right] \exp \left[-\tau_{13}\left(R_{2}^{\prime}\right)\right]
$$

represents the spectral intensity radiated at $R^{\prime \prime \prime}$ in the resonance transition $1 \rightleftharpoons 2$ which has been attenuated by atoms in the neighbourhoods of the points $R_{1}^{\prime}, R_{2}^{\prime}$ via absorptions in the resonance transition $1 \rightleftharpoons 3$. The factor $\left[1-\exp \left(-\tau_{12}\right)\right] / \tau_{12}$ evaluated at $R$ represents, of course, the probability that an atom will interact with that spectral radiation at a local frequency $v_{L} \simeq v_{12}$. In expression (35), the integration covers all directions of space $(\Omega=4 \pi)$, with the implicit convention that if the intersection points $R_{1}^{\prime}$ and/or $R_{2}^{\prime}$ and/or $R^{\prime \prime \prime}$ do not exist, the fictive opacities $\tau_{13}\left(R_{1}^{\prime}\right)$ and/or $\tau_{13}\left(R_{2}^{\prime}\right)$ and/or $\tau_{12}\left(R^{\prime \prime \prime}\right)$ are set equal to zero.

Similarly, if we assume that the line of sight directed from a fixed point $R$ along any direction in space intersects the geometrical locus $\left(R, R^{\prime \prime}\right)$ at two distinct points $R_{1}^{\prime \prime}, R_{2}^{\prime \prime}$ (see Figure 8 ) and the geometrical locus $\left(R, R^{\prime \prime \prime}\right)$ at one point $R^{\prime \prime \prime}$, and that 
these points $R_{1}^{\prime \prime}, R_{2}^{\prime \prime}$ and $R^{\prime \prime \prime}$ lie, for instance, respectively the nearest to $R$, the expression for the mean intensity $J_{13}$ of the spectral radiation field is given by

$$
J_{13}=S_{13}\left(1-\beta_{13}^{1}\right)+I_{c} \beta_{13}^{5}+J_{13}^{2},
$$

where

$$
\begin{aligned}
\beta_{13}^{5}= & \int_{\Omega=4 \pi W} \frac{\left[1-\exp \left(-\tau_{13}\right)\right]}{\tau_{13}} \exp \left[-\tau_{12}\left(R_{1}^{\prime \prime}\right)\right] \times \\
& \times \exp \left[-\tau_{12}\left(R_{2}^{\prime \prime}\right)\right] \exp \left[-\tau_{13}\left(R^{\prime \prime \prime}\right)\right] \frac{\mathrm{d} \omega}{4 \pi}
\end{aligned}
$$

and where

$$
\begin{aligned}
J_{13}^{2}= & \int_{\Omega=4 \pi}\left(S_{13}\left(R^{\prime \prime \prime}\right)\left\{1-\exp \left[-\tau_{13}\left(R^{\prime \prime \prime}\right)\right]\right\} \times\right. \\
& \times \exp \left[-\tau_{12}\left(R_{1}^{\prime \prime}\right)\right] \exp \left[-\tau_{12}\left(R_{2}^{\prime \prime}\right)\right]+ \\
& +S_{12}\left(R_{2}^{\prime \prime}\right)\left\{1-\exp \left[-\tau_{12}\left(R_{2}^{\prime \prime}\right)\right]\right\} \exp \left[-\tau_{12}\left(R_{1}^{\prime \prime}\right)\right]+ \\
& \left.+S_{12}\left(R_{1}^{\prime \prime}\right)\left\{1-\exp \left[-\tau_{12}\left(R_{1}^{\prime \prime}\right)\right]\right\}\right) \frac{\left[1-\exp \left(-\tau_{13}\right)\right]}{\tau_{13}} \frac{\mathrm{d} \omega}{4 \pi}
\end{aligned}
$$

The physical meaning of the different terms appearing in expression (36) is analogous to that mentioned above for expression (33).

\section{Source Functions and Radiative Forces for a Resonance Doublet in A.E Envelopes}

(A) Source Functions $S_{12}$ And $S_{13}$

In order to illustrate the behaviour of the source functions $S_{12}$ and $S_{13}$ for a resonance doublet in A.E envelopes, we shall assume that there is conservative scattering in the lines $\left(\varepsilon_{12}=0, \varepsilon_{13}=0\right)$. Castor and Lamers (1979) have discussed this approximation, and have shown that in practice the role of collisional excitation could be neglected provided the lines were not too optically thick.

If we combine Equations (3), (23) and (24), the expressions for the source functions $S_{12}$ and $S_{13}$ take the forms

$$
\begin{aligned}
& S_{12}=I_{c} \beta_{12}^{5} / \beta_{12}^{1}+J_{12}^{2} / \beta_{12}^{1}, \\
& S_{13}=I_{c} \beta_{13}^{3} / \beta_{13}^{1} .
\end{aligned}
$$

We also define the expressions

$$
\begin{aligned}
& L G S_{12}=\log _{10}\left(S_{12}\right), \\
& L G S_{12}^{5}=\log _{10}\left(I_{c} \beta_{12}^{5} / \beta_{12}^{1}\right), \\
& L G S_{12}^{2}=\log _{10}\left(J_{12}^{2} / \beta_{12}^{1}\right), \\
& L G S_{13}=\log _{10}\left(S_{13}\right),
\end{aligned}
$$


which will later be used to illustrate the behaviour of the physical conditions that they represent in A.E envelopes.

\section{(B) RADIATIVE FORCES}

Adopting a similar reasoning to that followed in Paper III when deriving the expression of the radiative force $\mathbf{F}_{i j}$ acting on an atom by absorption of radiation in a single line transition $i \rightleftharpoons j$, it is easy to establish the expression of the resulting radiative force $\mathbf{F}^{R E}$ exerted per atom via the scattering of line photons in the two resonance transitions of a doublet. For the case of an A.E envelope, we find that

$$
\mathbf{F}^{R E}=\mathbf{F}_{12}^{C}+\mathbf{F}_{12}^{A}+\mathbf{F}_{13}^{C},
$$

where the different contributions are expressed by

$$
\begin{aligned}
& \mathbf{F}_{12}^{C}=\frac{4 \pi}{c} k_{12} I_{c} \beta_{12}^{5} \\
& \mathbf{F}_{12}^{A}=\frac{4 \pi}{c} k_{12} \int_{\Omega\left(R, R^{\prime}\right)} S_{13}^{\prime}\left[1-\exp \left(-\tau_{13}^{\prime}\right)\right] \frac{\left[1-\exp \left(-\tau_{12}\right)\right]}{\tau_{12}} \mathbf{l} \frac{\mathrm{d} \omega}{4 \pi}, \\
& \mathbf{F}_{13}^{C}=\frac{4 \pi}{c} k_{13} I_{c} \boldsymbol{\beta}_{13}^{3},
\end{aligned}
$$

$k_{i j}$ being the absorption coefficient per atom for the transition $i \rightleftharpoons j$, and $\mathbf{I}$ a unit vector directed along the incident direction of the photons.

The contribution $\mathbf{F}_{12}^{C}$ (cf. relation (18) in Paper III) represents the radiative force acting on an atom which scatters the stellar radiation via the resonance transition $1 \rightleftharpoons 2$. The escape probability vector $\boldsymbol{\beta}_{12}^{5}$ (cf. relation (20) in Paper III) is here defined by

$$
\boldsymbol{\beta}_{12}^{5}=\int_{\Omega=4 \pi W} \exp \left(-\tau_{13}^{\prime}\right) \frac{\left[1-\exp \left(-\tau_{12}\right)\right]}{\tau_{12}} \mathbf{l} \frac{\mathrm{d} \omega}{4 \pi},
$$

the factor $\exp \left(-\tau_{13}^{\prime}\right)$ accounting for the absorption of the stellar continuum in the neighbourhoods of distant points $R^{\prime}$ (see Figures 3-5) lying between $R$ and the stellar core.

The contribution $\mathbf{F}_{12}^{A}$ (cf. relation (22) in Paper III) evaluated at a point $R$ corresponds to the radiative force exerted per atom, via the transition $1 \rightleftharpoons 2$, by the photons initially emitted with a local frequency $v_{L}^{\prime} \simeq v_{13}$ from the geometrical locus $\left(R, R^{\prime}\right)$.

Finally, the contribution $\mathbf{F}_{13}^{C}$ represents the radiative force acting on an atom which scatters the stellar radiation via the resonance transition $1 \rightleftharpoons 3$. The escape probability vector $\boldsymbol{\beta}_{13}^{3}$ (cf. relation (19) in Paper III) is readily given by

$$
\boldsymbol{\beta}_{13}^{3}=\int_{\Omega=4 \pi W}\left[1-\exp \left(-\tau_{13}\right)\right] / \tau_{13} \mathbf{l} \frac{\mathrm{d} \omega}{4 \pi} .
$$


Noting that all contributions to the resulting radiative force $\mathbf{F}^{R E}$ are directed radially (spherical symmetry!), we choose to define the following quantities

$$
\begin{aligned}
F R E & =\log _{10}\left(\mathbf{F}^{R E} \cdot \mathbf{n} / K\right), \\
F C 12 & =\log _{10}\left(\mathbf{F}_{12}^{C} \cdot \mathbf{n} / K\right), \\
F A 12 & =\log _{10}\left(\left|\mathbf{F}_{12}^{A} \cdot \mathbf{n} / K\right|\right), \\
F C 13 & =\log _{10}\left(\mathbf{F}_{13}^{C} \cdot \mathbf{n} / K\right),
\end{aligned}
$$

in order to illustrate the behaviour of the radiative forces $\mathbf{F}^{R E}, \mathbf{F}_{12}^{C}, \mathbf{F}_{12}^{A}$ and $\mathbf{F}_{13}^{C}$ across an A.E envelope. In these relations, $\mathbf{n}$ is a unit vector directed radially outward and $K$ is a multiplicative constant defined by

$$
K=\frac{4 \pi}{c} I_{c} \frac{\pi e^{2}}{m c}
$$

\section{(C) NumericAl APPLiCATIONS}

The calculation of the radiative forces (see relations (41)-(46)) being very similar to that of the different contributions to the source functions $S_{12}$ and $S_{13}$ (see relation (39)), we have illustrated in Figures 10-13 the behaviour of the quantities $L G S_{12}$, $L G S_{12}^{5}, L G S_{12}^{2}$ and $L G S_{13}$ defined in (40), and in Figures 14-17 that of the quantities $F R E, F C 12, F A 12$ and $F C 13$ defined in (47), as a function of the variable $L$ in A.E envelopes for different geometrical and physical conditions.

When the product $\mathbf{F}_{12}^{A} \cdot \mathbf{n}$ is negative, i.e. the radiative force $\mathbf{F}_{12}^{A}$ is directed radially inward, the corresponding quantity FA12 is represented in Figures 14-17 with dashed lines.

Table I presents a summary of the values for the different physical and geometrical parameters relevant to each of the figures. In this table, column 1 gives the number of the figure which illustrates the behaviour of the physical quantities reported in column 2. Columns 3, 4 and 5 contain, respectively, the values of the parameters $l, \Delta X_{23}$ and $\tau_{12}^{l}$. For all numerical applications, we fixed the maximal extension of

TABLE I

Caption for Figures 10-17

\begin{tabular}{llllc}
\hline Figure & Physical quantities & $l$ & $\Delta X_{23}$ & $\tau_{12}^{l}$ \\
\hline 10 & $L G S_{12}, L G S_{12}^{5}, L G S_{12}^{2}, L G S_{13}$ & -0.5 & 0.25 & 10 \\
11 & $L G S_{12}, L G S_{12}^{5}, L G S_{12}^{2}, L G S_{13}$ & -1 & 0.01 & 100 \\
12 & $L G S_{12}, L G S_{12}^{5}, L G S_{12}^{2}, L G S_{13}$ & -1 & 0.25 & 100 \\
13 & $L G S_{12}, L G S_{12}^{5}, L G S_{12}^{2}, L G S_{13}$ & -2 & 0.25 & $10^{5}$ \\
14 & $F R E, F C 12, F A 12, F C 13, F C A$ & -0.5 & 0.25 & 10 \\
15 & $F R E, F C 12, F A 12, F C 13, F C A$ & -1 & 0.01 & 100 \\
16 & $F R E, F C 12, F A 12, F C 13, F C A$ & -1 & 0.25 & 100 \\
17 & $F R E, F C 12, F A 12, F C 13, F C A$ & -2 & 0.25 & $10^{5}$ \\
\hline
\end{tabular}



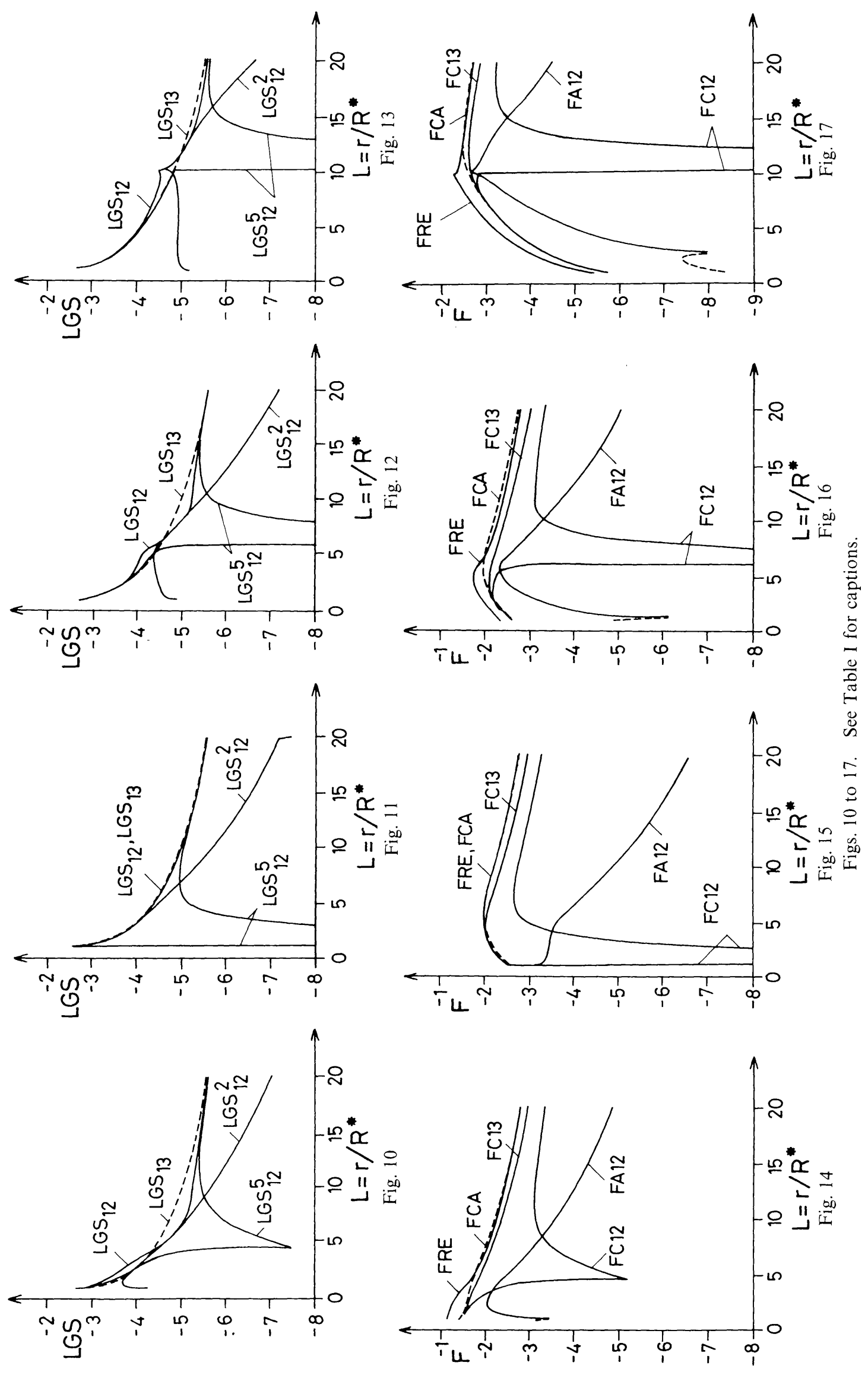
the envelope to $L_{\max }=20$ and we chose the specific intensity $I_{c}$ such that $\lambda_{12} \cdot T=$ $0.28979 \mathrm{~cm}$ deg (Wien's law) with $\lambda_{12}=1.5 \times 10^{-5} \mathrm{~cm}$. Finally, we have chosen the oscillator strengths such that $f_{13}=2 f_{12}$, and thus $\tau_{13}^{l}=2 \tau_{12}^{l}$, and the statistical weights of the atomic levels $g_{1}=2, g_{2}=2$ and $g_{3}=4$ in agreement with the atomic parameters of a typical resonance doublet $\left({ }^{2} S_{1 / 2}{ }^{2} P_{1 / 2,3 / 2}^{0}\right)$.

In order to show the deviations between the resulting radiative force $\mathbf{F}^{R E}$ and the one $\mathbf{F}_{12}^{C A}$ (see relation (17) in Paper III) calculated for a two-level atom model characterized by an oscillator strength $f_{12}^{\prime}=f_{12}+f_{13}$, and thus $\tau_{12}^{l^{\prime}}=3 \tau_{12}^{l}$, and its statistical weights $g_{1}^{\prime}=2, g_{2}^{\prime}=6$, we have also illustrated in Figures 14-17 (see the dashed lines) the corresponding quantity $F C A$ defined by

$$
F C A=\log _{10}\left(\mathbf{F}_{12}^{C A} \cdot \mathbf{n} / K\right) .
$$

\section{(D) Discussion OF THE RESUlts}

Because the mean intensity $J_{13}$ (see relation (23)) of the spectral radiation field is not disturbed by the field of line photons emitted in the resonance transition $1 \rightleftharpoons 2$, the behaviour of the source function $S_{13}$ (see relation (39)) illustrated in Figures 10-13 reduces itself to that given by the two-level atom model (see Paper II). In particular, for the value of the accelerating parameter $l=-1$ (see Figures 11 and 12), we recall that $S_{13}=I_{c} W, W$ being the geometrical dilution factor.

Concerning the source function $S_{12}$, represented by the quantity $L G S_{12}$ in each of the figures, we notice that its behaviour depends critically on those of its contributions $L G S_{12}^{5}$ and $L G S_{12}^{2}$. If we denote $L_{1}$ the distance between the centre of the stellar core and the point $R$ at which the stellar disk appears completely occulted by the geometrical locus $\left(R, R^{\prime}\right)$ and since $\tau_{13}>1$ at $L \gtrsim 1$, we can easily understand the behaviour of the quantity $L G S_{12}^{5}$ which suddenly decreases for $L \gtrsim L_{1}$ (see Figures 10-13). For the values $\theta=\pi, \theta^{\prime}=\pi$ and $L^{\prime}=1$, we deduce from relation (14)

$$
L_{1} \simeq\left(1+\Delta X_{23} L_{\max }^{-l}\right)^{-1 / l} .
$$

Further out, the contribution $L G S_{12}^{5}$ increases appreciably and even becomes superior to $L G S_{12}^{2}$ at a certain distance that we shall denote $L_{2}$. Indeed, when the distance $L$ increases, the fictive opacity $\tau_{13}^{\prime}$, evaluated at the intersection between the geometrical locus $\left(R, R^{\prime}\right)$ and the radial direction, decreases (see relations (14) and (20)) and, consequently, the attenuation factor exp $\left(-\tau_{13}^{\prime}\right)$ becoming greater in relations (25) and (26), the different behaviours of the quantities $L G S_{12}^{5}$ and $L G S_{12}^{2}$ are simply interpreted. Replacing $\tau_{13}^{\prime}=1$ in relation (20) and $\theta=\pi, \theta^{\prime}=\pi$ in relation (14), we obtain

$$
L_{2} \simeq\left[\tau_{13}^{l(l / 2 l-1)}+\Delta X_{23} L_{\max }^{-l}\right]^{-1 / l}
$$

Representing by $\left[L_{1}, L_{2}\right]$ the approximate interval in which the source function $L G S_{12}$ is dominated by $L G S_{12}^{2}$, we find by means of relations (50) and (51) for the respective Figures $10-13$ that it must be so for $L \in[4.5,10.5],[1.2,6.0],[6.0,10.8]$ 
and $[10.0,15.2]$. These results are in good agreement with those illustrated in the corresponding figures.

The behaviour of $L G S_{12}^{2}$, which increases as far as $L=L_{1}$ and decreases further out, can be understood on the basis of the aspect of the geometrical locus $\left(R, R^{\prime}\right)$ (see Figures 3-5) and of the distributions for the source function $S_{13}$ (see Figures 10-13) and for the fictive opacity $\tau_{13}$ (see relation (20)). For instance, for the particular case $l=-1$, the geometrical locus of the distant points $R^{\prime}$, first reduced to half a sphere at $L=1$, gets developed into a complete sphere at $L=L_{1}$. It results from this that in the interval $L \in\left[1, L_{1}\right]$ the contribution $L G S_{12}^{2}$ is an increasing function of $L$. For $L$ increasing over $L_{1}$, both the source function $S_{13}$ and the fictive opacity $\tau_{13}$ evaluated over the sphere $\left(R, R^{\prime}\right)$ decrease and so does the contribution $L G S_{12}^{2}$.

Recalling that when $\Delta X_{23} \rightarrow 0$ (see Figure 11, $\Delta X_{23}=0.01$ ), the three-level atom model must account for the results directly obtained by means of the two-level atom model, the following relation must hold

$$
g_{2}^{\prime} S_{12}^{\prime}=g_{2} S_{12}+g_{3} S_{13},
$$

where $S_{12}^{\prime}$ represents the source function for the two-level atom model and $g_{2}^{\prime}$ the statistical weight of its upper level. This condition is fulfilled for the results in Figure 11. Indeed, for $l=-1$, we have $S_{12}^{\prime}=S_{13}=I_{c} W$ and this implies $S_{12}=S_{13}$.

In conclusion, we see that for a three-level atom (resonance doublet) embedded in an A.E envelope, it is important to take into account the radiative interactions between distant atoms in order to derive the exact population of the first upper atomic level.

In the rest of this paragraph we shall discuss the behaviour of the radiative forces $\mathbf{F}^{R E}, \mathbf{F}_{12}^{C}, \mathbf{F}_{12}^{A}, \mathbf{F}_{13}^{C}$ and $\mathbf{F}_{12}^{C A}$, illustrated, respectively, by the quantities $F R E, F C 12$, $F A 12, F C 13$ and $F C A$ in Figures 14-17.

Because we have shown that in an A.E envelope all radiative processes taking place in the resonance transition $1 \rightleftharpoons 3$ could be interpreted on the basis of the two-level atom model, we refer the reader to Paper III for the discussion of the behaviour of the quantity $F C 13$, and also for that of $F C A$.

The analogy existing respectively between the expressions of the quantities $J_{12}^{5}$, $J_{12}^{2}$ (see relations (24)-(26)) and $\mathbf{F}_{12}^{C}, \mathbf{F}_{12}^{A}$ (see relations (42)-(43) and (45)) implies that, to some extent, the behaviour of the contributions FC12 and FA12 to the radiative force $\mathbf{F}^{R E}$ acting on an atom via the resonance transition $1 \rightleftharpoons 2$ is similar to those of the contributions $L G S_{12}^{5}$ and $L G S_{12}^{2}$ discussed above. In particular, for $L<L_{1}$ or $L>L_{2}$ we do observe $F C 12>F A 12$, whereas in the interval $L \in\left[L_{1}, L_{2}\right]$ we have the reverse. Let us also remark that near the stellar core the radiative force $\mathbf{F}_{12}^{A}$ is directed radially inward, but remains in amplitude much smaller than the contribution $\mathbf{F}_{12}^{C}$ due to the stellar continuum. This behaviour bears a strong resemblance to that of the radiative force $\mathbf{F}_{i j}^{D D}$ (see relation (22) in Paper III) for a single line transition $i \rightleftharpoons j$, due to the radiative interactions between distant atoms in a D.E envelope. Indeed, noticing that at the 
stellar surface we have $\mathbf{l} \cdot \mathbf{n} \leqslant 0$ for all directions intersecting the geometrical locus $\left(R, R^{\prime}\right)$, we easily understand that near the core, the resulting impulsion carried by the photons emitted with a local frequency $v_{L}^{\prime} \simeq v_{13}$ from the geometrical locus $\left(R, R^{\prime}\right)$ will be directed radially inward.

As expected, when the relative separation $\Delta X_{23}$ of the upper atomic levels gets very small (see Figure $15, \Delta X_{23}=0.01$ ), the quantity $F R E$ corresponding to the resulting radiative force $\mathbf{F}^{R E}$ becomes nearly equal to the quantity $F C A$, calculated for the two-level atom model $\left(\tau_{12}^{\prime l}=3 \tau_{12}^{l}, g_{1}^{\prime}=2, g_{2}^{\prime}=6\right)$.

It is very interesting to notice in Figures 14-17 that the quantities $F C A$ and/or $F C 13$ and/or $F C 12$ are represented by the same curve in the regions of the envelope where the respective conditions $\tau_{12}^{\prime}=\tau_{12}+\tau_{13}>1$ and/or $\tau_{13}>1$ and/or $\tau_{12}>1, L<L_{1}$ are fulfilled. These behaviours are simply interpreted if we remember (cf. Lucy, 1971; Castor, 1974, and Paper III, Section 4) that the radiative force $\mathbf{F}_{i j}$ associated with a single line transition $i \rightleftharpoons j$ is independent of the oscillator strength $f_{i j}$ when the medium is optically thick to the spectral line radiation $\left(\tau_{i j}>1\right)$. Indeed, when $L<L_{1}$ and $\tau_{12}>1$, the expressions (17) of Paper III and (42)-(44) for the radiative forces $\mathbf{F}_{12}^{C A}, \mathbf{F}_{12}^{C}$ and $\mathbf{F}_{13}^{C}$ can be rewritten in the form

$$
\begin{aligned}
& \mathbf{F}_{12}^{C A} \simeq \frac{4 \pi}{c} \frac{I_{c}}{n_{1}} \frac{v_{12}^{\prime}}{c} \int_{\Omega=4 \pi W}\left|\frac{\partial v_{s}}{\partial s}\right| \mathbf{I} \frac{\mathrm{d} \omega}{4 \pi} \\
& \mathbf{F}_{12}^{C} \simeq \frac{4 \pi}{c} \frac{I_{c}}{n_{1}} \frac{v_{12}}{c} \int_{\Omega=4 \pi W}\left|\frac{\partial v_{s}}{\partial s}\right| \mathbf{I} \frac{\mathrm{d} \omega}{4 \pi} \\
& \mathbf{F}_{13}^{C} \simeq \frac{4 \pi}{c} \frac{I_{c}}{n_{1}} \frac{v_{13}}{c} \int_{\Omega=4 \pi W}\left|\frac{\partial v_{s}}{\partial s}\right| \mathbf{I} \frac{\mathrm{d} \omega}{4 \pi}
\end{aligned}
$$

and since $v_{12}^{\prime} \simeq v_{12} \simeq v_{13}$, we find with a good approximation that

$$
\mathbf{F}_{12}^{C A} \simeq \mathbf{F}_{12}^{C} \simeq \mathbf{F}_{13}^{C} \text {. }
$$

Consequently, in the region $L<L_{1}$ of an A.E envelope where the condition $\tau_{12}>1$ is fulfilled, the radiative force $\mathbf{F}^{R E}$ resulting from the single contributions $\mathbf{F}_{12}^{C}, \mathbf{F}_{12}^{A}$ and $\mathbf{F}_{13}^{C}$ (see Figures 14-17) appears at least twice higher than the force $\mathbf{F}_{12}^{C A}$ calculated by supposing that the components of a resonance doublet form a single line transition.

Lucy and Solomon $(1967,1970)$ and Castor et al. (1975) have proposed an ejection mechanism in order to explain the origin of the mass-loss observed in a great number of early-type stars. Though Castor et al. have refined the hydrodynamical model of Lucy and Solomon by considering that all line transitions $i \rightleftharpoons j$ of the most abundant ions contributed to the radiative force acting on the gas, they nevertheless assumed that the set of lines belonging to a same multiplet (resonance doublet, etc.) formed a single line transition. Because in most parts of the envelopes they considered, the medium is optically thick to the spectral line radiation $\left(\tau_{i j}>1\right)$, the fact they did 
not take into account the structure of the multiplets in the calculation of the resulting radiative force implies that they underestimated the latter one (at least in the region near the star where the flow is the most accelerated!) and thus also the rates of mass-loss they derived. It would be essential to treat again the same hydrodynamical problem by including the structure of each multiplet.

Let us finally notice that in the region of the envelope where the conditions $\tau_{13}<1, \tau_{12}^{\prime}>1$ are fulfilled (see Figures 14-17), the resulting radiative force $\mathbf{F}^{R E}$ appears slightly smaller than the radiative force $\mathbf{F}_{12}^{C A}$, but as soon as $\tau_{12}^{\prime}<1$ and $L>L_{2}$ we have

with

$$
\mathbf{F}_{12}^{A} \ll \mathbf{F}_{12}^{C}
$$

$$
\mathbf{F}_{12}^{C} \simeq \frac{4 \pi}{c} I_{c} k_{12} / L^{2} \cdot \mathbf{n}
$$

and

$$
\mathbf{F}_{13}^{C} \simeq \frac{4 \pi}{c} I_{c} k_{13} / L^{2} \cdot \mathbf{n}
$$

consequently

$$
\mathbf{F}^{R E} \simeq \frac{4 \pi}{c} I_{c}\left(k_{12}+k_{13}\right) / L^{2} \cdot \mathbf{n}
$$

just as

$$
\mathbf{F}_{12}^{C A} \simeq \frac{4 \pi}{c} I_{c} k_{12}^{\prime} / L^{2} \cdot \mathbf{n},
$$

where the absorption coefficient $k_{12}^{\prime}$ per atom is given by

$$
k_{12}^{\prime}=k_{12}+k_{13} \text {. }
$$

Sufficiently far from the stellar core $\left(\tau_{12}^{\prime}<1, L>L_{2}\right)$, the resulting radiative force $\mathbf{F}^{R E}$ is inversely proportional to the square of the distance $L$ (geometrical dilution) and is perfectly represented by the radiative force $\mathbf{F}_{12}^{C A}$ calculated for the two-level atom model.

We conclude that it is very important to take into account the structure of a resonance doublet (and a fortiori of any multiplet!) in order to derive the exact behaviour and amplitude of the resulting radiative force $\mathbf{F}^{R E}$ acting on atoms in an A.E envelope. While in the distant regions of the envelope where the condition $\tau_{12}^{\prime}<1$ is fulfilled, the radiative force $\mathbf{F}_{12}^{C A}$ calculated by supposing that the doublet is represented by a single line transition gives a correct description of the behaviour of the resulting radiative force $\mathbf{F}^{R E}$, this approximation is no longer valid in the regions near the stellar core where the medium is generally optically thick $\left(\tau_{12}>1\right)$ to the spectral line radiation. We have shown that in this case the resulting radiative force $\mathbf{F}^{R E}$ calculated for a resonance doublet appeared at least twice higher than the single radiative force $\mathbf{F}_{12}^{C A}$ and that this could affect appreciably the determination of mass-loss rates. 


\section{Source Functions and Radiative Forces for a Resonance Doublet in D.E Envelopes}

(A) Source Functions $S_{12}$ And $S_{13}$

As previously, we shall assume that there is conservative scattering in the lines $\left(\varepsilon_{12}=0, \varepsilon_{13}=0\right)$. If we combine Equations (3), (33) and (36), the expressions for the source functions $S_{12}$ and $S_{13}$ in a D.E envelope take the forms

$$
\begin{aligned}
& S_{12}=I_{c} \beta_{12}^{5} / \beta_{12}^{1}+J_{12}^{2} / \beta_{12}^{1}, \\
& S_{13}=I_{c} \beta_{13}^{5} / \beta_{13}^{1}+\mathbf{J}_{13}^{2} / \beta_{13}^{1} .
\end{aligned}
$$

The following expressions are defined

$$
\begin{aligned}
& L G S_{12}=\log _{10}\left(S_{12}\right), \\
& L G S_{12}^{5}=\log _{10}\left(I_{c} \beta_{12}^{5} / \beta_{12}^{1}\right), \\
& L G S_{12}^{2}=\log _{10}\left(J_{12}^{2} / \beta_{12}^{1}\right), \\
& L G S_{13}=\log _{10}\left(S_{13}\right), \\
& L G S_{13}^{5}=\log _{10}\left(I_{c} \beta_{13}^{5} / \beta_{13}^{1}\right), \\
& L G S_{13}^{2}=\log _{10}\left(J_{13}^{2} / \beta_{13}^{1}\right),
\end{aligned}
$$

and will be later used to illustrate the behaviour of the physical conditions they represent in D.E envelopes.

\section{(B) RADiATIVE FORCES}

On the basis of the results derived in Section 2 of Paper III and in Sections 3(B) and 4(B), we easily find that the expression of the resulting radiative force $\mathbf{F}^{R E}$ acting on an atom via the resonance transitions $1 \rightleftharpoons 2$ and $1 \rightleftharpoons 3$ in a D.E envelope is given by

$$
\mathbf{F}^{R E}=\mathbf{F}_{12}^{C}+\mathbf{F}_{12}^{D}+\mathbf{F}_{13}^{C}+\mathbf{F}_{13}^{D},
$$

where the different contributions are, respectively, expressed by

$$
\begin{aligned}
\mathbf{F}_{12}^{C}= & \frac{4 \pi}{c} k_{12} I_{c} \boldsymbol{\beta}_{12}^{5}, \\
\mathbf{F}_{12}^{D}= & \frac{4 \pi}{c} k_{12} \int_{\Omega=4 \pi}\left(S_{12}\left(R^{\prime \prime \prime}\right)\left\{1-\exp \left[-\tau_{12}\left(R^{\prime \prime \prime}\right)\right]\right\} \times\right. \\
& \times \exp \left[-\tau_{13}\left(R_{1}^{\prime}\right)\right] \exp \left[-\tau_{13}\left(R_{2}^{\prime}\right)\right]+ \\
& +S_{13}\left(R_{2}^{\prime}\right)\left\{1-\exp \left[-\tau_{13}\left(R_{2}^{\prime}\right)\right]\right\} \exp \left[-\tau_{13}\left(R_{1}^{\prime}\right)\right]+ \\
& \left.+S_{13}\left(R_{1}^{\prime}\right)\left\{1-\exp \left[-\tau_{13}\left(R_{1}^{\prime}\right)\right]\right\}\right) \frac{\left[1-\exp \left(-\tau_{12}\right)\right]}{\tau_{12}} \mathbf{l} \frac{\mathrm{d} \omega}{4 \pi},
\end{aligned}
$$




$$
\mathbf{F}_{13}^{C}=\frac{4 \pi}{c} k_{13} I_{c} \boldsymbol{\beta}_{13}^{5}
$$

and

$$
\begin{aligned}
\mathbf{F}_{13}^{D}= & \frac{4 \pi}{c} k_{13} \int_{\Omega=4 \pi}\left(S_{13}\left(R^{\prime \prime \prime}\right)\left\{1-\exp \left[-\tau_{13}\left(R^{\prime \prime \prime}\right)\right]\right\} \times\right. \\
& \times \exp \left[-\tau_{12}\left(R_{1}^{\prime \prime}\right)\right] \exp \left[-\tau_{12}\left(R_{2}^{\prime \prime}\right)\right]+ \\
& +S_{12}\left(R_{2}^{\prime \prime}\right)\left\{1-\exp \left[-\tau_{12}\left(R_{2}^{\prime \prime}\right)\right]\right\} \exp \left[-\tau_{12}\left(R_{1}^{\prime \prime}\right)\right]+ \\
& \left.+S_{12}\left(R_{1}^{\prime \prime}\right)\left\{1-\exp \left[-\tau_{12}\left(R_{1}^{\prime \prime}\right)\right]\right\}\right) \frac{\left[1-\exp \left(-\tau_{13}\right)\right]}{\tau_{13}} \mathbf{I} \frac{\mathrm{d} \omega}{4 \pi} .
\end{aligned}
$$

In deriving these expressions we adopted the same conventions as in Section 3(B) namely that the line of sight directed from a fixed point $R$ along any direction of space intersects the geometrical locus $\left(R, R^{\prime}\right)$ (resp. $\left(R, R^{\prime \prime}\right)$ ) at two distinct points $R_{1}^{\prime}, R_{2}^{\prime}$ (resp. $\left.R_{1}^{\prime \prime}, R_{2}^{\prime \prime}\right)$ and the geometrical locus $\left(R, R^{\prime \prime \prime}\right)$ at one point $R^{\prime \prime \prime}$ and that these points $R_{1}^{\prime}, R_{2}^{\prime}, R^{\prime \prime \prime}$ (resp. $R_{1}^{\prime \prime}, R_{2}^{\prime \prime}, R^{\prime \prime \prime}$ ) do lie respectively the nearest to the point $R$ (see Figures 7 and 8 ).

The contributions $\mathbf{F}_{12}^{C}$ and $\mathbf{F}_{13}^{C}$, expressed in (62) and (64), represent the radiative forces acting on an atom which scatters the radiation emitted from the stellar core, respectively via the resonance transitions $1 \rightleftharpoons 2$ and $1 \rightleftharpoons 3$. The escape probability vectors $\boldsymbol{\beta}_{12}^{5}$ and $\boldsymbol{\beta}_{13}^{5}$ which appear in the corresponding expressions are given (cf. relations (34) and (37)) by

$$
\begin{aligned}
\boldsymbol{\beta}_{12}^{5}= & \int_{\Omega=4 \pi W} \frac{\left[1-\exp \left(-\tau_{12}\right)\right]}{\tau_{12}} \exp \left[-\tau_{13}\left(R_{1}^{\prime}\right)\right] \times \\
& \times \exp \left[-\tau_{13}\left(R_{2}^{\prime}\right)\right] \exp \left[-\tau_{13}\left(R^{\prime \prime \prime}\right)\right] \mathrm{l} \frac{\mathrm{d} \omega}{4 \pi}
\end{aligned}
$$

and

$$
\begin{aligned}
\boldsymbol{\beta}_{13}^{5}= & \int_{\Omega=4 \pi W} \frac{\left[1-\exp \left(-\tau_{13}\right)\right]}{\tau_{13}} \exp \left[-\tau_{12}\left(R_{1}^{\prime \prime}\right)\right] \times \\
& \times \exp \left[-\tau_{12}\left(R_{2}^{\prime \prime}\right)\right] \exp \left[-\tau_{13}\left(R^{\prime \prime \prime}\right)\right] \mathbf{l} \frac{\mathrm{d} \omega}{4 \pi},
\end{aligned}
$$

the physical meaning of the different terms appearing in these integrals being the same as that mentioned in Section 3(в) for the escape probabilities $\beta_{12}^{5}$ and $\beta_{13}^{5}$.

The contributions $\mathbf{F}_{12}^{D}$ and $\mathbf{F}_{13}^{D}$, expressed in (63) and (65), correspond to the radiative forces due to the interactions between atoms at the fixed point $R$ and the photons emitted from the distant points $R_{1}^{\prime}, R_{2}^{\prime}, R^{\prime \prime \prime}$ and $R_{1}^{\prime \prime}, R_{2}^{\prime \prime}, R^{\prime \prime \prime}$. Because of the analogy between the expressions of the radiative forces $\mathbf{F}_{12}^{D}$ and $\mathbf{F}_{13}^{D}$ and those of the contributions $J_{12}^{2}$ and $J_{13}^{2}$ (see relations (35) and (38)), we also refer the reader to Section 3 (в) for the physical meaning of the different quantities appearing in expressions (63) and (65). 
We have chosen to define the following quantities (cf. Section 4(в))

$$
\begin{aligned}
F R E & =\log _{10}\left(\mathbf{F}^{R E} \cdot \mathbf{n} / K\right), \\
F C 12 & =\log _{10}\left(\mathbf{F}_{12}^{C} \cdot \mathbf{n} / K\right), \\
F D 12 & =\log _{10}\left(\left|\mathbf{F}_{12}^{D} \cdot \mathbf{n} / K\right|\right), \\
F C 13 & =\log _{10}\left(\mathbf{F}_{13}^{C} \cdot \mathbf{n} / K\right), \\
F D 13 & =\log _{10}\left(\left|\mathbf{F}_{13}^{D} \cdot \mathbf{n} / K\right|\right),
\end{aligned}
$$

in order to illustrate the behaviour of the radiative forces $\mathbf{F}^{R E}, \mathbf{F}_{12}^{C}, \mathbf{F}_{12}^{D}, \mathbf{F}_{13}^{C}$ and $\mathbf{F}_{13}^{D}$ across a D.E envelope.

\section{(C) Numerical applications}

We have illustrated in Figures 18-23 the behaviour of the different contributions $L G S_{12}, L G S_{12}^{5}, L G S_{12}^{2}, L G S_{13}, L G S_{13}^{5}$ and $L G S_{13}^{2}$ defined in (60), and in Figures 24-26 that of the contributions FRE, FC12, FD12, FC13 and FD13 defined in (67), as a function of the distance $L$ for a small sample of different physical and geometrical conditions prevailing in D.E envelopes.

While it would have been of great interest to present a larger number of numerical applications in order to discuss quantitatively the influences of the decelerating parameter $l$, the relative separation $\Delta X_{23}$ of the upper atomic levels, the distributions of the fictive opacities $\tau_{12}, \tau_{13}$, the maximal extension $L_{\max }$ of the medium, etc., on the behaviour of the source functions and radiative forces in D.E envelopes, we had to restrict ourselves because of the enormous amount of figures and space it would have required in the present paper. However, this unpublished work does exist (Surdej, 1979b - later referred to as Paper V) and could be obtained on simple request. Therefore, in the next section we shall only quote the most important results based on that general study with some references specific to Figures 18-26.

TABLE II

Caption for Figures 18-26

\begin{tabular}{llclc}
\hline Figure & Physical quantities & $l$ & $\Delta X_{23}$ & $\tau_{12}^{l}$ \\
\hline 18 & $L G S_{12}, L G S_{12}^{5}, L G S_{12}^{2}$ & 1 & 0.01 & 1 \\
19 & $L G S_{13}, L G S_{13}^{5}, L G S_{13}^{2}$ & 1 & 0.01 & 1 \\
20 & $L G S_{12}, L G S_{12}^{5}, L G S_{12}^{2}$ & 1 & 0.25 & 1 \\
21 & $L G S_{13}, L G S_{13}^{5}, L G S_{13}^{2}$ & 1 & 0.25 & 1 \\
22 & $L G S_{12}, L G S_{12}^{5}, L G S_{12}^{2}$ & 2 & 0.25 & 1 \\
23 & $L G S_{13}, L G S_{13}^{5}, L G S_{13}^{2}$ & 2 & 0.25 & 1 \\
24 & $F R E, F C 12, F D 12, F C 13, F D 13, F D$ & 1 & 0.01 & 1 \\
25 & $F R E, F C 12, F D 12, F C 13, F D 13, F D$ & 1 & 0.25 & 1 \\
26 & $F R E, F C 12, F D 12, F C 13, F D 13, F D$ & 2 & 0.25 & 1 \\
\hline
\end{tabular}



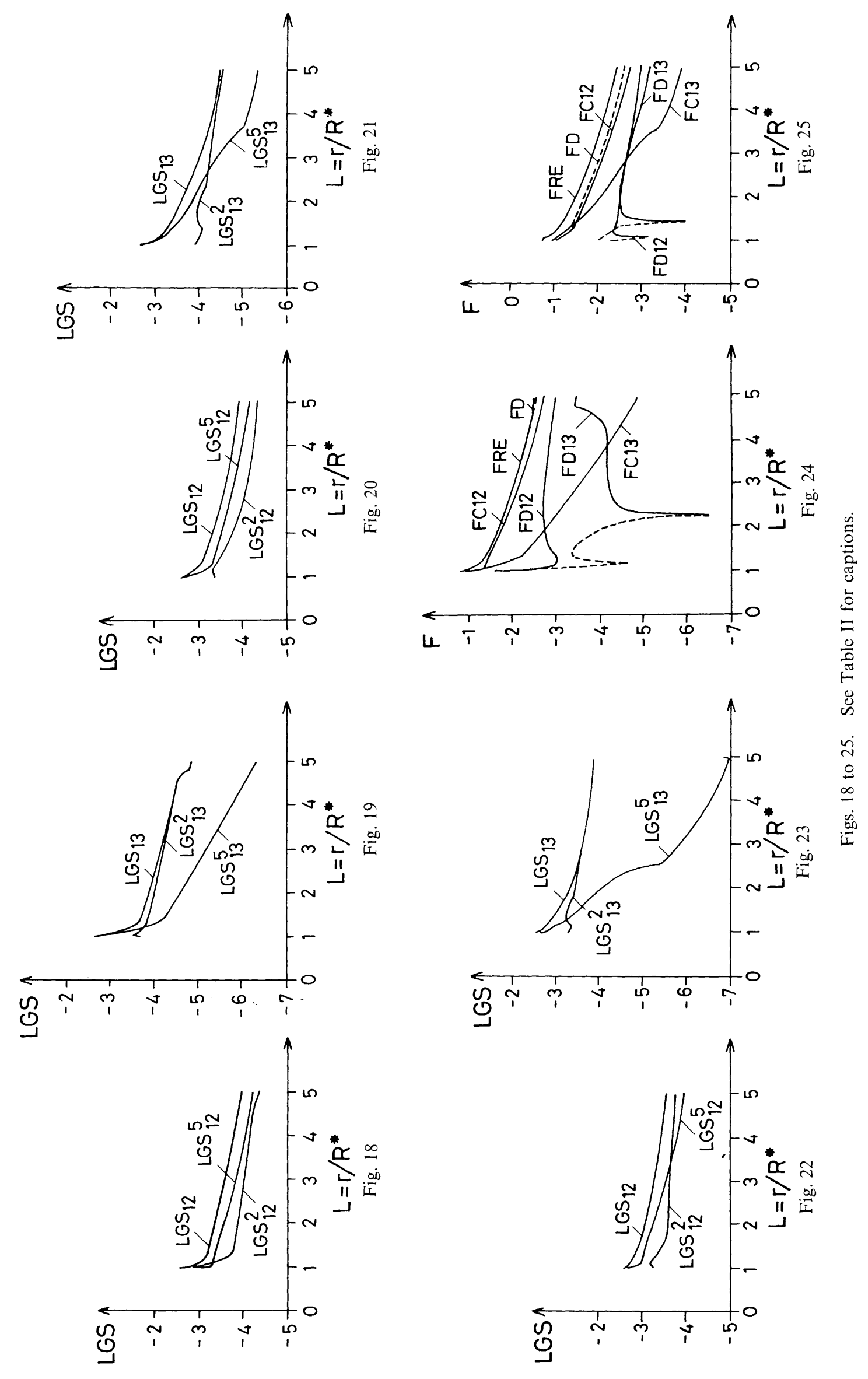


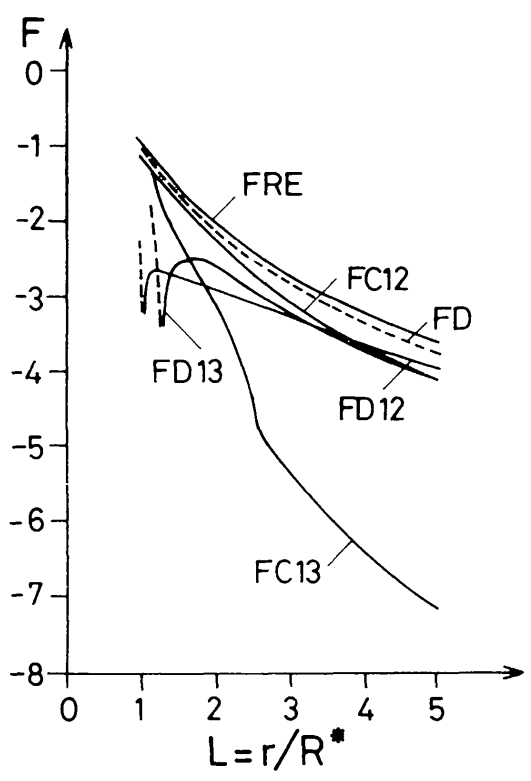

Fig. 26. See Table II for caption.

When the products $\mathbf{F}_{12}^{D} \cdot \mathbf{n}$ and $\mathbf{F}_{13}^{D} \cdot \mathbf{n}$ are negative - i.e., the radiative forces $\mathbf{F}_{12}^{D}$ and $\mathbf{F}_{13}^{D}$ are directed radially inward - the corresponding quantities $F D 12$ and FD13 are represented in Figures 24-26 with dashed lines.

Table II was constructed in an identical form to Table I, and constitutes the captions for Figures 18-26. Let us note, however, that for these numerical applications we fixed the maximal extension of the envelope to $L_{\max }=5$. We still chose the intensity $I_{c}$ such that $\lambda_{12} T=0.28979 \mathrm{~cm} \mathrm{deg}$ (Wien's law) with $\lambda_{12}=1.5 \times 10^{-5} \mathrm{~cm}$.

When calculating the values of the source functions $S_{12}$ and $S_{13}$ at a fixed point $R$ in a D.E envelope, we have seen that it was necessary to solve the set of Equations (59) in which each right-hand side depends simultaneously on the physical properties of the medium at $R$ and at distant points $R^{\prime \prime \prime}, R_{1}^{\prime}, R_{2}^{\prime}, R_{1}^{\prime \prime}$ and $R_{2}^{\prime \prime}$. As for the case of the two-level atom model treated in Paper II, we used an iterative numerical approach which, in the first approximation, neglects the distant radiative interactions and then replaces at the $n$th iteration the values of the source functions $S_{12}, S_{13}$ and of the fictive opacities $\tau_{12}, \tau_{13}$ in the terms of radiative coupling (see relations (33)(38)) by those calculated at the $(n-1)$ th iteration. This numerical approach converges rapidly and when computing all integrals $\left(\beta_{12}^{1}, \beta_{12}^{5}, J_{12}^{5}, J_{12}^{2}\right.$, etc.) with 200 steps at successive points separated by a distance $\Delta L=0.1$, eight iterations give a precision better than $0.1 \%$ for the values of the source functions $S_{12}, S_{13}$ across the medium. An approximate CPU time of seven minutes was necessary on a CDC 7600 computer for calculating the degree of excitation $\left(S_{12}, S_{13}\right)$, the radiative forces and one resonance doublet profile divided into 200 frequency intervals, in a D.E envelope with $L_{\max }=5$ and $\Delta L=0.1$. Let us point out that this numerical model can also take into account the effects of collisional excitation, limb-darkening and ionization whenever these are specified. For comparison, the calculations of the same 
quantities in the same conditions require about one minute of CPU time when using the two-level atom model.

In order to show the deviations between the resulting radiative force $\mathbf{F}^{R E}$ and the $\mathbf{F}_{12}^{D E}$ one (see relation (22) in Paper III) calculated for a two-level atom model characterized by an oscillator strength $f_{12}^{\prime}=f_{12}+f_{13}$, and thus $\tau_{12}^{l^{\prime}}=3 \tau_{12}^{l}$, and its statistical weights $g_{1}^{\prime}=2$ and $g_{2}^{\prime}=6$, we have also illustrated in Figures 24-26 (see the dashed lines) the corresponding quantity $F D$ defined by

$$
F D=\log _{10}\left(\mathbf{F}_{12}^{D E} \cdot \mathbf{n} / K\right)
$$

(D) Discussion of THE Results

As expected, the behaviour of the source functions $S_{12}$ and $S_{13}$ characterizing the degree of excitation of a three-level atom (resonance doublet) critically depends on the complex radiative coupling between distant atoms in a D.E envelope. Indeed, we notice immediately from Figures 18-23 that the contributions $L G S_{12}^{2}$ and $L G S_{13}^{2}$ due to the distant radiative interactions are very competitive, even in some cases dominant, when compared to the contributions $L G S_{12}^{5}$ and $L G S_{13}^{5}$ associated with the source of stellar radiation.

In order to better understand the role of the additional radiative interactions between distant atoms situated at points $R, R^{\prime}$ and $R, R^{\prime \prime}$, we compared the results of all our numerical applications (see Paper V), including the results presented in Figures $18-23$, to those directly obtained for the two-level atom model $\left(f_{12}^{\prime}=3 f_{12}\right.$, $g_{1}^{\prime}=2, g_{2}^{\prime}=6$ ). In the following, we shall call $L G S 2$ the contribution to the source function $L G S$ of the two-level atom model due to the distant interactions between atoms at points $R, R^{\prime \prime \prime}$ and $L G S 5$ the one associated with the source of stellar radiation. Let us point out immediately that in both cases of optically thick $\left(\tau_{12}>1, \tau_{13}>1, \tau_{12}^{\prime}>1\right)$ and optically thin $\left(\tau_{12}<1, \tau_{13}<1, \tau_{12}^{\prime}<1\right)$ approximations, the quantities $L G S_{12}, L G S_{12}^{2}, L G S_{12}^{5}$ and $L G S_{13}, L G S_{13}^{2}, L G S_{13}^{5}$ which would have been calculated by neglecting the additional radiative interactions between distant atoms at points $R, R^{\prime}$ and $R, R^{\prime \prime}$, would then be perfectly represented by the respective quantities $L G S, L G S 2, L G S 5$.

After comparing the results of all our numerical applications (cf. Paper V) we found the following general relations

$$
L G S_{12}>L G S
$$

because

$$
L G S_{12}^{5} \simeq L G S 5
$$

and

and that

$$
L G S_{12}^{2}>L G S 2
$$

$$
L G S 13<L G S
$$

because

$$
L G S_{13}^{5} \ll L G S 5
$$


while

$$
L G S_{13}^{2}>L G S 2 \text {. }
$$

From (69) and (72) we conclude that the additional radiative coupling between atoms situated at a fixed point $R$ in the envelope and the photons emitted with a local frequency $v_{L}^{\prime} \simeq v_{13}$ (resp. $\left.v_{L}^{\prime \prime} \simeq v_{12}\right)$ from the geometrical locus $\left(R, R^{\prime}\right)$ (resp. $\left(R, R^{\prime \prime}\right)$ ) has the resulting effect to increase (resp. decrease) the population of the upper atomic level 2 (resp. 3).

As indicated before, we shall not give here a quantitative interpretation of these results. However, we shall discuss briefly their physical meaning.

The inequalities (71) and (74) are mainly interpreted in that the fraction of space $\left[\Omega\left(R, R^{\prime \prime \prime}\right) \cup \Omega\left(R, R^{\prime}\right)\right] / 4 \pi$ (resp. $\left.\left[\Omega\left(R, R^{\prime \prime \prime}\right) \cup \Omega\left(R, R^{\prime \prime}\right)\right] / 4 \pi\right)$ intercepting the geometrical loci $\left(R, R^{\prime \prime \prime}\right)$ and $\left(R, R^{\prime}\right)$ (resp. $\left.\left(R, R^{\prime \prime}\right)\right)$, as seen by a fixed observer at $R$, is greater than the single fraction of space $\Omega\left(R, R^{\prime \prime \prime}\right) / 4 \pi$.

We have seen in Paper II that the occultation of the stellar disk by the geometrical locus $\left(R, R^{\prime \prime \prime}\right)$, as seen by a fixed observer at $R$, was negligible unless $R$ lies very close to the star. A fortiori, this is also true for the occultation of the stellar disk by the geometrical locus $\left(R, R^{\prime}\right)$ which is located outside the geometrical locus $\left(R, R^{\prime \prime \prime}\right)$ in the regions III and IV of the envelope (see Figure 6). Therefore, the contributions $L G S_{12}^{5}$ and $L G S 5$ associated with the source of excitation due to the stellar radiation are comparable in most parts of the envelope (see inequality (70)). Let us remark, however, that below the distance $L^{*}$, at which the solid angle intercepting the geometrical locus $\left(R, R^{\prime \prime \prime}\right)$ is equal to the solid angle $4 \pi W$ subtending the stellar disk - i.e.,

$$
L^{*}=\sqrt{\frac{l+1}{l}},
$$

we evidently have $L G S_{12}^{5} \lesssim L G S 5$, unless the relative separation $\Delta X_{23}$ of the upper levels is appreciable and in which case we have $L G S_{12}^{5} \gtrsim L G S 5$ (see $L G S_{12}^{5}$ in Figures 18 and 20).

Because the occultation of the stellar disk by the geometrical locus $\left(R, R^{\prime \prime}\right)$ (see Figures 6-9) is much more important than that due to the geometrical locus $\left(R, R^{\prime \prime \prime}\right)$, the inequality (73) is simply interpreted. Setting $L^{\prime \prime}=1, \theta^{\prime \prime}=\pi$ and $\theta=\pi$ in relation (29), we easily find that the distance $L_{0}$ at which the occultation of the stellar disk by the geometrical locus $\left(R, R^{\prime \prime}\right)$ starts is given by

$$
L_{0} \simeq\left(1-\Delta X_{23}\right)^{-1 / l}
$$

and that consequently the contribution $L G S_{13}^{5}$ will be affected by that occultation closer to the star as $\Delta X_{23}$ is smaller or $l$ greater (see Figures 19, 21 and 23). Furthermore, because the cross-section of the geometrical locus $\left(R, R^{\prime \prime}\right)$ projected onto the stellar disk appears more important as $\Delta X_{23}$ decreases and/or $l$ increases and because the absorption of the stellar continuum then takes place at points $R^{\prime \prime}$ situated closer 
to $R$, the different behaviours observed for the contribution $L G S_{13}^{5}$ in Figures 19 , 21 and 23 are easily understood.

Finally, from the large set of numerical applications we performed (cf. Paper V), we derived the additional expected conclusions:

(i) When the relative separation $\Delta X_{23}$ of the upper atomic levels becomes very small (for instance, $\Delta X_{23}=0.001$ ) the source functions $S_{12}, S_{13}$ and $S_{12}^{\prime}$, respectively, calculated by means of the three-level and two-level atom models are such that relation (52) is fulfilled.

(ii) When $\Delta X_{23} \rightarrow 1+X_{c}$, where $X_{c}$ is the 'total occultation' frequency defined below in (101), the source functions $S_{12}$ and $S_{13}$ calculated for the three-level atom model become identical to those directly calculated by means of the twolevel atom model for the respective values $f_{12}^{\prime}=f_{12}, g_{1}^{\prime}=2, g_{2}^{\prime}=2$ and $f_{12}^{\prime}=$ $f_{13}, g_{1}^{\prime}=2, g_{2}^{\prime}=4$. This result can be easily understood because when $\Delta X_{23}>$ $1+X_{c}$ the radiative interactions between distant atoms situated at points $R, R^{\prime}$ and $R, R^{\prime \prime}$ completely vanish.

(iii) When comparing the behaviour of the quantities $L G S_{12}^{2}$ and $L G S_{13}^{2}$ for a similar value of $\Delta X_{23}$ as a function of the decelerating parameter $l$ (or $G$ ) (see, for instance, Figures 21 and 23), we conclude that the radiative interactions between distant atoms are more efficient as the decelerating parameter of the envelope is greater. Recalling (see Paper II) that the fraction of space $\Omega\left(R, R^{\prime \prime \prime}\right) / 4 \pi$ intercepting the geometrical locus $\left(R, R^{\prime \prime \prime}\right)$ increases with the value of the decelerating parameter $l$ (or $G$ ) and noting (see Figures 6-9) that this is also the case for the fraction of space $\Omega\left(R, R^{\prime}\right) / 4 \pi$ (resp. $\left.\Omega\left(R, R^{\prime \prime}\right) / 4 \pi\right)$ intercepting the geometrical locus $\left(R, R^{\prime}\right)$ (resp. $\left(R, R^{\prime \prime}\right)$ ), these results are interpreted here in a simple way.

(iv) In the optically thin approximation $\left(\tau_{12}<1, \tau_{13}<1\right)$ it appears that the contributions $L G S_{12}^{2}$ and $L G S_{13}^{2}$ to the source functions $S_{12}$ and $S_{13}$ are negligible when compared to $L G S_{12}^{5}$ and $L G S_{13}^{5}$, and we reach the same conclusion as in Paper II for a single line transition; namely, that the radiative interactions between distant atoms situated at points $R, R_{1}^{\prime}, R_{2}^{\prime}, R_{1}^{\prime \prime}, R_{2}^{\prime \prime}$ and $\mathrm{R}^{\prime \prime \prime}$ become important only when the medium is optically thick to the spectral line radiation.

In the remainder of this section we shall briefly discuss the behaviour of the radiative forces $\mathbf{F}^{R E}, \mathbf{F}_{12}^{C}, \mathbf{F}_{12}^{D}, \mathbf{F}_{13}^{C}, \mathbf{F}_{13}^{D}$ and $\mathbf{F}_{12}^{D E}$, represented, respectively, by the quantities $F R E, F C 12, F D 12, F C 13, F D 13$ and $F D$ (see relations (67) and (68)) in Figures 24-26, on the basis of the large number of numerical applications we performed (cf. Paper V).

As previously, we compared the results of all these numerical applications, including the results in Figures 24-26, to those directly obtained by means of the two-level atom model $\left(f_{12}^{\prime}=3 f_{12}, g_{1}^{\prime}=2, g_{2}^{\prime}=6\right)$. In the following, we shall call $F C D$ and $F D D$ (cf. relations (26) and (27) in Paper III) those contributions to the radiative 
force $F D$ (see relation (68)) due to the source of stellar photons and to the radiative interactions between distant atoms situated at points $R, R^{\prime \prime \prime}$.

From a comparison of these results, we then find that the following relations apply in most parts of the envelope (i.e., for $L>\max \left(L_{0}, L^{*}\right)$, see relations (75) and (76))

$$
\begin{aligned}
& F C 12 \simeq F C D, \\
& F D 12>F D D, \\
& F C 13 \ll F C D, \\
& F D 13>F D D .
\end{aligned}
$$

It is not surprising to notice the analogy existing between these inequalities and those in (70), (71), (73) and (74) because the expressions of the radiative forces $\mathbf{F}_{12}^{C}, \mathbf{F}_{12}^{D}$, $\mathbf{F}_{13}^{C}, \mathbf{F}_{13}^{D}$, projected along the radial direction $\mathbf{n}$, only differ from the quantities $J_{12}^{5}$, $J_{12}^{2}, J_{13}^{5}, J_{13}^{2}$ (see relations (33)-(38)) by the presence of the factor $(4 \pi / c) k_{12}$ or $(4 \pi / c) k_{13}$ and the product $\mathbf{I} \cdot \mathbf{n}$ included under the integral sign of the expressions (62)-(66). Consequently, a reasoning analogous to the one followed in interpreting physically the inequalities (69)-(74) enables us to understand easily the inequalities (77)-(80).

As for the case of a single line transition (see Paper III), we notice in Figures 24-26 that the radiative forces $\mathbf{F}_{12}^{D}$ and $\mathbf{F}_{13}^{D}$ resulting from the distant interactions are directed radially inward in the close neighbourhood of the star, but remain in amplitude much smaller than the contributions $\mathbf{F}_{12}^{C}$ and $\mathbf{F}_{13}^{C}$ due to the stellar continuum. Because near the stellar core $\left(L<L_{0}\right)$, the resulting impulsion carried by the photons emitted with a local frequency $v_{L}^{\prime \prime} \simeq v_{12}$ from the geometrical locus $\left(R, R^{\prime \prime}\right)$ (see the region I in Figure 6) is totally directed radially inward, the radiative force $\mathbf{F}_{13}^{D}$ drops to zero further than does the contribution $\mathbf{F}_{12}^{D}$ (see Figures 24-26).

From analogy with the results derived in Section 4(D) (cf. results (53), (54)), we can easily show that if the medium is optically thick to the spectral line radiation $\left(\tau_{12}>1\right)$

$$
\begin{aligned}
& F C 12 \simeq F C D, \\
& F C 13 \simeq F C D, \quad \text { for } L \in\left[1, L_{0}\right] .
\end{aligned}
$$

We immediately conclude that in an optically thick D.E envelope, the radiative force $\mathbf{F}^{R E}$ acting on an atom, via the resonance transitions $1 \rightleftharpoons 2$ and $1 \rightleftharpoons 3$, is at least two times higher in the spatial interval $L \in\left[1, L_{0}\right]$, and higher in the spatial interval $L \in\left[L_{0}, L_{\max }\right]$, than the radiative force $\mathbf{F}_{12}^{D E}$ calculated by supposing that the components of a resonance doublet form a single line transition (see Figures 25 and 26).

As expected, when the relative separation $\Delta X_{23}$ of the upper atomic levels becomes very small (cf. $\Delta X_{23}=0.01$ in Figure 24 ), the resulting radiative force $\mathbf{F}^{R E}$ is well represented by the force $\mathbf{F}_{12}^{D E}$ directly calculated by means of the two-level atom model.

If the medium is optically thin to the spectral line radiation $\left(\tau_{12}<1, \tau_{13}<1\right.$, 
$\tau_{12}^{\prime}<1$ ), we have shown before that the distant radiative interactions vanish and so do the contributions $\mathbf{F}_{12}^{D}$ and $\mathbf{F}_{13}^{D}$. As in the case of an A.E envelope, it is easy to state that the resulting radiative forces $\mathbf{F}^{R E}$ and $\mathbf{F}_{12}^{D E}$, calculated respectively by means of the three-level and two-level atom models, do present the same behaviour (cf. relations (56) and (57)).

We can summarize all the preceding conclusions by saying that in a D.E envelope, besides the extreme cases $\Delta X_{23} \ll 1$ or $\tau_{12}<1, \tau_{13}<1$ for which $\mathbf{F}^{R E} \simeq \mathbf{F}_{12}^{D E}$, the resulting radiative force $\mathbf{F}^{R E}$ acting on an atom, via the resonance transitions $1 \rightleftharpoons 2$ and $1 \rightleftharpoons 3$, is generally about twice as high as the force $\mathbf{F}_{12}^{D E}$ calculated by supposing that the resonance doublet forms a single line transition.

Bertout (1977), and Wolf et al. (1977) have attempted to interpret quantitatively the formation of inverse $\mathbf{P}$ Cygni profiles in inward-accelerating envelopes (later referred to as I.A envelopes) around YY Orionis stars. The velocity distributions $v(r)$ characterizing these free-infalling media being mathematically identical to those for D.E envelopes, with the only difference that the velocity vector $\mathbf{v}(r)$ is directed radially inward, it follows that the expressions of the radiative force $\mathbf{F}^{R E}$, derived here for a resonance doublet, and $\mathbf{F}_{i j}^{D E}$, derived in Paper III for a single line transition $i \rightleftharpoons j$, do apply integrally to the case of I.A envelopes. Therefore, it is likely that the great variability of some YY Orionis profiles reported by Mundt and Wolf (1977), Walker (1977) and Appenzeller (1979) is partly due to dynamical instabilities developed by the radiative forces $\mathbf{F}^{R E}, \mathbf{F}_{i j}^{D E}$ which act against the free-infall of the surrounding medium.

\section{Formation of Resonance Doublet Profiles in A.E Envelopes}

\section{(a) General equations}

From the large Doppler shifts of the absorbing and emitting atoms across an expanding medium, there results a new energy distribution in frequency of the stellar continuum in the form of a 'resonance doublet profile'. This profile lies within the frequency interval $v \in\left[v_{f}, v_{i}\right]$, where $v_{f}$ (resp. $v_{i}$ ) corresponds to the extreme frequency, as seen by a fixed observer, of the photons emitted with a local frequency $v_{L} \simeq v_{12}$ (resp. $v_{L} \simeq v_{13}$ ) by the atoms receding from (resp. approaching) the observer with the maximal expansion velocity $-v_{\max }$ (resp. $v_{\max }$ ).

Let $X$ be the dimensionless frequency defined by

$$
X=\frac{\left(v-v_{12}\right)}{\left(v_{f}-v_{12}\right)},
$$

such that the central frequency $X=0$ corresponds to the unshifted position of the red component $(1 \rightleftharpoons 2)$ of the resonance doublet. With the use of relations $(9),(15)$, (17) and (82) it is then easy to show that the frequencies $X_{i}, X_{f}$, associated to $v_{i}$ and $v_{f}$, are expressed by

$$
X_{i}=-\left(\frac{v_{13}}{v_{12}}+\Delta X_{23}\right)
$$


and $X_{f}=1$, and since $v_{13} / v_{12} \simeq 1$, we find with a good approximation that the frequency interval in which a resonance doublet profile is formed is given by

$$
X \in\left[-1-\Delta X_{23}, 1\right] .
$$

Similarly, if we had chosen to define the frequency interval $Y \in\left[Y_{i}, Y_{f}\right]$ in which is formed a resonance doublet profile such that the central frequency $Y=0$ corresponds to the unshifted position of the blue component $(1 \rightleftharpoons 3)$ of the resonance doublet, we would have found that

$$
Y \in\left[-1,1+\Delta X_{23}\right],
$$

and that the relation existing between the frequencies $X$ and $Y$ is, of course, given by

$$
Y=X+\Delta X_{23} .
$$

In the following, we establish the general expression $E(X) / E_{c}$ of a resonance doublet profile formed in an A.E envelope. In the frequency interval $X \in\left[-1-\Delta X_{23}, 1\right]$, the expression $E(X) / E_{c}$ represents merely the total amount of spectral energy $E(X)$, defined per frequency and solid angle units, radiated by the medium towards a fixed observer, and which has been normalized to the flux $E_{c}$ of the stellar continuum $I_{c}$ integrated over the surface of the central core of the star; i.e.,

$$
E_{c}=\pi R^{* 2} I_{c} .
$$

The quantity $E(X)$ is itself given by the integration of the sum of the monochromatic intensity functions $I_{12}(X)$ and $I_{13}\left(Y=X+\Delta X_{23}\right)$, implicitly space-defined, over a plane perpendicular to the line of sight

$$
E(X)=\iint_{\Sigma}\left[I_{12}(X)+I_{13}\left(Y=X+\Delta X_{23}\right)\right] \mathrm{d} \sigma .
$$

In the frame of a fixed observer, $I_{12}(X)$ and $I_{13}(Y)$ account, respectively, for the specific intensities of line radiation emitted, with a local frequency $v_{L} \simeq v_{12}$ and $v_{L}^{\prime} \simeq v_{13}$ along a direction parallel to the line of sight, by those parts of the envelope presenting a constant Doppler shift equal to

and

$$
X=-v_{s} / v_{\max }
$$

$$
Y=-v_{s}^{\prime} / v_{\max },
$$

where $v_{s}$ and $v_{s}^{\prime}$ denote the relative velocity of the envelope at the corresponding points with respect to the observer. We solved this set of equations for different values of the frequency $X \in\left[-1-\Delta X_{23}, 1\right]$ and the resulting 'surfaces of equal frequencies $X$ and $Y=X+\Delta X_{23}$, as seen by a fixed observer are, respectively, illustrated with dashed and full lines in Figure $27(l=-0.5)$ and Figure $28(l=-2)$ for the values of the parameters $\Delta X_{23}=0.25$ and $L_{\max }=20$. 


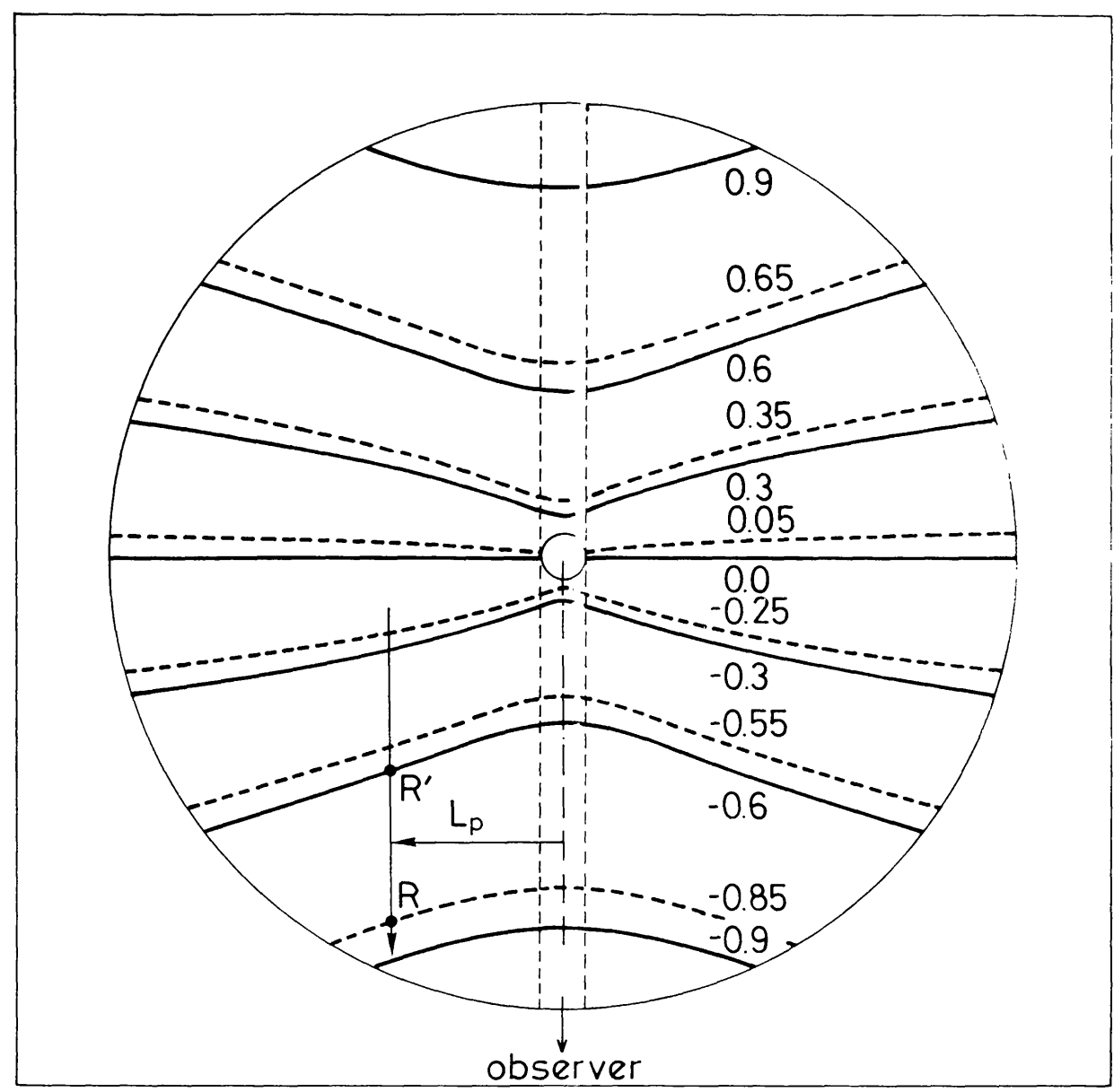

Fig. 27. Surfaces of equal frequencies $X=-0.85,-0.55,-0.25,0.05,0.35,0.65$ and $Y=X+\Delta X_{23}$ in an A.E envelope for the values of the parameters $l=-0.5, \Delta X_{23}=0.25$ and $L_{\max }=20$.

Following a similar reasoning to the one adopted in Paper IV when deriving the monochromatic intensity function $I(X)$ for the case of a single line transition $i \rightleftharpoons j$, we easily deduce the expressions for the intensities $I_{12}(X)$ and $I_{13}(Y)$ appearing in relation (87). We obtain

and

$$
I_{12}(X)=S_{12}\left[1-\exp \left(-\tau_{12}\right)\right]
$$

$$
I_{13}(Y)=S_{13}\left[1-\exp \left(-\tau_{13}\right)\right] \exp \left(-\tau_{12}\right) .
$$

In that relation, the quantities $S_{12}, \tau_{12}$ and $S_{13}, \tau_{13}$ are, respectively, evaluated at the points $R$ and $R^{\prime}$ (see Figure 27) defined by the intersections of the 'surfaces of equal frequencies $X$ and $Y$ ' and the axis parallel to the line of sight for a given value of the impact parameter $L p$. Let us note that in the last relation we have explicitly taken into account the fact that the spectral line radiation, emitted with a local frequency $v_{L}^{\prime} \simeq v_{13}$ at a point $R^{\prime}$ towards the observer, can be absorbed with a probability $\left[1-\exp \left(-\tau_{12}\right)\right]$ by the atoms situated in the neighbourhood of the distant point $R$ (see Figure 27). Furthermore, we adopt the convention that if for some values of the frequency $X \in\left[-1-\Delta X_{23}, 1\right]$ and of the impact parameter 


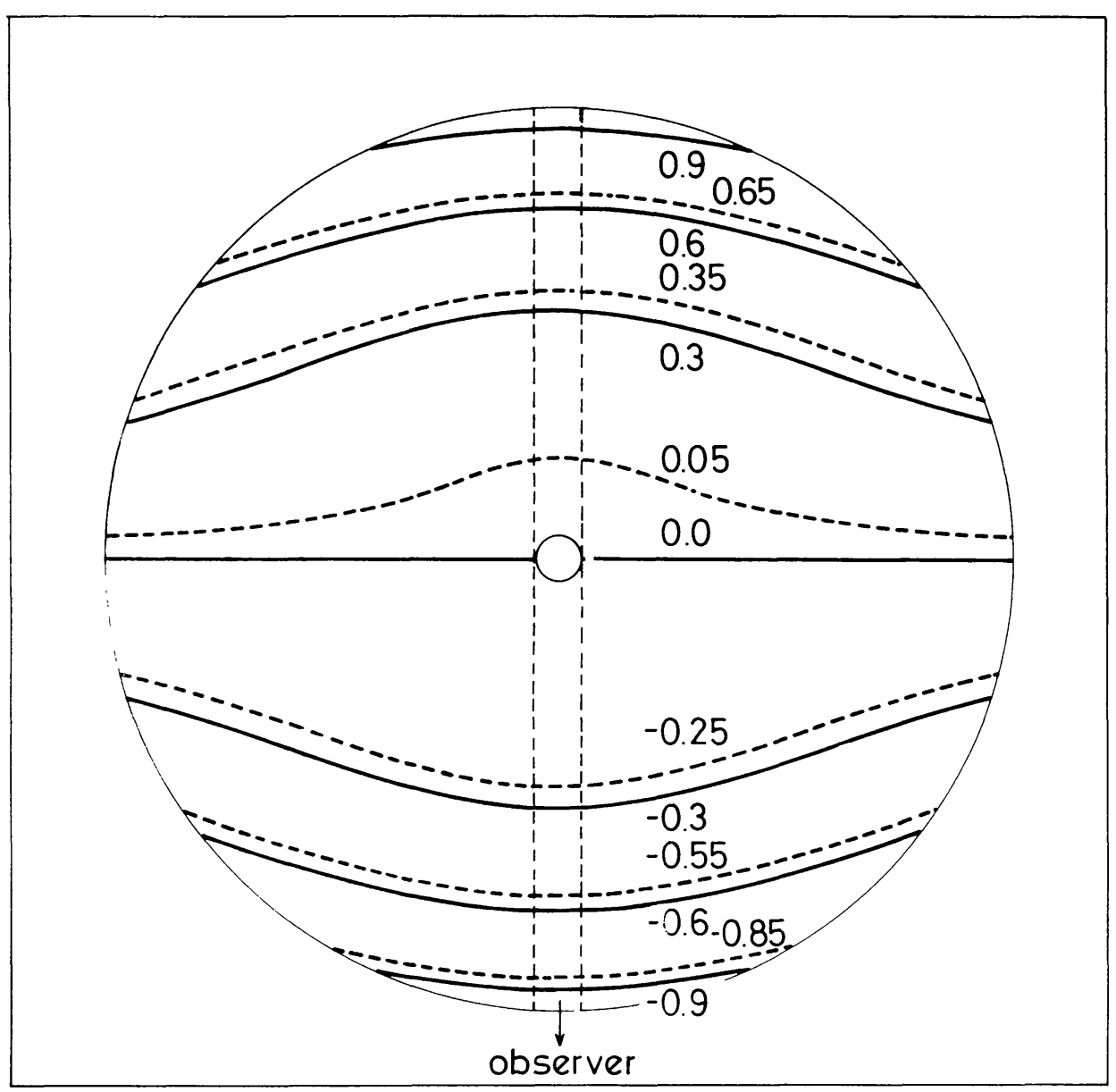

Fig. 28. Surfaces of equal frequencies $X=-0.85,-0.55,-0.25,0.05,0.35,0.65$ and $Y=X+\Delta X_{23}$ in an A.E envelope for the values of the parameters $l=-2, \Delta X_{23}=0.25$ and $L_{\max }=20$.

$L p \in\left[0, L_{\max }\right]$, Equations (88) and/or (89) have no solutions, the fictive opacities $\tau_{12}$ and/or $\tau_{13}$ are set equal to zero in relation (90).

Concerning the contribution to the resonance doublet profile $E(X) / E_{c}$ due to the radiation emitted by the stellar core, we similarly find that

$$
I_{12}(X)+I_{13}(Y)=I_{c} \exp \left(-\tau_{12}\right) \exp \left(-\tau_{13}\right) .
$$

Finally, because of the symmetry of the envelope around the line of sight and with the help of relations (86), (87), (90) and (91), the expression of the resonance doublet profile formed in an A.E envelope takes the form

$$
\begin{aligned}
& \frac{E(X)}{E_{c}}=2\left[\int _ { 0 } ^ { L _ { \operatorname { m a x } } } \left(\frac{S_{12}}{I_{c}}\left[1-\exp \left(-\tau_{12}\right)\right]+\right.\right. \\
& \left.\quad+\frac{S_{13}}{I_{c}}\left[1-\exp \left(-\tau_{13}\right)\right] \exp \left(-\tau_{12}\right)\right) L p \mathrm{dLp}+ \\
& \left.\quad+\int_{0}^{1} \exp \left(-\tau_{12}\right) \exp \left(-\tau_{13}\right) L p \mathrm{~d} L p\right], \\
& \text { for } X \in\left[-1-\Delta X_{23}, 1\right] .
\end{aligned}
$$


(B) Numerical applications

In order to set forth clearly the dependence of resonance doublet profiles formed in A.E envelopes on the accelerating parameter $l$, on the relative separation $\Delta X_{23}$ of the upper atomic levels and on the distribution of the fictive opacities $\tau_{12}$ and $\tau_{13}$, we have solved numerically Equation (92) under different physical and geometrical conditions prevailing in those expanding media. The results of these calculations are illustrated in Figures 29-32. Each of these figures contains four graphs. The first displays the resulting profile function $E(X) / E_{c}$ against the frequency $X \in\left[-1-\Delta X_{23}, 1\right]$, while the other three inform about its individual contributions, respectively, $E_{1}(X) / E_{c}$, $E_{2}(X) / E_{c}$ and $E_{3}(X) / E_{c}$ which are formed in distinct parts of the medium as follows: the profiles $E_{1}(X) / E_{c}$ and $E_{2}(X) / E_{c}$ account for the emission of the spectral line radiation, in both resonance transitions $1 \rightleftharpoons 2$ and $1 \rightleftharpoons 3$, by atoms situated in the regions of the envelope located respectively inside and outside the axes parallel to the line of sight for the value of the impact parameter $L p=1$ (see the dashed lines in Figures 27 and 28). The profile $E_{3}(X) / E_{c}$ represents the contribution due to the radiation emitted by the stellar core which has been attenuated by the atoms situated between the star and the observer through radiative absorptions in the line transitions $1 \rightarrow 2$ and $1 \rightarrow 3$.

Table III summarizes the sets of parameters used for the calculations of each profile. In that table the different columns give, respectively, the number of the figure and the relevant values of the parameters $l, \Delta X_{23}$ and $\tau_{12}^{l}$.

For all numerical applications, we fixed the maximal extension of the envelope to $L_{\max }=20$. As previously, we have chosen the oscillator strengths such that $f_{13}=2 f_{12}$, and thus $\tau_{13}^{l}=2 \tau_{12}^{l}$, and the statistical weights of the atomic levels $g_{1}=2, g_{2}=2$ and $g_{3}=4$ in agreement with the atomic parameters of a typical resonance doublet $\left({ }^{2} S_{1 / 2}-{ }^{2} P_{1 / 2.3 / 2}^{0}\right)$.

In the first graph of Figures 30 and 31, we have also illustrated with dashed lines the resulting profile $E(X) / E_{c}$ obtained by neglecting the distant radiative interactions when evaluating the degree of excitation of the atoms across the medium. In other words, we assumed for the particular case $l=-1$ that the distributions of the source functions $S_{12}$ and $S_{13}$ are such that $S_{12}=S_{13}=I_{c} W$ (cf. Section 4(D)).

TABLE III

Caption for Figures 29-32

\begin{tabular}{llcc}
\hline Figure & \multicolumn{1}{c}{$l$} & $\Delta X_{23}$ & $\tau_{12}^{l}$ \\
\hline 29 & -0.5 & 0.25 & 10 \\
30 & -1 & 0.25 & 100 \\
31 & -1 & 0.25 & $10^{3}$ \\
32 & -2 & 0.50 & $10^{5}$ \\
\hline
\end{tabular}



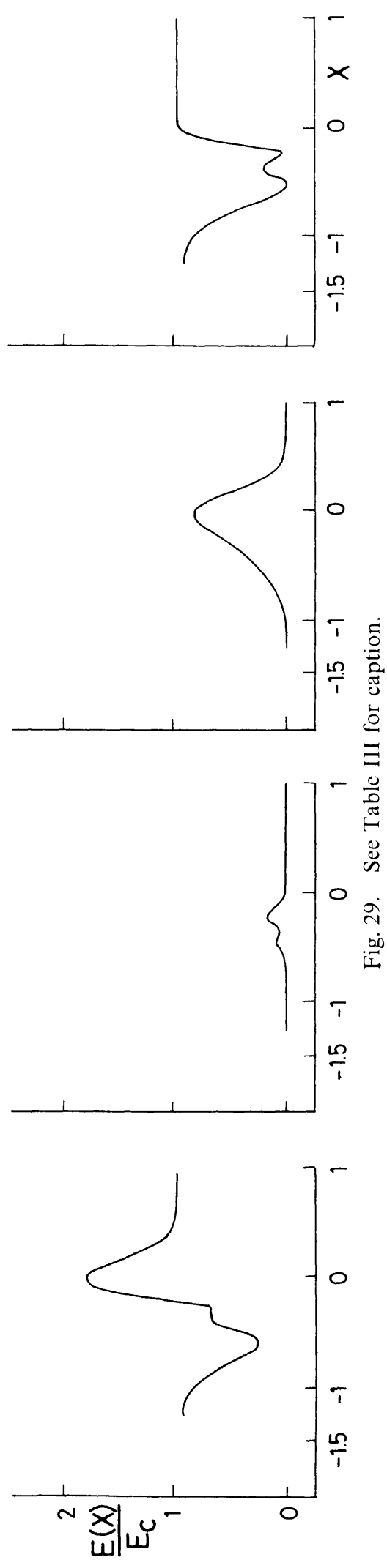
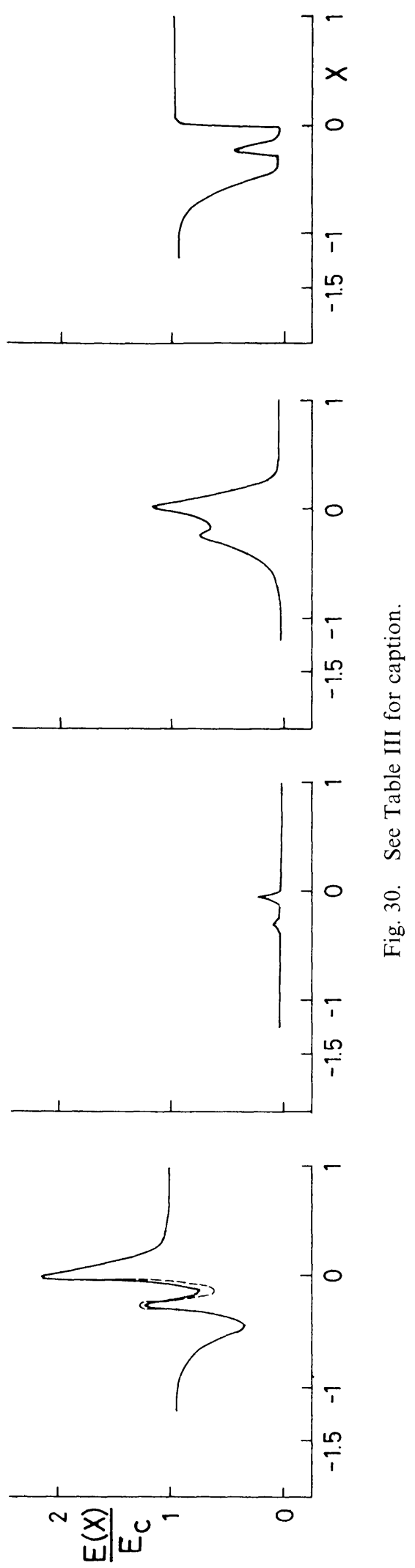

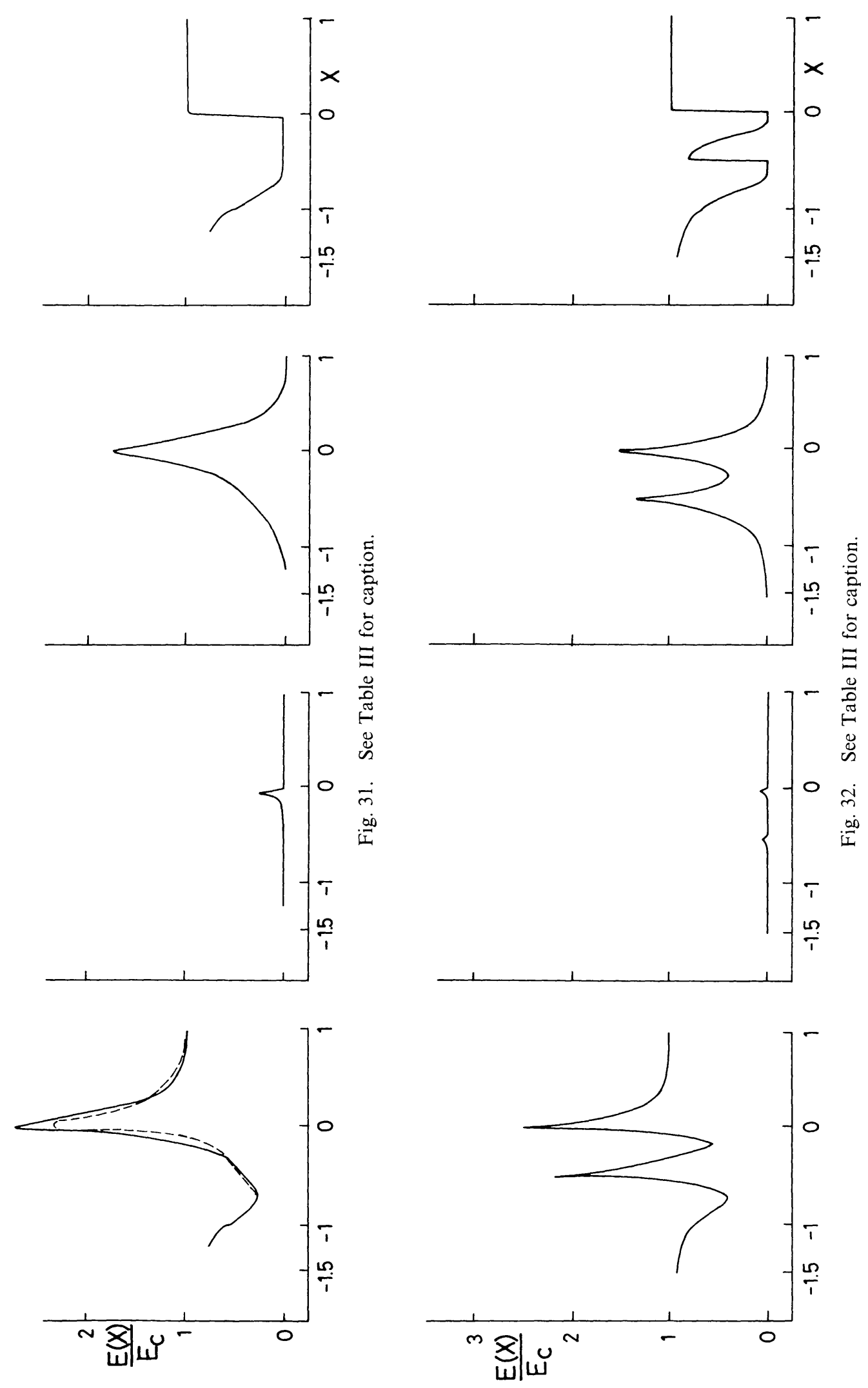


\section{(c) Discussion of the Results}

Inspection of Figures 29-32 shows that resonance doublet profiles formed in A.E envelopes range between single and double P Cygni profiles.

First, the importance of taking into account the radiative interactions between distant atoms when calculating resonance doublet profiles in an A.E envelope can be directly seen by comparing the full and dashed lines in the first graph of Figures 30 and 31 . From these and additional results, we do systematically observe that the profiles calculated rigorously present, around the central frequency $X=0$, intensities higher than those obtained by neglecting the role of the distant interactions between the atoms situated at a fixed point $R$ and the photons emitted with a local frequency $v_{L}^{\prime} \simeq v_{13}$ from the geometrical locus $\left(R, R^{\prime}\right)$, whereas in the rest of the spectral interval $X \in[-1,1]$ it is the reverse. These results can be easily understood if we remember (see Section 4(D)) that in an A.E envelope, the distant radiative interactions have the effect of increasing the population of the upper atomic level 2 in the approximate spatial interval $L \in\left[1, L_{1}\right]$ (see relation (50)), whereas for $L \in\left[L_{1}, L_{2}\right]$ (see relation (51)), these interactions being less efficient, the population of that same level appears slightly decreased. Because for $|X|>\left|X\left(L_{1}\right)\right|$ the surfaces of equal frequency $X$ are entirely confined to the spatial interval $L \in\left[L_{1}, L_{\mathrm{max}}\right]$ (see Figures 27 and 28), we can understand that in the frequency interval $\left|X\left(L_{2}\right)\right|>X>\left|X\left(L_{1}\right)\right|$ the profiles calculated rigorously by means of Equation (92) present intensities slightly smaller than those associated with the profiles obtained by neglecting the distant interactions. Similarly for $|X|<\left|X\left(L_{1}\right)\right|$, most of the contribution to the emission profile $E(X) / E_{c}$ is due to the spectral radiation emitted with a local frequency $v_{L} \simeq v_{12}$ from the regions of the surfaces of equal frequency $X$ located the closest to the stellar core, and the results illustrated in Figures 30 and 31 are easily interpreted. Combining relations (16), (50), (51) and (88), we find that the values of the frequencies $\left|X\left(L_{1}\right)\right|$ and $\left|X\left(L_{2}\right)\right|$ defined above are given by

$$
\left|X\left(L_{1}\right)\right|=L_{\max }^{l}+\Delta X_{23}
$$

and

$$
\left|X\left(L_{2}\right)\right|=\left(\tau_{13}^{l} L_{\max }^{2 l-1}\right)^{l /(2 l-1)}+\Delta X_{23} .
$$

For the values of the parameters $l, L_{\max }, \Delta X_{23}$ and $\tau_{13}^{l}$ corresponding to Figures 30 and 31 , we can verify that these last relations define correctly the spectral intervals in which deviations are observed between the profiles represented by full and dashed lines.

From Figures 29-32 it is interesting to note that for some values of the parameters $l, \Delta X_{23}$ and $\tau_{12}^{l}$, the absorption profile $E_{3}(X) / E_{c}$ is formed by two distinct absorption components, while for other values it is not. Defining by

$$
X_{\min }=-L_{\max }^{l}
$$

the frequency of the surface of equal frequency $X$ for which the occultation of the stellar core becomes total, we easily establish that the absorption profile $E_{3}(X) / E_{c}$ 
will be resolved into two components if, and only if, the conditions

$$
\tau_{12}\left(X=-\Delta X_{23}, \theta=0\right) \lesssim 1
$$

and

$$
\Delta X_{23}>-X_{\min }
$$

are fulfilled. The first of these conditions means that the medium must be optically thin to the spectral radiation in the resonance transition $1 \rightleftharpoons 2$ at a distance $L \geqslant$ $L\left(X=-\Delta X_{23}\right)$, where $L\left(X=-\Delta X_{23}\right)$ is defined by the intersection of the surface of equal frequency $X=-\Delta X_{23}$ and the radial direction. Indeed, if this were not the case (cf. Figure 31, $\tau_{12}(X=-0.25, \theta=0)=8$ ), the absorption profile would remain saturated at the value $\exp \left[-\tau_{12}\left(X=-\Delta X_{23}, \theta=0\right)\right] \ll 1$ in the frequency interval $X \in\left[-\Delta X_{23}, X_{\min }\right]$ and, consequently, it would appear as formed by a unique absorption component. The second condition implies that the occultation of the stellar core by the surfaces of equal frequency $Y=X+\Delta X_{23}$ must not occur before the surface of equal frequency $X=-\Delta X_{23}$ masks totally the stellar core. If this were not the case, the frequency intervals $X \in\left[X_{\min }, 0\right]$ and $Y \in\left[X_{\min }, 0\right]$ - i.e., $X \in\left[X_{\min }-\Delta X_{23},-\Delta X_{23}\right]$ - in which the gradual occultations of the stellar core by the surfaces of equal frequencies $X$ and $Y$ respectively take place, would overlap and this would of course prevent the formation of a double absorption component. With the definition (94) for the frequency $X_{\min }$, let us finally notice that the inequality (96) is equivalent to say that the separation $\Delta v_{23}$ of the upper atomic levels 2 and 3 must be greater than the initial velocity $v_{0}$ of expansion of the envelope.

Following a similar reasoning, we can now establish that both the contributions $E_{1}(X) / E_{c}$ and $E_{2}(X) / E_{c}$ to the emission profile will also be resolved into a double emission component if the conditions (95) and (96) are fulfilled (see Figures 29-32). Because the degree of excitation in the envelope decreases outwardly, we expect the two maxima to occur around the frequencies $X=X_{\min }$ and $Y=X_{\min }$ (i.e., $X=$ $\left.X_{\mathrm{min}}-\Delta X_{23}\right)$ for the profile $E_{1}(X) / E_{c}$ and around $X=0$ and $Y=0$ (i.e., $X=-\Delta X_{23}$ ) for the contribution $\left(E_{2}(X) / E_{c}\right.$.

From all the preceding results, we conclude that a resonance doublet profile $E(X) / E_{c}$ formed in an A.E envelope will be itself resolved into a double $\mathrm{P}$ Cygni profile if, and only if, the conditions (95) and (96) are fulfilled, and that the maxima of the two emission components will be located within the spectral intervals $X \in] X_{\min }, 0[$ and $X \in] X_{\min }-\Delta X_{23},-\Delta X_{23}[$.

In agreement with Hewitt and Noerdlinger (1974), we also notice from our results that the resonance doublet profiles formed in A.E envelopes display more abrupt transitions between the emission and absorption components as the value of the accelerating parameter $l$ becomes more negative. Indeed, when $l$ decreases, the spatial unfolding of the surfaces of equal frequencies $X$ and $Y$ gets more pronounced around the central frequencies $X=0$ and $Y=0$ (see Figures 27 and 28) and, consequently, the maxima of the profiles appear themselves sharper (see Figures 29-32).

Finally, because of the complex dependence of a resonance doublet profile formed 
in an A.E envelope on the nature of the velocity field $v(r)$, the distribution of the fictive opacities $\tau_{12}$ and $\tau_{13}$, the separation $\Delta v_{23}$ of the upper atomic levels, etc., we cannot propose a general method allowing the direct interpretation of an observed resonance doublet profile in terms of the physical and geometrical conditions prevailing in the envelope. However, we can always derive some useful information from the analysis of an observed resonance doublet profile: for instance, the maximal extent $v_{i}$ in velocity of the P Cygni absorption component towards the short wavelengths is related to the maximal expansion velocity $v_{\text {max }}$ by the relation

$$
v_{i}=-\left(v_{\max }+\Delta v_{23}\right)
$$

and, consequently, the knowledge of the separation $\Delta v_{23}$ of the upper atomic levels allows us to determine the quantities $v_{\max }$ and $\Delta X_{23}$. From these results, it is then also possible to calibrate the observed profile as a function of the dimensionless frequency $X \in\left[-1-\Delta X_{23}, 1\right]$ (see relation (88)). Furthermore, depending on whether the observed profile is resolved or not into a double P Cygni profile, we can set restrictions (see condition (95)) on the nature of the distribution of the fictive opacity $\tau_{12}$ in the medium. We would finally have to solve Equation (92) for various velocity and opacity distributions until an optimal fit with the observations was achieved. Whether a good agreement can be reached between the theory and the observations depends, of course, on the validity of the proposed model.

\section{Formation of Resonance Doublet Profiles in D.E Envelopes}

\section{(a) General equations}

Assuming that the source functions $S_{12}, S_{13}$ and the distributions of the fictive opacities $\tau_{12}, \tau_{13}$ have been determined throughout a D.E envelope (see Section 5) and adopting the same formalism as that developed in Section 6(A), we can derive straightforwardly the general expression $E(X) / E_{c}$ of a resonance doublet profile iormed in a D.E envelope.

First, let us consider the behaviour of the surfaces of equal frequencies $X$ and $Y=X+\Delta X_{23}$ from which line photons, emitted with a local frequency $v_{L} \simeq v_{12}$ and $v_{L}^{\prime} \simeq v_{13}$ along a direction parallel to the line of sight, contribute respectively to the monochromatic intensity functions $I_{12}(X)$ and $I_{13}\left(Y=X+\Delta X_{23}\right)$ entering expression (87) for the total amount of spectral energy $E(X)$. Adopting the velocity distributions (16) for $l=2$ and (27) for $G=10$, we have solved Equations (88) and (89) for different values of the frequency $X \in\left[-1-\Delta X_{23}, 1\right]$. The resulting 'surfaces of equal frequencies $X$ and $Y=X+\Delta X_{23}$, as seen by a fixed observer, are respectively illustrated with dashed and full lines in Figures $33(l=2)$ and $34(G=10)$ for the values of the parameters $\Delta X_{23}=0.25$ and $L_{\max }=5$. Also for the case of D.E envelopes, we have presented and studied in Paper IV the spatial unfolding of the 
surfaces of equal frequency $X$ as a function of $X \in[-1,1]$. In particular, we remind the reader that for a given value of the impact parameter $L p \in\left[0, L_{\max }\right]$, the direction parallel to the line of sight can intersect a surface of equal frequency $X$ in zero, one (single surface) or two distinct points (double surface). Finally, let us point out that except for a frequency retardation equal to $\Delta X_{23}$, the spatial unfolding of the surfaces of equal frequency $Y$ is identical to that of the surfaces of equal frequency $X \in[-1,1]$.

In ' general context, let us now suppose that for a given value of the impact parameter $L p \in\left[0, L_{\max }\right]$, the direction parallel to the line of sight intersects the surfaces of equal frequencies $X$ and $Y=X+\Delta X_{23}$, respectively, at two distinct points $R_{1}, R_{2}$, and $R_{1}^{\prime}, R_{2}^{\prime}$ (see Figure 33) and that these points lie, for instance, nearest to the observer in the order $R_{2}^{\prime}, R_{2}, R_{1}, R_{1}^{\prime}$. We can then easily deduce (cf. relation (90)) the expressions for the intensities $I_{12}(X)$ and $I_{13}(Y)$ appearing in

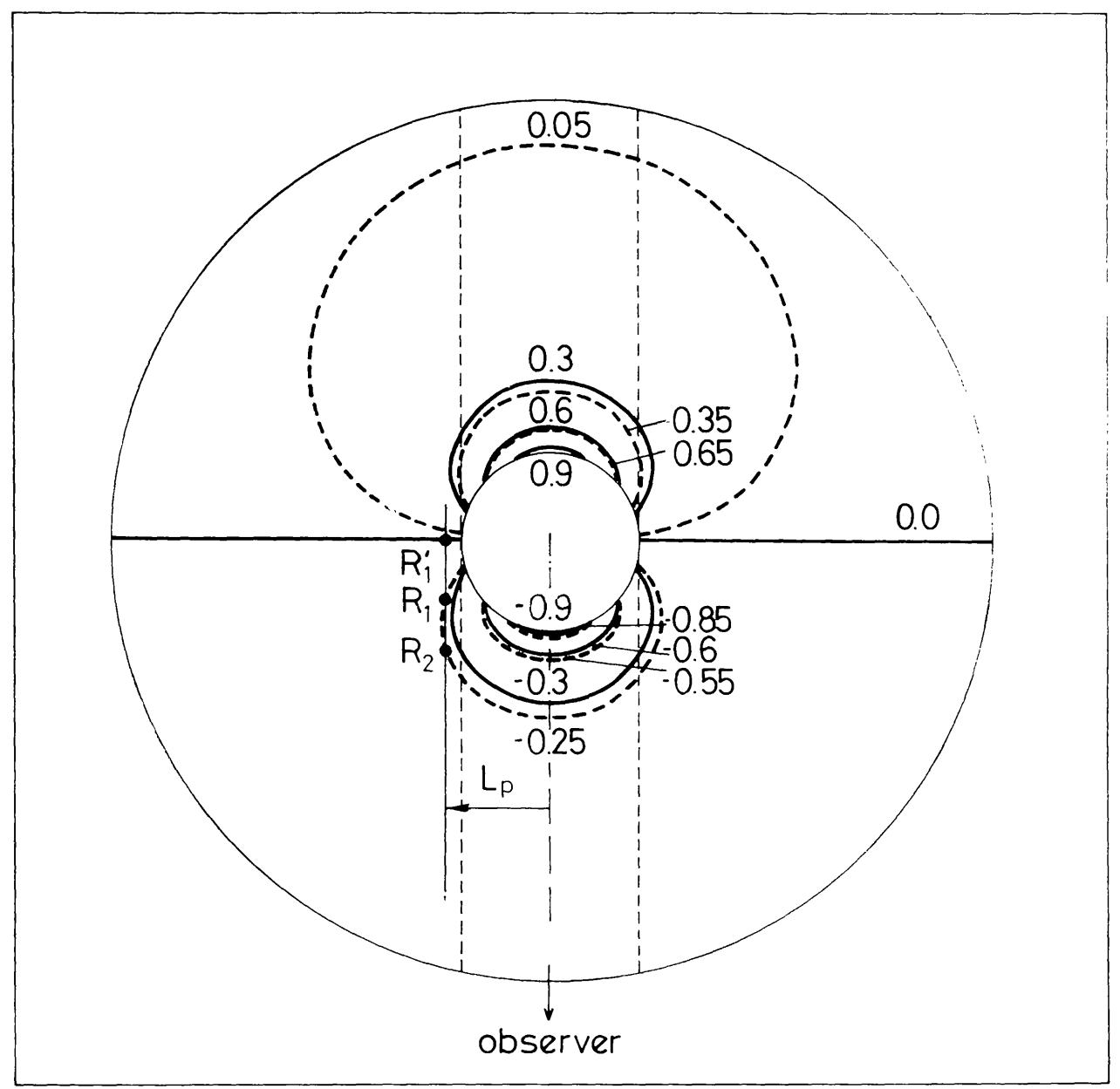

Fig. 33. Surfaces of equal frequencies $X=-0.85,-0.55,-0.25,0.05,0.35,0.65$ and $Y=X+\Delta X_{23}$ in a D.E envelope for the values of the parameters $l=2, \Delta X_{23}=0.25$ and $L_{\max }=5$. 


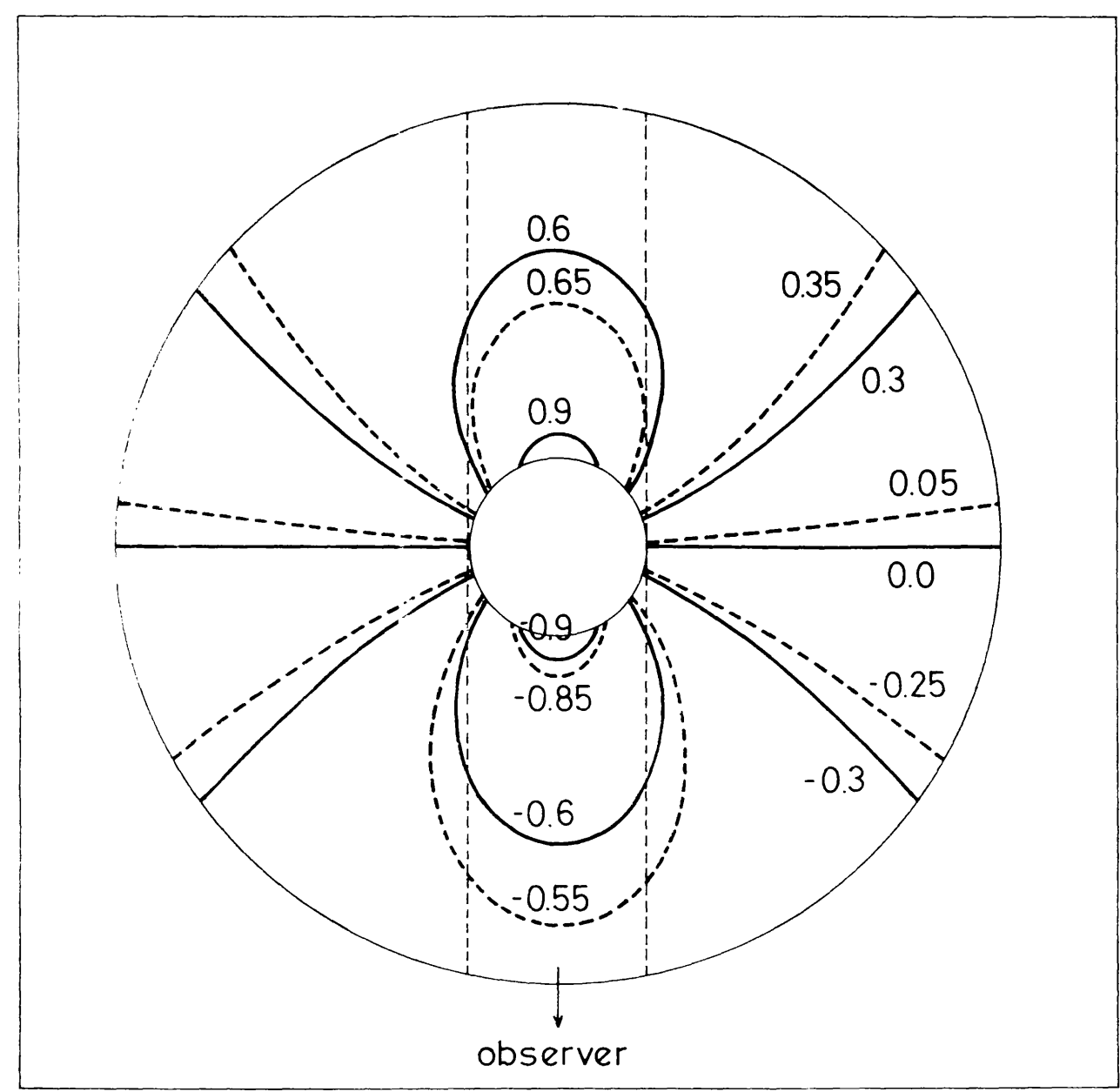

Fig. 34. Surfaces of equal frequencies $X=-0.85,-0.55,-0.25,0.05,0.35,0.65$ and $Y=X+\Delta X_{23}$ in a D.E envelope for the values of the parameters $G=10, \Delta X_{23}=0.25$ and $L_{\mathrm{max}}=5$.

relation (87). We directly find that

$$
\begin{aligned}
I_{12}(X)= & S_{12}\left(R_{1}\right)\left\{1-\exp \left[-\tau_{12}\left(R_{1}\right)\right]\right\} \exp \left[-\tau_{12}\left(R_{2}\right)\right] \exp \left[-\tau_{13}\left(R_{2}^{\prime}\right)\right]+ \\
& +S_{12}\left(R_{2}\right)\left\{1-\exp \left[-\tau_{12}\left(R_{2}\right)\right]\right\} \exp \left[-\tau_{13}\left(R_{2}^{\prime}\right)\right]
\end{aligned}
$$

and

$$
\begin{aligned}
I_{13}(Y)= & S_{13}\left(R_{1}^{\prime}\right)\left\{1-\exp \left[-\tau_{13}\left(R_{1}^{\prime}\right)\right]\right\} \exp \left[-\tau_{12}\left(R_{1}\right)\right] \exp \left[-\tau_{12}\left(R_{2}\right)\right] \times \\
& \times \exp \left[-\tau_{13}\left(R_{2}^{\prime}\right)\right]+S_{13}\left(R_{2}^{\prime}\right)\left\{1-\exp \left[-\tau_{13}\left(R_{2}^{\prime}\right)\right]\right\},
\end{aligned}
$$

with the implicit convention that if for some values of the frequency $X \in\left[-1-\Delta X_{23}, 1\right]$ and of the impact parameter $L p \in\left[0, L_{\text {max }}\right]$, Equations (88) and/or (89) have no solutions $R_{1}$ and/or $R_{2}, R_{1}^{\prime}$ and/or $R_{2}^{\prime}$, the fictious opacities $\tau_{12}\left(R_{1}\right)$ and/or $\tau_{12}\left(R_{2}\right), \tau_{13}\left(R_{1}^{\prime}\right)$ and/or $\tau_{13}\left(R_{2}^{\prime}\right)$ are set equal to zero. Furthermore, because the order of the intersection points can be different - in some cases the points $R_{2}, R_{2}^{\prime}, R_{1}^{\prime}, R_{1}$ lie, respectively the nearest to the observer - we easily allow for this fact by interchanging the indices 
12 by 13 , and vice versa, and the points $R_{2}^{\prime}, R_{2}, R_{1}, R_{1}^{\prime}$ by the points $R_{2}, R_{2}^{\prime}, R_{1}^{\prime}, R_{1}$ in relation (98).

Concerning the contribution to the resonance doublet profile $E(X) / E_{c}$ due to the radiation emitted by the stellar core, we similarly find (cf. relation (91)) that

$$
\begin{aligned}
I_{12}(X)+I_{13}(Y)= & I_{c} \exp \left[-\tau_{13}\left(R_{1}^{\prime}\right)\right] \exp \left[-\tau_{12}\left(R_{1}\right)\right] \times \\
& \times \exp \left[-\tau_{12}\left(R_{2}\right)\right] \exp \left[-\tau_{13}\left(R_{2}^{\prime}\right)\right]
\end{aligned}
$$

Finally, because of the symmetry of the envelope around the line of sight and with the help of relations (86), (87), (98) and (99), the expression of the resonance doublet profile formed in a D.E envelope takes the form

$$
\begin{aligned}
\frac{E(X)}{E_{c}}=2\left[\int _ { 0 } ^ { L _ { \operatorname { m a x } } } \left(\frac{S_{12}\left(R_{1}\right)}{I_{c}}\left\{1-\exp \left[-\tau_{12}\left(R_{1}\right)\right]\right\} \times\right.\right. \\
\quad \times \exp \left[-\tau_{12}\left(R_{2}\right)\right] \exp \left[-\tau_{13}\left(R_{2}^{\prime}\right)\right]+ \\
\quad+\frac{S_{12}\left(R_{2}\right)}{I_{c}}\left\{1-\exp \left[-\tau_{12}\left(R_{2}\right)\right]\right\} \exp \left[-\tau_{13}\left(R_{2}^{\prime}\right)\right]+ \\
\quad+\frac{S_{13}\left(R_{1}^{\prime}\right)}{I_{c}}\left\{1-\exp \left[-\tau_{13}\left(R_{1}^{\prime}\right)\right]\right\} \exp \left[-\tau_{12}\left(R_{1}\right)\right] \times \\
\quad \times \exp \left[-\tau_{12}\left(R_{2}\right)\right] \exp \left[-\tau_{13}\left(R_{2}^{\prime}\right)\right]+ \\
\left.\quad+\frac{S_{13}\left(R_{2}^{\prime}\right)}{I_{c}}\left\{1-\exp \left[-\tau_{13}\left(R_{2}^{\prime}\right)\right]\right\}\right) \operatorname{Lpd} \mathrm{d} p+ \\
+\int_{0}^{1} \quad \exp \left[-\tau_{13}\left(R_{1}^{\prime}\right)\right] \exp \left[-\tau_{12}\left(R_{1}\right)\right] \times \\
\left.\quad \times \exp \left[-\tau_{12}\left(R_{2}\right)\right] \exp \left[-\tau_{13}\left(R_{2}^{\prime}\right)\right] \operatorname{Lpd} L p\right]
\end{aligned}
$$

for $X \in\left[-1-\Delta X_{23}, 1\right]$.

(в) Numerical applications

Adopting the velocity distributions given in (16) and (27), we have solved rigorously Equation (100) for the resonance doublet profile $E(X) / E_{c}$ under different physical and geometrical conditions prevailing in D.E envelopes. For simplicity, we have assumed that the populations of the atomic levels are fully determined by the radiative excitations $(\varepsilon=0)$ due to the transfer of line photons in both resonance transitions $1 \rightleftharpoons 2$ and $1 \rightleftharpoons 3$ and that the equation of continuity (see relation (6)) describes the distribution of the density $n(L)$ of the atoms as a function of the distance $L$ to the centre of the stellar core.

The results of our calculations are illustrated in Figures 35-40. Each of these 
figures contains four graphs. The first graph displays the resulting profile $E(X) / E_{c}$ as a function of the frequency $X \in\left[-1-\Delta X_{23}, 1\right]$ and the three others give information about its individual contributions $E_{1}(X) / E_{c}, E_{2}(X) / E_{c}$ and $E_{3}(X) / E_{c}$ (see Section 6(в)).

Table IV summarizes the sets of parameters used for the calculations of each profile. In that table, the different columns give, respectively, the number of the figure and the relevant values of the parameters $l$ (or $G$ ), $\Delta X_{23}$ and $\tau_{12}^{l}$.

For all numerical applications, we fixed the maximal extension of the envelope to $L_{\max }=5$. As previously, we have chosen the oscillator strengths such that $f_{13}=$ $2 f_{12}$, and thus $\tau_{13}^{l}=2 \tau_{12}^{l}$, and the statistical weights of the atomic levels $g_{1}=2$, $g_{2}=2$ and $g_{3}=4$ in agreement with the atomic parameters of a typical resonance doublet $\left({ }^{2} S_{1 / 2}-{ }^{2} P_{1 / 2,3 / 2}^{0}\right)$.

\section{(c) Discussion of the Results}

First, we wish to confirm the fact that when the relative separation $\Delta X_{23}$ of the two upper atomic levels becomes negligibly small, the resonance doublet profiles calculated here are identical to the line profiles obtained directly by means of the two-level atom model (see Paper IV) for the values of the parameters $\tau_{12}^{l^{\prime}}=\tau_{12}^{l}+\tau_{13}^{l}$, $g_{1}^{\prime}=2$ and $g_{2}^{\prime}=6$. For the case of Figure $36\left(\Delta X_{23}=0.001\right)$ the calculated resonance doublet and single line profiles do not show variations between themselves exceeding $0.1 \%$.

The general appearance of resonance doublet profiles formed in D.E envelopes is striking (see Figures 35-40): they are characterized by an abrupt transition between the first, or unique, P Cygni absorption component and the first, or unique, emission line component, as well as by a sharp cut-off of the red wing of the emission line at the level of the stellar continuum. Furthermore, the violet wing of the second, or unique, P Cygni absorption component always reaches smoothly the level of the stellar continuum up to the extreme frequency $X_{i}=-1-\Delta X_{23}$, corresponding to the greatest velocity $v_{i}$ observed on the resonance doublet profile (cf. relation (97)). When the composite profile is resolved into a double P Cygni profile, we do similarly

TABLE IV

Caption for Figures $35-40$

\begin{tabular}{llll}
\hline Figure & $l$ (or $G)$ & $\Delta X_{23}$ & $\tau_{12}^{l}$ \\
\hline 35 & 0.5 & 0.50 & 1 \\
36 & 1 & $10^{-3}$ & 1 \\
37 & 1 & 0.25 & 1 \\
38 & 1 & 0.50 & 1 \\
39 & 2 & 0.50 & 1 \\
40 & $G=10$ & 0.25 & 1 \\
\hline
\end{tabular}



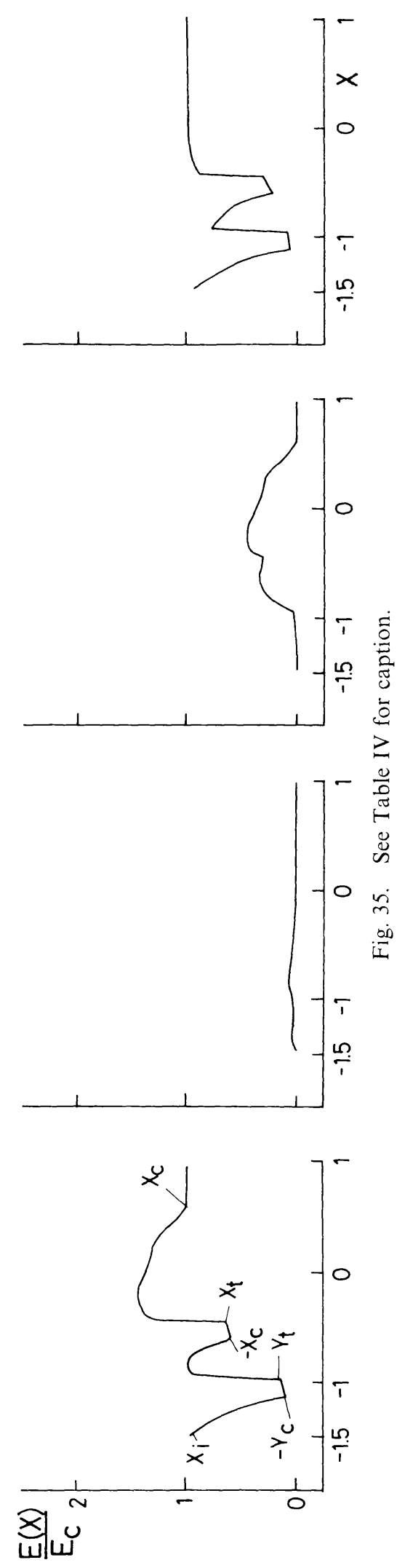
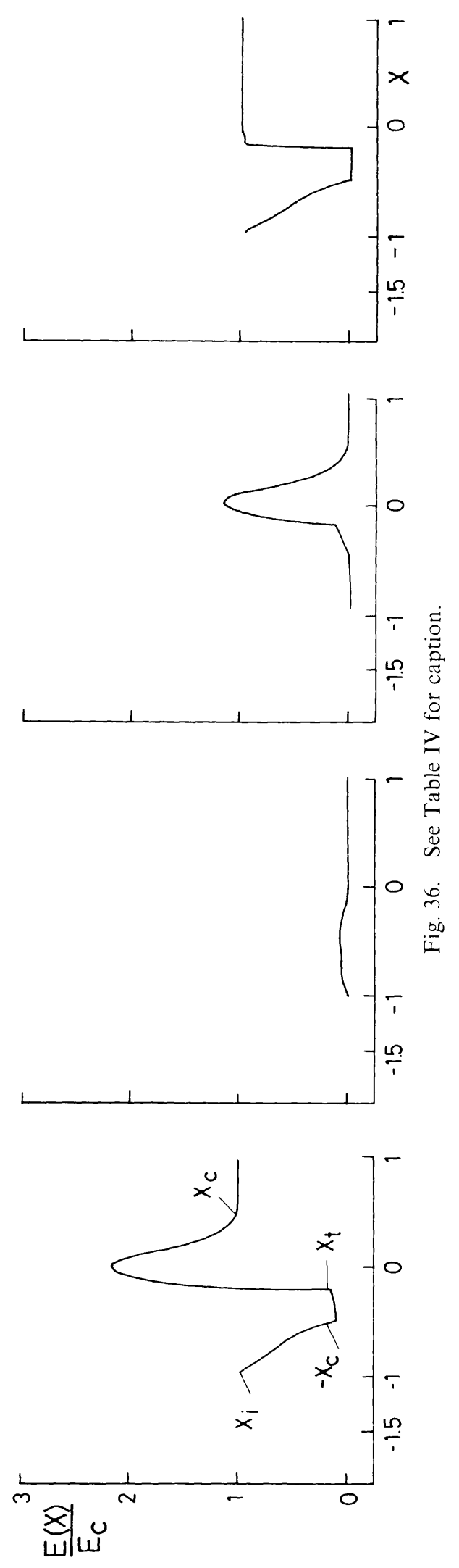

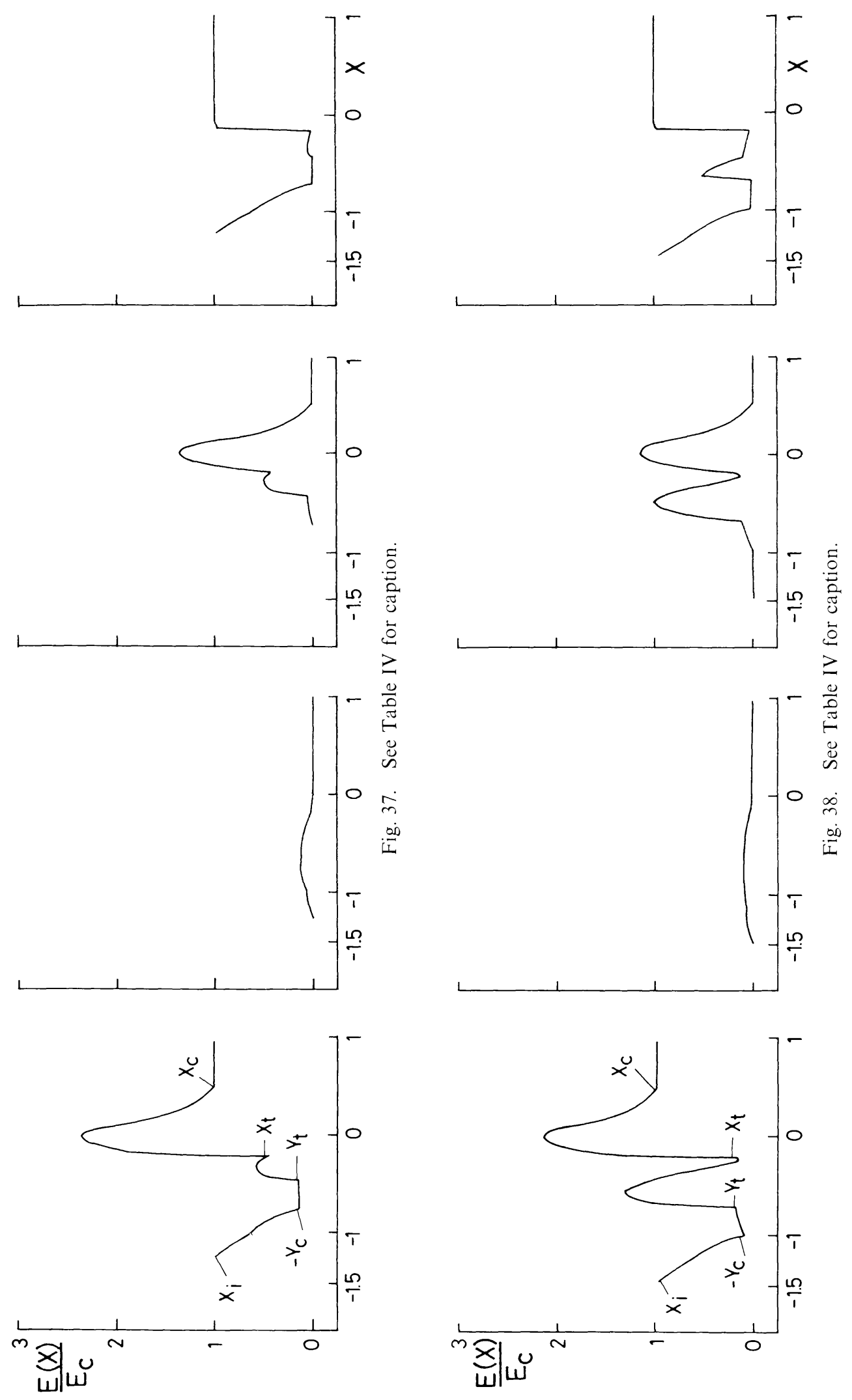

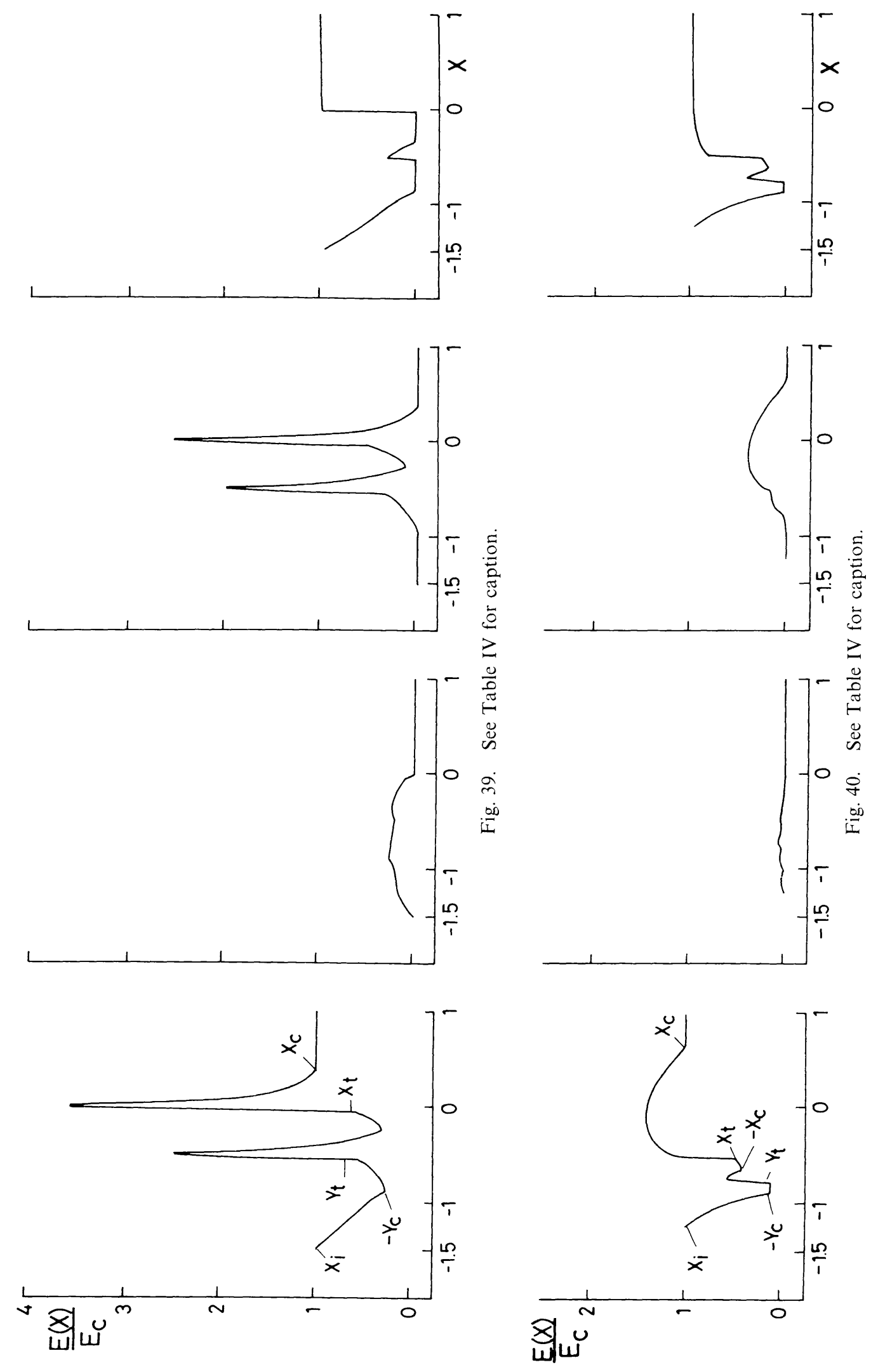
observe an abrupt transition between the second P Cygni absorption component and the second emission line component.

In order to account for these remarkable features, we discuss below the behaviour of the different contributions $E_{1}(X) / E_{c}, E_{2}(X) / E_{c}$ and $E_{3}(X) / E_{c}$ to the composite profile $E(X) / E_{c}$ as a function of the spatial unfolding of the surfaces of equal frequencies $X$ and $Y=X+\Delta X_{23}$ for $X \in\left[-1-\Delta X_{23}, 1\right]$.

For the velocity distributions (16) and (27), we shall first establish the values of the frequencies $X_{c}, X_{0}$ and $X_{t}$, associated with three particular surfaces of equal frequency $X$, defined as

(i) The frequency $X_{c}$ refers to the 'total occultation' frequency such that for $X \in\left[X_{c}, 1\right]$ the surfaces of equal frequency $X$ are totally occulted by the stellar core (see Figures 33 and 34). Imposing the condition that for the value of the impact parameter $L p=1$, the direction parallel to the line of sight is tangent to the surface of equal frequency $X_{c}$, we respectively find that

and

$$
X_{c}=[l /(l+1)]^{l / 2}(l+1)^{-1 / 2}
$$

with

$$
X_{c}=\sqrt{\left[1-\sin ^{2}\left(\theta_{a}\right)\right]\left[1+G \sin \left(\theta_{a}\right)\right] /(1+G)},
$$

$$
\sin \left(\theta_{a}\right)=-\frac{1}{3} G+\sqrt{\frac{1}{3}+\left(\frac{1}{3} G\right)^{2}},
$$

for the velocity distributions (16) and (27). Let us remark that the frequency $X_{c}$ only depends on the value of the decelerating parameter $l$ (or $G$ ) of the envelope.

(ii) The frequency $X_{0}$ represents the particular frequency at which the double surfaces of equal frequency $X$ (i.e., such as $|X|>X_{0}$ ) transform themselves into single surfaces (i.e., such as $|X|<X_{0}$ ). Noticing that, at a distance $L=$ $L_{\max }$ from the stellar core, the surface of equal frequency $X_{0}$ is tangent to the direction parallel to the line of sight, we find for the respective velocity distributions (16) and (27) that

and

$$
X_{0}=(l+1)^{-1 / 2} L_{\max }^{-l}
$$

$$
X_{0}=\left(1+\frac{G}{L_{\max }}\right) / \sqrt{(1+G)\left(1+\frac{3}{2} \frac{G}{L_{\max }}\right)} .
$$

(iii) Finally, $X_{t}$ refers to the greatest frequency value for which atoms, located along the corresponding surface of equal frequency, can still absorb the radiation emitted by the stellar core. With the condition that the line of sight intersects at one point the surface of equal frequency $X_{t}$ at a distance $L=L_{\max }$, we respectively obtain

and

$$
X_{t}=-L_{\max }^{-l}
$$

$$
X_{t}=-\left[\left(1+G / L_{\max }\right) /(1+G)\right]^{1 / 2},
$$

for the velocity distributions (16) and (27). 
Inspection of the fourth set of curves, illustrated in Figures 35-40, directly shows that the absorption profile $E_{3}(X) / E_{c}$ formed in a D.E envelope, for the case of a resonance doublet, appears resolved into two $\mathrm{P}$ Cygni absorption components whenever the condition

$$
\Delta X_{23}>X_{c}+X_{t}
$$

is fulfilled. Indeed, assuming that the relative separation $\Delta X_{23}$ of the upper atomic levels is appreciable, the first $\mathrm{P}$ Cygni absorption component results from the progressive occultation of the stellar disk by the surfaces of equal frequency $X \in\left[-1,-X_{c}\right]$. When $X>-X_{c}$, the surfaces of equal frequency $X$ then occult totally the stellar disk, and the residual intensity reaching a fixed observer is approximately given by the attenuation factor $\exp \left[-\tau_{12}(X, \theta=0)\right]$, where $\tau_{12}(X, \theta=0)$ represents the radial fictive opacity evaluated at the intersecting point between the line of sight and the corresponding surface of equal frequency $X$. When $X>X_{t}$, the surfaces of equal frequency $X$ cease occulting the stellar disk and therefore, at $X=X_{t}$, the absorption profile $E_{3}(X) / E_{c}$ reaches almost vertically the level of the stellar continuum. The spectral interval on whose sides the wings of the first P Cygni absorption component appear is thus given by $X \in\left[-X_{c}, X_{t}\right]$. We can similarly establish that the spectral interval on whose sides the wings of the second $\mathrm{P}$ Cygni absorption component are located is given by $Y \in\left[-X_{c}, X_{t}\right]$; i.e., $X \in\left[-X_{c}-\Delta X_{23}, X_{t}-\Delta X_{23}\right]$. Consequently, the absorption profile $E_{3}(X) / E_{c}$ will be resolved into two P Cygni absorption components if, and only if, the two spectral intervals, defined above, are not overlapping; i.e., if the condition

$$
X_{t}-\Delta X_{23}<-X_{c}
$$

equivalent to (104), is fulfilled. For the values of the parameters given in Table IV, we readily find that condition (104) is verified for the cases of Figure $35(l=0.5$, $\left.\Delta X_{23}>0.17\right)$, Figure $38\left(l=1, \Delta X_{23}>0.30\right)$, Figure $39\left(l=2, \Delta X_{23}>0.345\right)$ and Figure $40\left(G=10, \Delta X_{23}>0.122\right)$, in perfect agreement with the results of our numerical applications. We also understand that the absorption profile $E_{3}(X) / E_{c}$ illustrated in those figures reaches a maximum between the two $\mathrm{P}$ Cygni absorption components at a frequency $X \simeq X_{t}-\Delta X_{23}$ and that the residual intensities observed in the frequency intervals $X \in\left[-X_{c}, X_{t}\right]$ and $X \in\left[-X_{c}-\Delta X_{23}, X_{t}-\Delta X_{23}\right]$ are approximately given by the attenuation factors $\exp \left[-\tau_{12}(X, \theta=0)\right]$ and $\exp \left[-\tau_{13}(X, \theta=0)\right]$. When condition (104) is violated (see Figures 36 and 37), the spectral intervals $X \in\left[-X_{c}, X_{t}\right]$ and $X \in\left[-X_{c}-\Delta X_{23}, X_{t}-\Delta X_{23}\right]$ are overlapping and the absorption profile $E_{3}(X) / E_{c}$ is rigorously determined by the geometrical aspect of the surfaces of equal frequencies $X$ and $Y$ which occult, partially or totally, the stellar core as well as by the values of the attenuation factors $\exp \left[-\tau_{12}(X, \theta=0)\right]$ and $\exp \left[-\tau_{13}(Y, \theta=0)\right]$ evaluated along those surfaces (see Equation (100)).

The behaviour of the contribution $E_{2}(X) / E_{c}$ to the composite profile, illustrated 
by the third set of curves in Figures 35-40, can be qualitatively interpreted on the basis of the spatial unfolding of the surfaces of equal frequencies $X$ and $Y$ located in the regions outside the directions parallel to the line of sight for the value of the impact parameter $L p=1$ (see Figures 33 and 34). As for all numerical applications illustrated in Figures 30-35, the conditions $\tau_{12} \geqslant 1$ and $\tau_{13}>1$ (see relations (20), (30) and Table IV) are fulfilled across the media, it naturally follows that most of the contribution to the emission profile $E_{2}(X) / E_{c}$ is due to spectral line radiation emitted with a local frequency $v_{L} \simeq v_{12}$ and/or $v_{L}^{\prime} \simeq v_{13}$ from the regions of the double, or single, surfaces of equal frequencies $X$ and/or $Y$ located the nearest to the observer. So, in the spectral interval $Y \in\left[-X_{c}, X_{t}\right]$, i.e. $X \in\left[-X_{c}-\Delta X_{23}, X_{t}-\Delta X_{23}\right]$, most of the contribution to the emission profile $E_{2}(X) / E_{c}$ is due to spectral line radiation emitted with a local frequency $v_{L}^{\prime} \simeq v_{13}$ from the region of the double surface of equal frequency $Y$ located nearest to the observer. When the frequency $Y$ increases from $Y=-X_{c}$ to $Y=X_{t}$, the cross-section of that region, projected onto a plane perpendicular to the line of sight, constantly increases and therefore the emission profile $E_{2}(X) / E_{c}$ increases as well (see Figures 35-40). In the spectral interval $X \in\left[X_{t}-\Delta X_{23}, X_{t}\right]$, we have seen that the double surfaces of equal frequency $Y$ transform themselves into single surfaces and if the relative separation $\Delta X_{23}$ of the upper atomic levels is not too large, then the double surfaces of equal frequency $X$ occult progressively the former ones. The cross-section of the latter ones, projected onto a plane perpendicular to the line of sight, increasing as a function of the frequency $X \in\left[X_{t}-\Delta X_{23}, X_{t}\right]$, it follows that the emission profile $E_{2}(X) / E_{c}$ also increases in that same spectral interval (see Figures $35,36,40$ ). Let us note, however, that this reasoning holds as long as the emission of spectral line radiation from the unocculted part of the surfaces of equal frequency $Y$ is negligible. This situation obviously fails when the relative separation $\Delta X_{23}$ of the upper atomic levels becomes appreciable. For instance, if the condition

$$
\Delta X_{23}>X_{c}
$$

is fulfilled, the plane of equal frequency $Y=0$ is totally unocculted by the corresponding surface of equal frequency $X$. Indeed, by virtue of (105) we have $X<-X_{c}$. Consequently, the emission profile $E_{2}(X) / E_{c}$ will present a maximum at the frequency $Y=0$; i.e., $X=-\Delta X_{23}$. This is in good agreement with the results of our numerical applications illustrated in Figures $38\left(\Delta X_{23} \geqslant 0.5\right)$ and $39\left(\Delta X_{23} \geqslant 0.385\right)$. In reality, we can state that the plane of equal frequency $Y=0$ will not be appreciably occulted by the corresponding surface of equal frequency $X=-\Delta X_{23}$, as long as the latter remains a double surface. It will be so if the less restrictive condition

$$
\Delta X_{23}>\tilde{X}_{0}
$$

is satisfied, and in that case the emission profile $E_{2}(X) / E_{\mathrm{c}}$ will still present a distinct peak around the frequency $X=-\Delta X_{23}$. This result is in agreement with those of our numerical applications illustrated in Figures $35\left(l=0.5, \Delta X_{23}>0.37\right)$, Figures 
37 and $38\left(l=1, \Delta X_{23}>0.14\right)$, and Figure $39\left(l=2, \Delta X_{23}>0.02\right)$. For $X \in\left[X_{t}, X_{c}\right]$, the surfaces of equal frequency $Y=X+\Delta X_{23}$ are more and more occulted by the surfaces of equal frequency $X$. Consequently, if the medium is optically thick to the spectral line radiation in the resonance transition $1 \rightleftharpoons 2$, the emission profile $E_{2}(X) / E_{c}$ increases from $X_{t}$ towards greater frequencies and presents a maximum at $X=0$. For $X>0$, the surfaces of equal frequency $X$ change their curvature with respect to the plane of equal frequency $X=0$, and their cross-sections decreasing progressively as $X$ increases, so does the emission profile $E_{2}(X) / E_{c}$ which naturally vanishes for $X \geqslant X_{c}$. If the medium is not, strictly speaking, optically thick to the spectral line radiation in the resonance transition $1 \rightleftharpoons 2$ (cf. Figures 35 and $40, \tau_{12} \simeq 1$ ), the emission profile $E_{2}(X) / E_{c}$ reaches its maximum at a frequency $\left.X \in\right] X_{t}, 0[$ due to the fact that the surfaces of equal frequency $X$ are no longer totally opaque to the spectral line radiation emitted with a local frequency $v_{L}^{\prime} \simeq v_{13}$ from the surfaces of equal frequency $Y$. Recalling (see Section 5(D)) that for the values of the parameters $l>0.5$, $\tau_{12}^{l}=1$ and $\Delta X_{23}$ sufficiently large, the distribution of the source function $S_{12}$ tends towards the one directly calculated by means of the two-level atom model $\left(\tau_{12}^{\prime}>1\right)$, it is normal to notice that for $Y>X_{c}$ (i.e., $X \in\left[X_{c}-\Delta X_{23}, X\right]$ ), the contribution $E_{2}(X) / E_{c}$ to the composite profile illustrated in Figure $38\left(l=1, \Delta X_{23}=0.5\right)$ appears nearly identical to the one illustrated in Figure $36\left(l=1, \Delta X_{23}=0.001\right)$.

Concerning the contribution $E_{1}(X) / E_{c}$ to the composite profile, illustrated by the second set of curves in Figures 35-40, it is rigorously determined by the geometrical aspect of the surfaces of equal frequencies $X$ and $Y$ located inside the directions parallel to the line of sight for the value of the impact parameter $L p=1$ as well as by the distributions of the source functions $S_{12}, S_{13}$ and the fictive opacities $\tau_{12}$, $\tau_{13}$ evaluated along those surfaces. The emission profile $E_{1}(X) / E_{c}$ is evidently formed in the spectral interval $X \in\left[-1-\Delta X_{23}, 0[\right.$, and, while being much smaller than the absorption profile $E_{3}(X) / E_{c}$ for $X \in\left[-1-\Delta X_{23},-X_{c}-\Delta X_{23}[\right.$ or the emission profile $E_{2}(X) / E_{c}$ for $X \in\left[-X_{c}-\Delta X_{23}, 0[\right.$, the former does not show any welldefined structure which can be seen on the resulting profile $E(X) / E_{c}$.

Since the behaviour of the resulting profile $E(X) / E_{\mathrm{c}}$ in the frequency interval $X \in\left[-1-\Delta X_{23}, 1\right]$ is fully determined by those of its contributions $E_{1}(X) / E_{c}$, $E_{2}(X) / E_{c}$ and $E_{3}(X) / E_{c}$ mentioned above, we shall briefly summarize hereafter the characteristic features of resonance doublet profiles formed in D.E envelopes. This, in turn, will provide us with a method for interpreting an observed resonance doublet profile in terms of the physical and geometrical conditions prevailing in the D.E envelope.

(i) As the violet wing of the second, or unique, P Cygni absorption component always reaches smoothly the level of the stellar continuum up to the extreme frequency $X_{i}=-1-\Delta X_{23}$ (see Figures 35-40), corresponding to the greatest velocity $v_{i}$ observed on the resonance doublet profile, the knowledge of the separation $\Delta v_{23}$ (see relation (15)) of the upper atomic levels allows us to 
determine directly (see relation (97)) the maximal expansion velocity $v_{0}$ of the D.E envelope. From this result, it is then possible to derive the relative separation $\Delta X_{23}$ of the upper atomic levels (see relation (17)) and to calibrate the observed profile as a function of the dimensionless frequency $X_{c} \in\left[-1-\Delta X_{23}, 1\right]$ (see relation (88)).

(ii) We have stated before that the sharp cut-off of the red wing of the emission component at the level of the stellar continuum occurs at the total occultation frequency $X_{c}$ (see Figures 35-40). By means of relation (101), we can therefore deduce the value of the decelerating parameter $l($ or $G$ ) and thus, also the type of velocity field (see relations (16) and (27)) characterizing the D.E envelope.

(iii) The abrupt transition between the first, or unique, P Cygni absorption component and the first, or unique, emission line component taking place at the frequency $X_{t}$ (see Figures 35-40), the maximal extension $L_{\max }$ of the envelope is readily derived from relation (103).

(iv) If conditions (104) and/or (106) are fulfilled, the P Cygni absorption and/or emission components are resolved into two distinct components and it follows that the resonance doublet profile itself gets resolved into a double P Cygni one (see Figures 35, 37-40). We can generally locate on these profiles the frequencies $-X_{c}$ and $-Y_{c}=-\left(X_{c}+\Delta X_{23}\right)$, corresponding to characteristic features in the violet wings of the first and second P Cygni absorption components, as well as the frequency $Y_{t}=X_{t}-\Delta X_{23}$ at which takes place the abrupt transition between the second $\mathrm{P}$ Cygni absorption and emission components. Consequently, the location of the frequencies $-X_{c},-Y_{c}$ and $Y_{t}$ on an observed resonance doublet profile also allows one to infer the values of the parameters $l$ (or $G$ ) and $L_{\max }$.

In order to interpret an observed resonance doublet profile in terms of the physical and geometrical parameters of the envelope, we must finally solve Equation (100) for the composite profile $E(X) / E_{c}$ on the basis of the derived quantities $v_{0}, l$ (or $G$ ), $\Delta X_{23}$ and $L_{\max }$ for various choices of the distribution of the radial fictive opacity $\tau_{12}(L, \theta=0)$. Comparing the results of those numerical applications with the observed profile, we must adjust the function $\tau_{12}(L, \theta=0)$ until a good agreement is achieved between the theory and the observations. Such a confrontation has actually been performed with success for the observed resonance doublet profiles of C IV and Si IV in the spectra of the quasars PHL 5200 and RS 23 (Surdej and Swings, 1980).

\section{Conclusions}

Most of P Cygni profiles observed in the ultraviolet spectrum of early-type stars and of high redshift quasars are associated with resonance doublets for which the separation $\Delta v_{23}$ of the two upper atomic levels 2 and 3 is usually smaller than the maximal expansion velocity $v_{\max }$ of the envelope in which they are formed. In order to better 
understand the formation of such profiles, we have studied in this paper the transfer of spectral line radiation across spherically symmetric expanding envelopes for the case of a three-level atom model.

Using the integral form of the transfer equation in a frame moving with the fluid, we have established in the context of the Sobolev approximation the expressions for the different escape probabilities $\beta_{12}^{1}, \beta_{12}^{5}, \beta_{13}^{1}, \beta_{13}^{5}$, etc., and those for the various components of the spectral radiation fields $J_{12}$ and $J_{13}$ in order to study the behaviour of the source functions $S_{12}$ and $S_{13}$, of the radiative forces and the formation of resonance doublet profiles in A.E and D.E envelopes.

For the case of A.E envelopes, we have shown that the radiative interactions between atoms at a fixed point $R$ and the photons emitted with a local frequency $v_{L}^{\prime} \simeq v_{13}$ from the geometrical locus $\left(R, R^{\prime}\right)$ have the resulting effect to modify appreciably the population of the first upper atomic level with respect to its population directly obtained when neglecting those distant interactions.

We have also concluded that it is very important to take into account the structure of a resonance doublet when deriving the exact behaviour of the resulting radiative force $\mathbf{F}^{R E}$ acting on an atom in an A.E envelope. Indeed, in the regions near the stellar core where the medium is generally optically thick $\left(\tau_{12}>1\right)$, we have demonstrated that the resulting radiative force $\mathbf{F}^{R E}$ appears at least twice as high as the single radiative force $\mathbf{F}_{12}^{C A}$ calculated by assuming that the doublet constitutes a single line transition. In this context, we recall that Lucy and Solomon $(1967,1970)$ and Castor et al. (1975) have proposed an ejection mechanism, based on radiative pressure, in order to explain the origin of mass-loss observed in a great number of early-type stars. However, when evaluating the radiative force acting on the gas, these authors assumed that lines belonging to a same multiplet (resonance doublet, etc.) form a single line transition. As in most parts of the A.E envelopes they considered, the medium is optically thick to the spectral line radiation $\left(\tau_{12}>1\right)$; the fact that they did not include the structure of the multiplets in the calculation of the resulting radiative force $\mathbf{F}^{R E}$ implies that they underestimated the latter (at least in the regions near the star) and consequently also the rates of mass-loss they derived. Therefore, it would be essential to treat again the same hydrodynamical problem by including the structure of each multiplet.

We have illustrated and discussed the formation of resonance doublet profiles in A.E envelopes and we have shown that if the separation $\Delta v_{23}$ of the upper atomic levels is greater than the ejection velocity $v_{0}$ of the envelope and that if the medium is optically thin $\left(\tau_{12}<1\right)$ at distances $L>L\left(X=-\Delta \mathrm{X}_{23}\right)$ (see relation (95)), the composite profile $E(X) / E_{c}$ appears resolved into a double $\mathrm{P}$ Cygni profile and that the maxima of the emission line components are located around the central frequencies $X=0$ and $Y=0$ (i.e., $X=-\Delta X_{23}$ ), corresponding to the unshifted positions of the red and violet components of the resonance doublet.

For the case of D.E envelopes, we have illustrated numerically the behaviour of the source functions $S_{12}$ and $S_{13}$ under a variety of physical and geometrical condi- 
tions. We have concluded that the additional radiative interactions between the photons emitted with a local frequency $v_{L}^{\prime} \simeq v_{13}$ (resp. $\left.v_{L}^{\prime \prime} \simeq v_{12}\right)$ from the geometrical locus $\left(R, R^{\prime}\right)$ (resp. $\left.\left(R, R^{\prime \prime}\right)\right)$ and the atoms situated at a fixed point $R$ have the resulting effect of increasing (resp. decreasing) the population of the first (resp. second) upper atomic level, with respect to their populations derived when only considering the radiative interactions between the photons emitted with a local frequency $v_{L}^{\prime \prime \prime} \simeq v_{12}$ (resp. $\left.v_{L}^{\prime \prime \prime} \simeq v_{13}\right)$ from the geometrical locus $\left(R, R^{\prime \prime \prime}\right)$ and the atoms situated at the fixed point $R$.

We have also shown that in a D.E envelope, besides the extreme cases $\Delta X_{23} \ll 1$ or $\tau_{12}<1, \tau_{13}<1$ for which $\mathbf{F}^{R E} \simeq \mathbf{F}_{12}^{D E}$, the resulting radiative force $\mathbf{F}^{R E}$ acting on an atom, via the resonance transitions $1 \rightleftharpoons 2$ and $1 \rightleftharpoons 3$, is generally about two times greater than the force $\mathbf{F}_{12}^{D E}$ directly calculated by assuming that the resonance doublet forms a single line transition.

Under various physical and geometrical conditions prevailing in D.E envelopes, we have solved the general equation $E(X) / E_{c}$ of the resonance doublet profile and we have found that all calculated profiles are characterized by an abrupt transition between the first, or unique, P Cygni absorption and emission components as well as by a sharp cut-off of the red wing of the emission line at the level of the stellar continuum. We have then shown that if the separation $\Delta v_{23}$ of the upper atomic levels is sufficiently large (see conditions (104) and (106)), the composite profile $E(X) / E_{c}$ always appears resolved into a double P Cygni profile and that an abrupt transition also takes place between the second P Cygni absorption and emission components. On the basis of these remarkable features, we have investigated the possibility of interpreting an observed profile in terms of the physical and geometrical parameters of the envelope. We have concluded that if the observed profile is effectively formed in a D.E envelope, its analysis always allows to derive the maximal expansion velocity $v_{0}$ of the medium, the value of the decelerating parameter $l$ (or $G$ ) as well as the maximal extent $L_{\max }$ of the envelope in which it is formed. In order to determine the distribution of the density $n(L)$ of the atoms, and thus a rate of mass-loss, we have seen that we had to solve the general equation (100) of the profile for different choices of the distribution of the radial fictive opacity $\tau_{12}(L, 0=0)$ until a good agreement is achieved between the theory and the observations.

We should also mention that the formalism developed in this work can be easily generalized to the study of the transfer of spectral line radiation for other types of resonance multiplets: for instance, we shall mention the resonance doublets of $\mathrm{N}$ II ( $\lambda \lambda$ 1083.990, 1085.701 $\AA$ ), S IV ( $\lambda \lambda 1062.672,1073.045 \AA$ ), etc., characterized by two line transitions $1 \rightleftharpoons 3,2 \rightleftharpoons 3$ issued from two nearby lower atomic levels $(1,2)$ towards a common upper one (3).

Let us finally note that all the results (degree of excitation, radiative forces, profiles) derived here for the cases of A.E envelopes and D.E envelopes are directly and respectively applicable to the cases of inward-decelerated (I.D) and inward-accelerated (I.A) envelopes. 


\section{Acknowledgement}

I am very much indebted to Jean-Pierre Swings for discussion, advice and reading of the manuscript.

\section{References}

Appenzeller, I.: 1979, Astron. Astrophys. 71, 305.

Bertout, C.: 1977, Astron. Astrophys. 58, 153.

Castor, J. I.: 1970, Monthly Notices Roy. Astron. Soc. 149, 111.

Castor, J. I.: 1974, Monthly Notices Roy. Astron. Soc. 169, 279.

Castor, J. I., Abbott, D. C. and Klein, R. I.: 1975, Astrophys. J. 195, 157.

Castor, J. I. and Lamers, H. J. G. L. M.: 1979, Astrophys. J. Suppl. 39, 481.

Hewitt, T. G. and Noerdlinger, P. D.: 1974, Astrophys. J. 188, 315.

Ivanov, V. V.: 1973, in D. G. Hummer (ed.), N.S.B.-Spec. Publication 385.

Lucy, L. B.: 1971, Astrophys. J. 163, 95.

Lucy, L. B. and Solomon, P. M.: 1967, Astron. J. 72, 310.

Lucy, L. B. and Solomon, P. M.: 1970, Astrophys. J. 159, 879.

Magnan, C.: 1973, Ph.D. Dissertation, University of Paris (in French).

Mihalas. D.: 1970, Stellar Atmospheres, W. H. Freeman \& Co., San Francisco, p. 356.

Mihalas, D., Kunasz, P. B. and Hummer, D. G.: 1976, Astrophys. J. 210, 419.

Mundt, R. and Wolf, B.: 1977, IAU Coll., No. 42, p. 88.

Sobolev, V. V.: 1947, Dvizusciesja obolocki zvezd, Leningrad (transl. Moving Envelopes of Stars; translated from Russian by S. Gaposchkin, Harvard University Press, Cambridge, Mass., 1960).

Sobolev, V. V.: 1958, in V. A. Ambartsumyan (ed.), Theoretical Astrophysics, Chap. 28, Pergamon Press Ltd, London.

Surdej, J.: 1977, Astron. Astrophys. 60, 303 (Paper I).

Surdej, J.: 1978a, Astron. Astrophys. 66, 45 (Paper II).

Surdej, J.: 1978b, Astron. Astrophys. 62, 135 (Paper III).

Surdej, J.: 1979a, Astron. Astrophys. 73, 1 (Paper IV).

Surdej, J.: 1979b, Ph.D. Dissertation, University of Liège (Paper V; in French).

Surdej, J. and Swings, J. P.: 1980, Astron. Astrophys., in press.

Walker, M. F.: 1977, IAU Coll., No. 42, p. 92.

Wolf, B., Appenzeller, I. and Bertout, C.: 1977, Astron. Astrophys. 58, 163. 